2013-03-18

\title{
Online Survey System for Image-Based Clinical Guideline Studies Using the Delphi Method
}

Todd Martin Harper

Brigham Young University - Provo

Follow this and additional works at: https://scholarsarchive.byu.edu/etd

Part of the Computer Sciences Commons, and the Health Information Technology Commons

\section{BYU ScholarsArchive Citation}

Harper, Todd Martin, "Online Survey System for Image-Based Clinical Guideline Studies Using the Delphi Method" (2013). Theses and Dissertations. 3476.

https://scholarsarchive.byu.edu/etd/3476

This Thesis is brought to you for free and open access by BYU ScholarsArchive. It has been accepted for inclusion in Theses and Dissertations by an authorized administrator of BYU ScholarsArchive. For more information, please contact scholarsarchive@byu.edu, ellen_amatangelo@byu.edu. 
Online Survey System for Image-Based Clinical

Guideline Studies Using the Delphi Method

Todd Harper

A thesis submitted to the faculty of

Brigham Young University

in partial fulfillment of the requirements for the degree of

Master of Science

Chia-Chi Teng, Chair

Dale C. Rowe

Joseph J. Ekstrom

School of Technology

Brigham Young University

March 2013

Copyright (C) 2013 Todd Harper

All Rights Reserved 


\author{
ABSTRACT \\ Online Survey System for Image-Based Clinical \\ Guideline Studies Using the Delphi Method \\ Todd Harper \\ School of Technology, BYU \\ Master of Science
}

The increasing use of health information technology (HIT) is due to a rising interest in improving the quality of health care. HIT has the potential to reduce cost and transform services. Proper clinical support systems will contribute to the meaningful use of HIT systems by providing a wide array of data to clinicians for the diagnosis and treatments. Clinical guidelines, created by a consensus of experts, can be put in place to assist physicians in making clinical decisions. Delphi methods are commonly used to create consensus from surveys completed by a team of experts. Image-based studies could create guidelines that standardize severity, deformity or other clinical classifications. As these studies were traditionally conducted using paper-based media, the cost and time requirement often make the process impractical. Using state of the art Web 2.0 technologies, a web-based system can aid medical researchers in conducting imagebased Delphi studies for improved clinical guidelines and decision support.

Keywords: Todd Harper, Delphi, image ranking, web, clinical guidelines 


\section{ACKNOWLEDGEMENTS}

I am grateful for the support and encouragement that I have received during the completion of this thesis. I would like to thank my graduate chair, Dr. Chia-Chi Teng, for his guidance, not only on this project but throughout my academic career. I would like to thank my graduate committee, Dr. Dale Rowe and Dr. Joseph Ekstrom, for their contributions in time.

Finally, I am forever grateful to my wife, Amanda, for her endless support, encouragement, and faith in my abilities throughout this entire process. 


\section{TABLE OF CONTENTS}

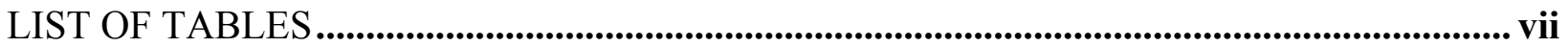

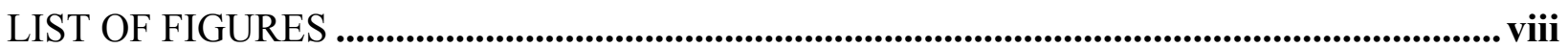

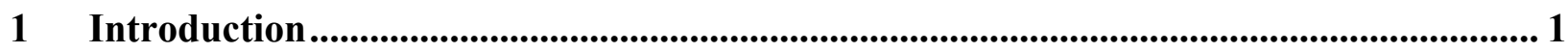

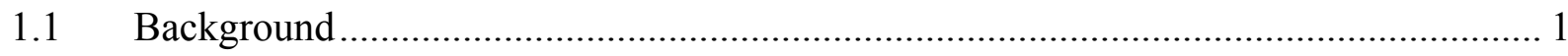

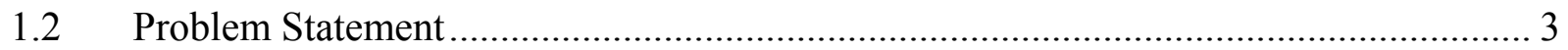

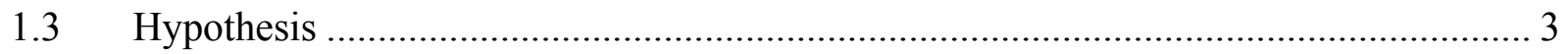

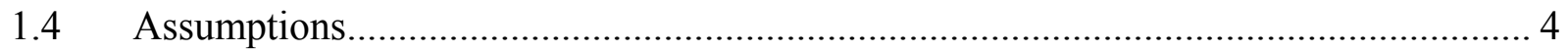

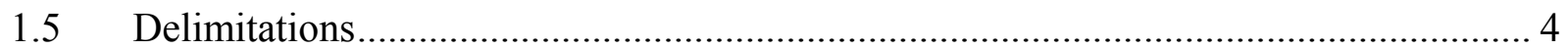

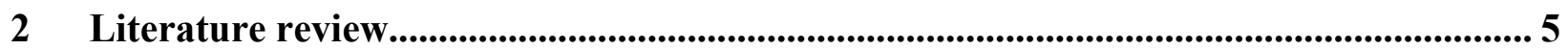

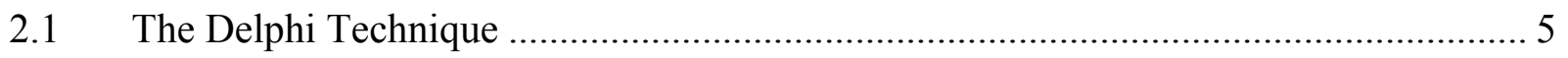

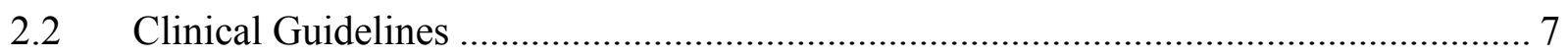

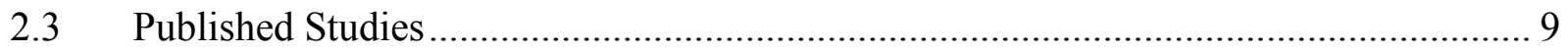

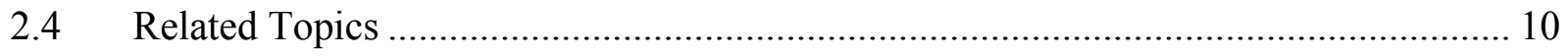

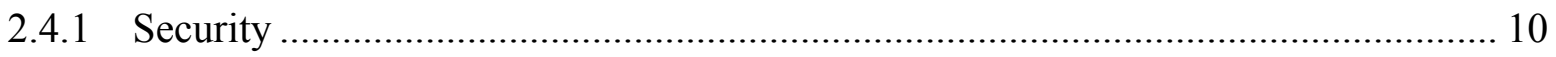

3 Implementation ..................................................................................................................... 12

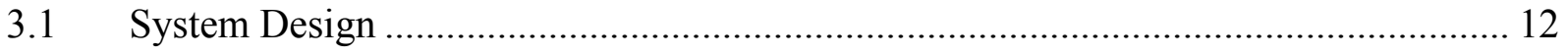

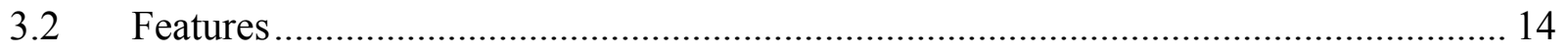

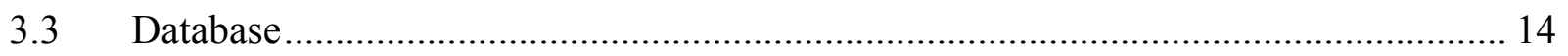

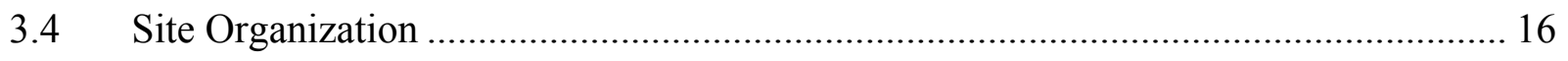

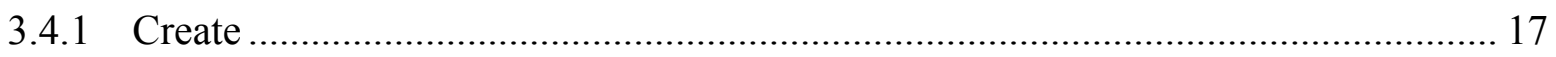

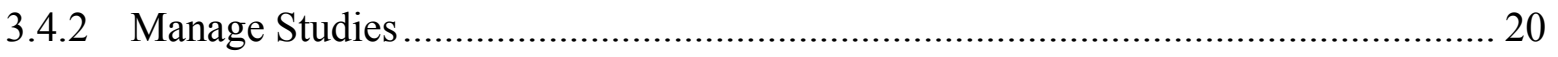

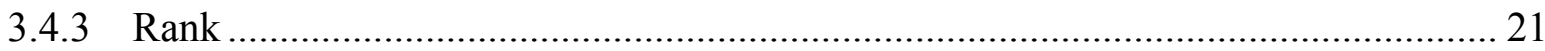

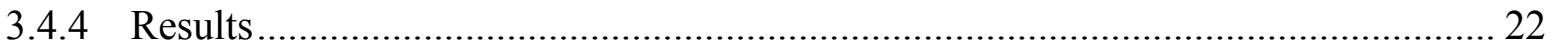

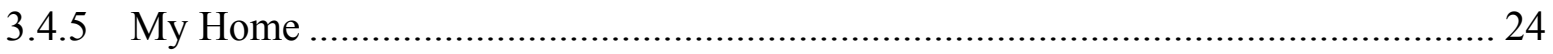




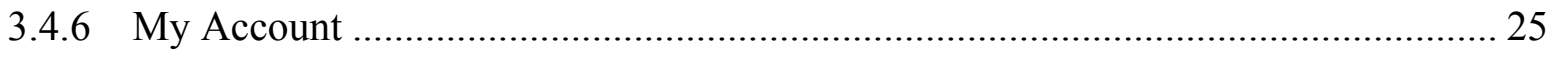

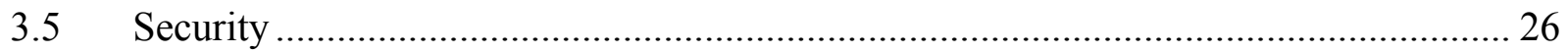

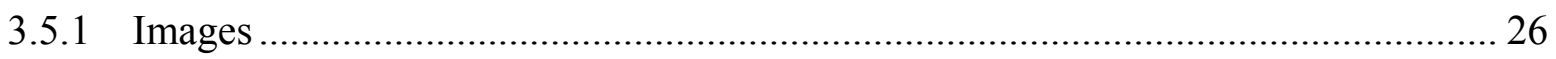

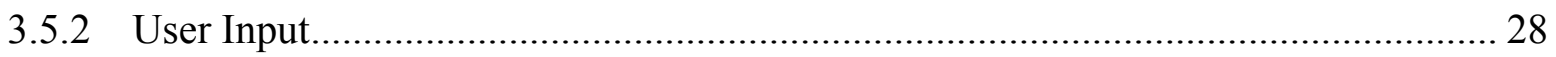

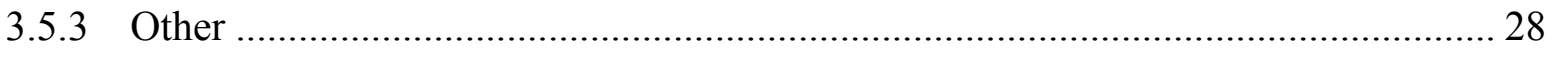

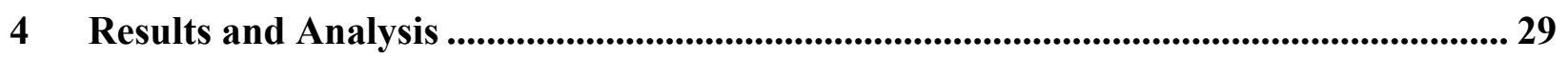

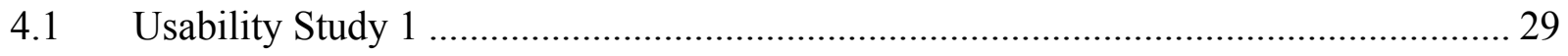

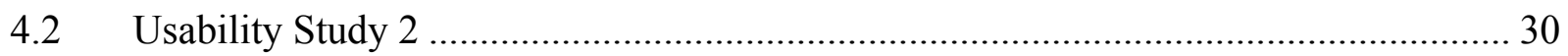

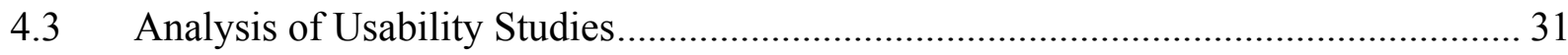

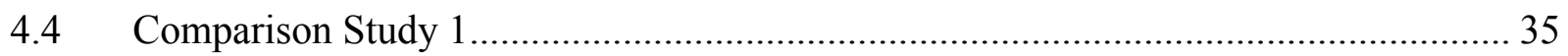

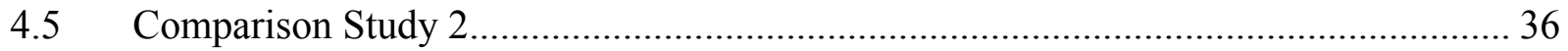

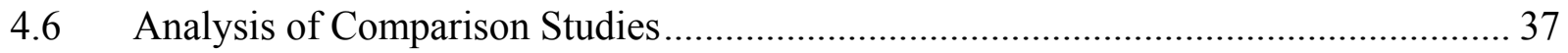

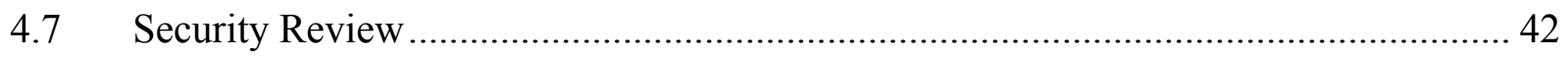

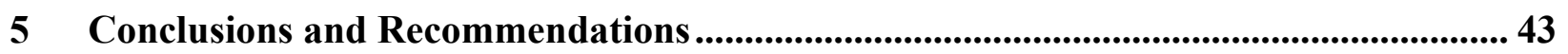

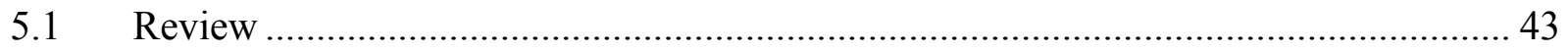

5.2 Recommendations for Action and Study ............................................................... 43

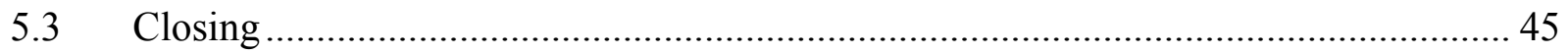

REFERENCES............................................................................................................................. 46

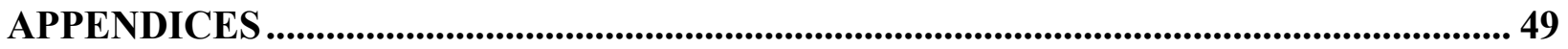

Appendix A. Penetration Test Report.............................................................................................. 50

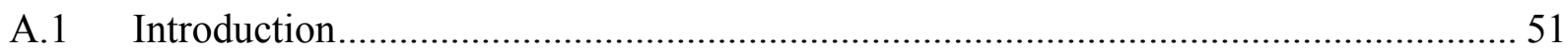

A.1.1 Executive Summary …………………………………………………………. 51

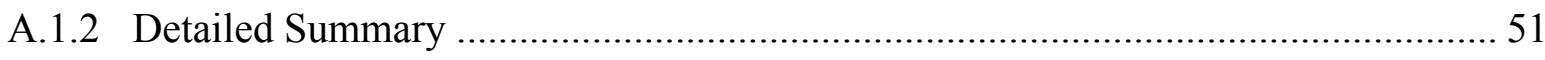

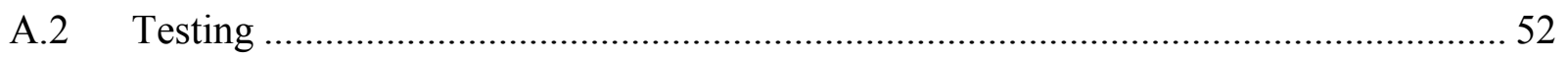

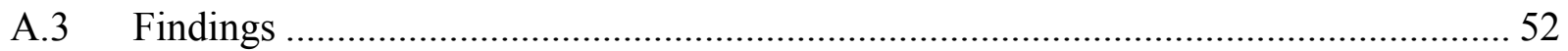




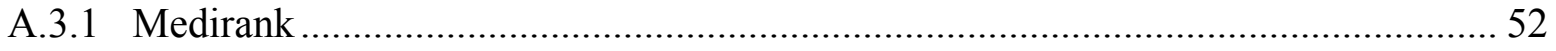

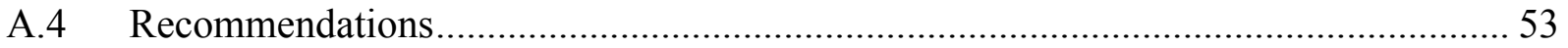

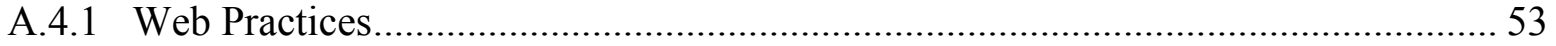

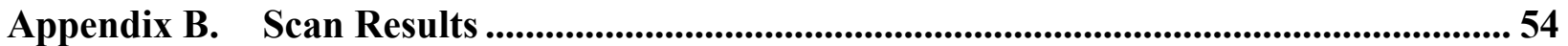

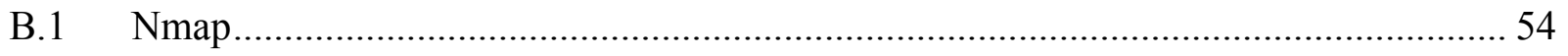

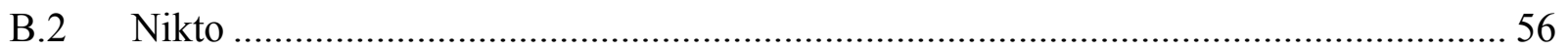

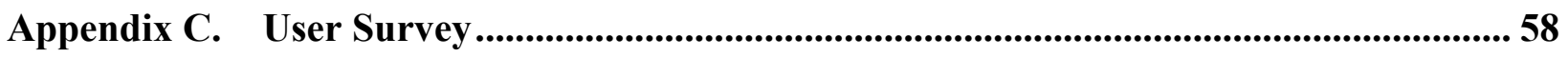

Appendix D. Site Code..........................................................................................................6 66

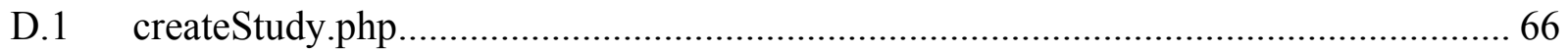

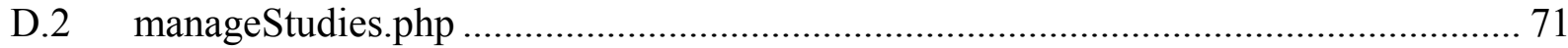

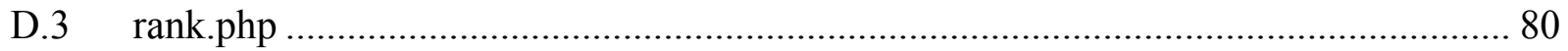

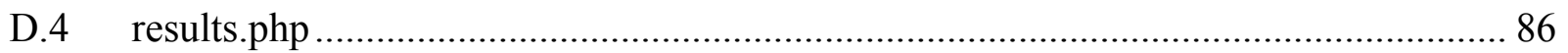

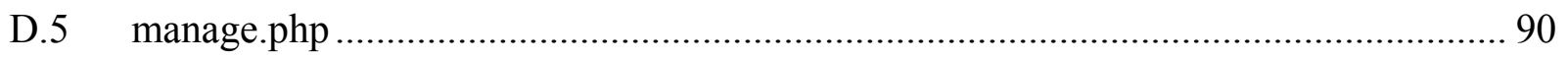

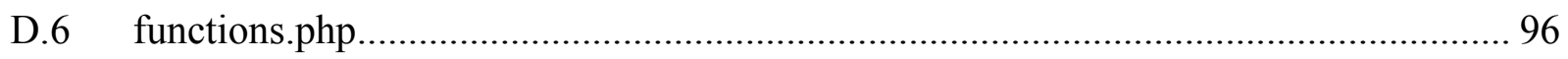




\section{LIST OF TABLES}

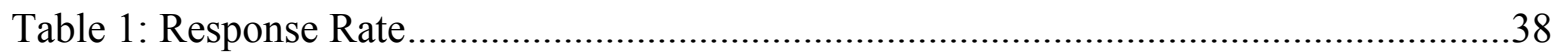

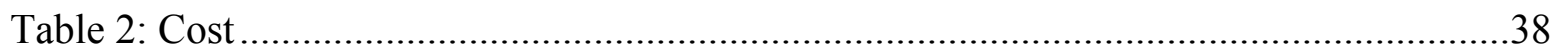

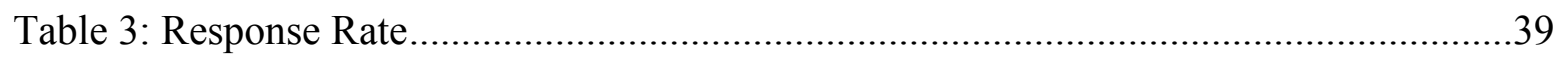

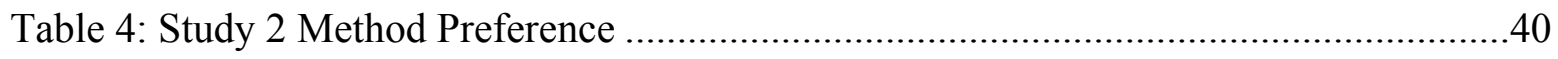

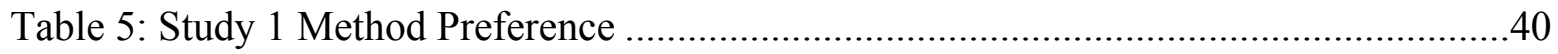

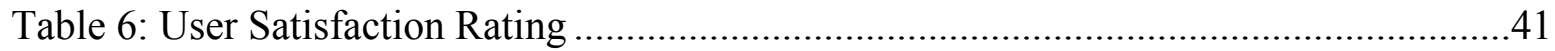

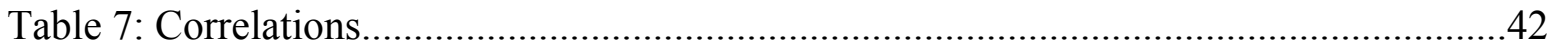




\section{LIST OF FIGURES}

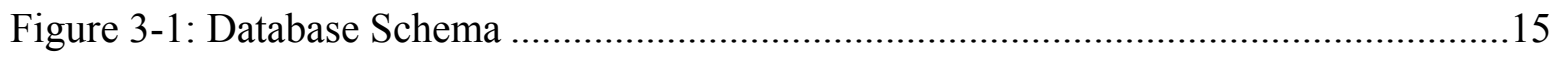

Figure 3-2: Medirank Site Organization ........................................................................ 17

Figure 3-3: Adding Expert in System ............................................................................ 18

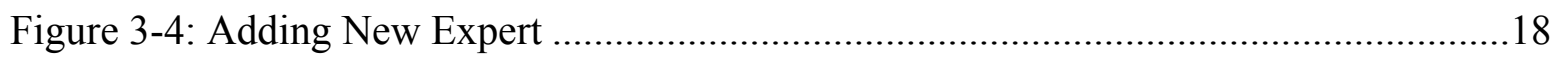

Figure 3-5: Uploading Images ................................................................................. 19

Figure 3-6: Workflow for Creating a Study.............................................................. 19

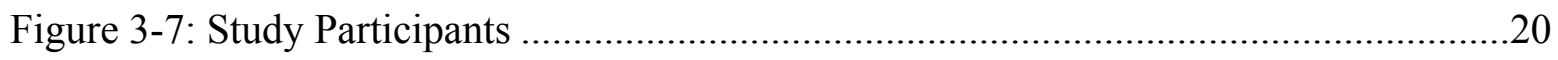

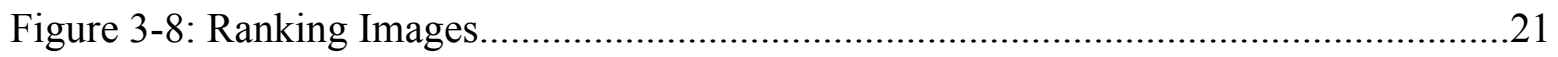

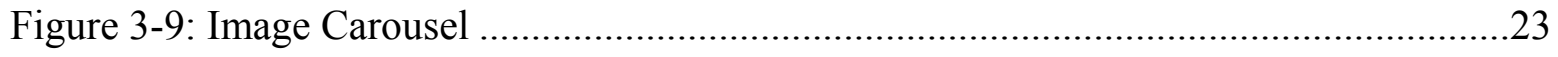

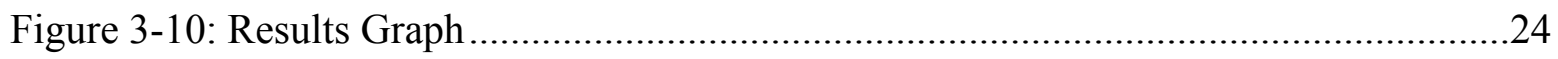

Figure 3-11: Table View of Full Data...........................................................................25

Figure 3-12: Landing Page Tables Showing Associated Studies ......................................26

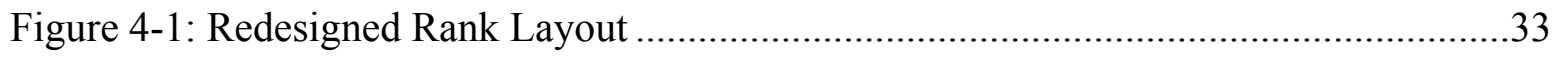

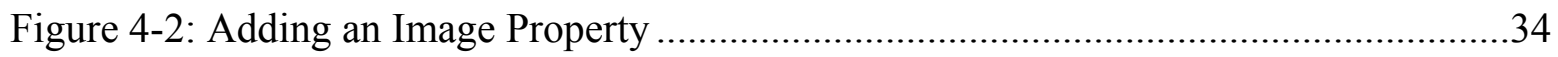

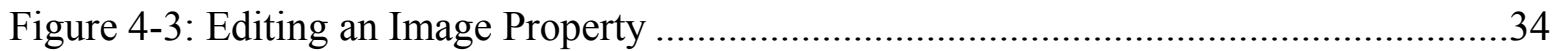




\section{INTRODUCTION}

\subsection{Background}

Information Technology (IT) is an emerging discipline with evolving research areas, educational boundaries and industrial applications. Despite its relative nascence, other disciplines, such as businesses, healthcare or government, are beginning to recognize the benefits of leveraging IT, such as computing systems that provide instant data transfer, storage of vast amounts of records, and worldwide collaboration.

The use of health information technology (HIT) has increased over the last decade and promises to transform services with improved quality and reduced cost (Blumenthal and Glasser 2007). Projects, both publicly and privately funded, are aiming to speed up the adoption and meaningful use of HIT as well as to protect privacy, enhance security and improve interoperability. Clinicians, consumers, politicians and insurers all share a common interest in improving the quality of healthcare (Field and Lohr 1992; Woolf, et al. 1999).

One particular area of improvement that is in the interest of both consumers and physicians is clinical guideline and decision support system. Subjective clinical decisions are made daily by caregivers based on personal experiences. In many of these cases, a standard of care is not defined. Therefore, it becomes the duty of the caregiver to decide what is best. Because of this subjectivity, patients with similar problems receive different care depending on location, clinician, or facility (Woolf, et al. 1999). To make objective clinical decisions, 
healthcare providers need access to accurate data and pertinent knowledge such as standards of care and treatment for each medical issue. To meet this need, healthcare providers are beginning to implement clinical decision support systems to aid in the daily assessments made by clinicians.

An important role of the clinical decision support system is to improve the quality of care received by patients by creating clinical guidelines. Field and Lohr state that: "These guidelines are systematically developed to assist practitioners and patient decisions about appropriate health care for specific clinical circumstances" (Field and Lohr 1990). The statements are intended to be a collection of current evidence and opinion of best practice. Ideally, the development of guidelines is based on a thorough evaluation of medical evidence and can include published studies on the outcomes of various treatments (National Health and Medical Research Council 1998). However, some diagnosis and treatment is still too subjective. In order for standards to be created objectively, an expert panel is needed to evaluate and create guidelines.

The Delphi Technique is commonly used in situations where individual judgments must be expressed in order to address the gaps in current knowledge (Powell 2002). A traditional Delphi study is comprised of multiple iterations of surveys or questionnaires, where feedback is collected and statements are modified, which allow the expert opinions to form a consensus.

In the past, Delphi studies were paper-based; but, printing, sending, and compiling the study and its results cost time and money. Basically, paper-based studies seem outdated and perhaps have become inadequate for meeting today's demands since web-based technologies can be used to create efficient and cost effective Delphi studies.

Most survey studies are text-based, meaning written statements are presented to participants of the study and a Likert scale is used to gauge agreement. However, for research 
dealing with standardization of deformity, severity or other clinical classifications, image-based studies are necessary. Fisher, et al. states that often in clinical practice "accurate subjective grading of an individual patient's deformity is more difficult because of the absence of direct comparison" (Fisher, Tse and Marcus 2008). By making image-based survey studies available, researchers may provide clinicians an opportunity to make a direct comparison between their patient in the clinic, and a standardized scale of deformity or severity.

\subsection{Problem Statement}

Clinicians should have the most updated and accurate information available to them when addressing cases of deformity. Unfortunately, paper-based studies do not fully serve this need due in part to the length of time required to conduct a study. Web technologies offer a medium that addresses the inefficiencies such as cost, time and distance of paper-based methods. This thesis details a web-based system intended for the use of health care researchers conducting image-based Delphi studies.

\subsection{Hypothesis}

A web-based image Delphi study performs better than a paper-based study as measured in the following areas:

H1. Time to task completion

H2. Cost to the researcher

H3. Response rate

H4. Method Preference

H5. User satisfaction 


\subsection{Assumptions}

This study assumes that researchers will have the approval of the Institutional Review Board of their respective institutions. It is also assumed that the researchers will create a final rank based on the results of the study which can be used to create clinical guidelines as appropriate.

\subsection{Delimitations}

This research details the design, creation and testing of an online survey system for image-based clinical guideline studies using the Delphi method. It neither attempts to rate the quality of potential studies, nor attempts to create clinical guidelines from the results of the studies. However, when the site is completed a usability study will be conducted to show functionality, and a comparison study will be done to test the hypothesis. Finally, a security review will be conducted to assess the level of security provided. 


\section{LITERATURE REVIEW}

\subsection{The Delphi Technique}

The Delphi technique was first developed by the Rand Corporation in the 1950s for technological forecasting (Goodman 1987; Hasson, Keeney and McKenna 2000). The traditional technique comprises multiple iterations of surveys or questionnaires broken up by controlled feedback (Linestone and Turoff 1975). Other characteristics of the Delphi technique are anonymity, statistical group response and expert input (Goodman 1987). This technique is particularly useful for situations where individual judgments must be expressed and combined to address an incomplete base of knowledge (Delbecq, Van de Ven and Gustafson 1975).

Consequently, it has been used in many areas of research, as illustrated by Lindeman in her pioneering use of the Delphi technique to determine clinical nursing research priorities (Lindeman 1975).

The Delphi technique is flexible in the way it is conducted. Due to this, many forms of the technique exist. For instance, McKenna (McKenna 1994) details a 'modified Delphi', Crisp et al. (Crisp, et al. 1997) describe a 'policy Delphi' and Beretta (Beretta 1996) discusses the “real-time Delphi." Because of the varied interpretations of the Delphi technique, Mead and Moseley proposed the term Delphi approach as a more accurate description of these studies (Mead and Moseley 2001). Regardless of the variances, the key principles are the same: poll a panel of experts to generate a consensus regarding a particular subject matter. 
The group structure of the Delphi technique gives it an advantage over many informal methods of reaching consensus. Other methods, such as committees, can be dominated by powerful individuals, exacerbated by the fact that only one individual can speak at a time (Murphy, et al. 1998). Rowe et al. describe this as a "process loss", meaning that the presence and actions of some in-group situations may stifle creativity and possibly result in ambiguous or even conflicting results. In contrast, they suggest that the democratic nature and participant anonymity in the Delphi format leads to a "process gain", because the situation cultivates collaboration and creativity (Rowe, Wright and Bolger 1991). However, some state that the consensus approach may lead to watered-down, bland statements and lack of accountability (Sackman 1975; Rennie 1981).

Obviously, the quality and quantity of expert participants is an important consideration when creating a panel because, as Powell states, the "success of a Delphi study clearly rests on the combined expertise of the participants who make up the expert panel" (Powell 2003). Powell emphasizes that panel size and expert qualifications are two key aspects of a successful expert panel. Powell asserts that experts should be specifically chosen based on their work in the area and credibility. She also explains that "[e]xperts in the clinical field may include expert clinicians, researchers with scientific expertise and patients/lay people who have expertise by virtue of having experienced the impact of a condition or intervention" (Powell 2003). Powell concludes that to create a representative panel, expert qualifications seem more important than sheer number of participants (Powell 2003). On the other hand, Murphy et al. believe that the more experts who participate the better although they concede that there is little empirical evidence to show the effect of panel size on the reliability and validity of the results (Murphy, et 
al. 1998). It is clear that the subject matter of the study must be taken into consideration when determining the size and quality of a representative expert panel.

This analysis of literature clearly shows that the Delphi approach is a popular and reliable technique for generating consensus because it can be customized to fill the needs of various disciplines and their degrees of specialization. This is especially beneficial to the health profession because the resulting consensuses can be used to form clinical guidelines.

\subsection{Clinical Guidelines}

Clinicians often make difficult choices concerning patient treatment. Because of uncertainty about the value of treatment options, practices can vary widely (Murphy, et al. 1998). Clinical guidelines are critical tools for improving practices because, as Field and Lohr state guidelines are "systematically developed statements to assist practitioner and patient decisions about appropriate health care for specific clinical circumstances" (Field and Lohr 1992). Ideally, guidelines would be based strictly on empirical evidence produced through rigorous testing. In practice however, few areas of healthcare have sufficient research-based evidence for guideline creation (Murphy, et al. 1998). For this reason, creating clinical guidelines based on expert consensus is necessary.

The use of clinical guidelines has increased since the early 1990 s due to a variety of factors. Woolf et al. cite rising healthcare costs, new technologies, variation in service delivery and quality, and an intrinsic desire of healthcare professionals as some reasons for the increased interest in clinical guidelines (Woolf, et al. 1999). Indeed clinicians, patients, politicians and insurers alike all have a vested interested in improving healthcare. 
Clinical guidelines are positive and beneficial to many parties; specifically patients, healthcare professionals, and researchers. These guidelines can promote effective treatment and discourage ineffective care to reduce morbidity and mortality as well as improve quality of life (Woolf, et al. 1999). For patients, the greatest benefit lies in their improved health outcomes. Another benefit for patients is consumer versions of the guidelines in the form of online repositories, such as webmd.com and mayoclinic.com. When patients are self informed, they can take a proactive approach to their healthcare and be empowered to make educated choices about care (Woolf, et al. 1999). For healthcare professionals, clinical guidelines can improve the quality of clinical decisions. For a clinician unfamilliar with a particular area, guidelines offer explicit recommendations about how to proceed. For researchers, guidelines highlight gaps in evidence and knowledge. Healthcare systems and insureres benefit from guidelines by receiveing a justification for a procedure (Woolf, et al. 1999).

While clinical guidelines provide great benefits, care must be taken to avoid the potential harms. In other words, guidelines and recommendations could be wrong. Flawed guidelines can institutionalize ineffective, harmful or wasteful care and the very same parties that stand to benefit from guidelines could be harmed. Also, incorrect guidelines can adversly affect insurance policies for patients. When used, these recommendations could lead insurance providers to drop access to care. Flawed guidelines also harm clinicians on a professional level because they promote inaccurate knowledge and compromise the quality of care provided. This, in turn, halts true scientific progress (Murphy, et al. 1998). To ensure quality guidelines, the process for development should identify treatment that provides the best possible health outcome (National Health and Medical Research Council 1998). 


\subsection{Published Studies}

Because determining severity is often subjective to the clinician, many studies have focused on quantifying the severity of various clinical classifications. For example, Qing et al. devised a functional MRI model to diagnose depression severity for female patients (Qing, et al. 2010). Gralnek et al. developed a scoring index for small bowel mucosal inflammatory change (Gralnek, et al. 2008). Schulman et al. created a scoring system for severity of hemophilia (Schulman, et al. 2008). In even another example, Thomas et al. created a mathematical model used in conjunction with CT scans to judge the severity of bone fractures (Thomas, et al. 2008). Each of these examples demonstrates a method to quantify severity using mathematical models. However, not all clinical classifications can be quantified mathematically and therefore a consensus of expert opinions is needed to bridge the gap in knowledge.

As previously stated, the Delphi technique has become a popular method of generating consensus in a variety of fields. In the medical field, Lindeman is perhaps the pioneer user of the Delphi method of research (Powell 2003). In this early study, she expounded on clinical nursing research priorities by polling nearly 400 nurses through four rounds (Lindeman 1975). Due to her success, other researchers began using the Delphi technique to converge expertise into guidelines for clinical use. One such study, carried out by Van der Linde and colleagues, focused on developing clinical guidelines for lower-limb prostheses prescription through a text-based approach. They state that "[d]eveloping guidelines is seen as one of the most potentially useful tools for achieving changes in behavior and, therefore, more uniform, high-quality care" (Van der Linde, et al. 2005). Using this motivation, these researchers conducted an internet-based Delphi study to create clinical guidelines for the justification and care of patients. The study, which polled 32 experts, was conducted over two rounds and a final consensus meeting, where a 
consensus was defined as "a general agreement of a substantial majority," meaning greater than 75 percent of experts agreed. It resulted in the generation of 37 postulates for prescription (Van der Linde, et al. 2005). This research is a good example of a text-based approach of the Delphi technique to generate clinical guidelines.

Not all guidelines can be successfully created through text; in some cases, images are necessary. Fisher, Tse and Marcus conducted an image-based Delphi study grading the severity of cleft lips. In this study, the researchers hypothesized that experts were able to reliably rank patients according to their deformity consistent with a set of anthropometric measurements. They took standard pre-surgical photographs and sent them to eight cleft surgeons. To assess agreement between the panelists, the interclass correlation coefficient was used. Their results show that the expert ranking of severity is consistent with the anthropometric measurements (Fisher, Tse and Marcus 2008).

In searching the literature for image-based Delphi studies, only the Fisher study was found. One possible reason for the lack of literature is the difficulty in carrying out these studies. Images must be printed off with sufficient quality that the experts can make an informed ranking. Once the study is complete, transferring the compiled data to a digital form for thorough analysis can cause errors in the data. An internet-based Delphi study engine focusing on image-based studies can simplify the research process and allow more studies of this nature to be made.

\subsection{Related Topics}

\subsubsection{Security}

The Health Insurance Portability and Accountability Act (HIPPA) of 1996 prescribe guidelines of privacy and security for entities involved in healthcare. Although this system is not 
a covered entity, care should be taken to protect those involved with this project because of the sensitive nature of medical research. To protect the researchers, those in the studies, the experts and the administrators of the survey system security measures must be in place.

Image files contain more data than the image itself, for example, the Exchangeable image file format (Exif), created by the Japan Electronic Industry Development Association (JEIDA), specifies the formats commonly used by digital cameras for images and sound (ISO 12234-1). This format allows for data about GEOlocation, date and time, equipment, image author, and more to be attached as metadata to the image file. It is possible that this data could be used to identify patients in the study or healthcare facilities. There are tools available which can remove this data and neutralize the potential threat.

Cross-site scripting (XSS) and SQL injection are other threats to mitigate. XSS is a class of vulnerabilities that allow injection of code into the client side of a web application. The code, when executed, sends information about the user back to the attacker. Vogt et al. state that the "optimal approach to prevent XSS attacks would be to eliminate the vulnerabilities in the affected web applications. To this end a web application must properly validate all input, and in particular remove malicious scripts" (Vogt, et al. 2007). In a similar vein, SQL injection is a vulnerability that allows an attacker to execute queries on a database and return results. To avoid this risk, steps similar to those for XSS mitigation should be taken. 


\section{IMPLEMENTATION}

As discussed in chapter 2, though there is flexibility in the way Delphi studies are conducted, there are a few fundamental elements: anonymity, expert panels, and results, such as usable data, to form conclusions. This research details a web-based system that meets these basic requirements.

\subsection{System Design}

In order to meet the fundamental elements of a Delphi study, a platform with very specific characteristics is required. These characteristics include:

Scalability: It is hoped that with time, this system will become increasingly well-known and used within the medical research community. As more studies are created, images uploaded, users added and studies completed, the database and file system will grow. As the database continues to grow, features such as search, creation, and result compilation must scale to remain efficient.

Longevity and Stability: This tool is not meant to be temporary or a one-time use. In order for the system to be effective, multiple researchers need to consistently use it and the data must stay accessible. Therefore, the system needs to be continuously available for years to come. Any significant downtime could deter other researchers from using this tool. The system also needs to be compatible with systems in the future. 
Database Modeling: The survey system is designed to add experts to a study based on a primary or secondary specialty. These same experts should be allowed to participate in multiple studies at a time or author their own studies. Study authors are able to assign properties to each image in order to gather extra data.

Cross Platform: The intent of this system is to reach as many users as possible. As a result, the user base is broad, bringing with it a variety of base operating systems and browsers. Any use of proprietary and closed-source technology on the client, such as Microsoft Silverlight or Adobe Flash, could preclude some users from using the tool.

Inexpensive: In order for the site to be efficient, maintenance should be cost effective. Researchers will not be charged for the use of the tool. Though currently, there is no budget to support future maintenance, grants from the National Science Foundation or the National Institutes of Standards and Technology could be pursued. These grants could help pay for the undergraduate and graduate students who would perform system maintenance.

To address each of these characteristics, a platform was designed using PHP, JavaScript and jQueryUI and will be powered by a MYSQL database. Together these tools provide the necessary functionality. JavaScript and jQueryUI are supported across all browsers and desktop operating systems. A MYSQL database can scale proportionally by using a foreign key indexing feature necessary for database normalization. PHP is an open source server side language that seamlessly integrates with JavaScript, jQueryUI, MYSQL and HTML, as well as contains a rich feature set to interact with visiting users. 


\subsection{Features}

The entire basis for this project is to create a tool for Delphi studies focusing on image ranking. Certain features are critical for the successful creation and completion of these studies. The following are some of the features integrated into the site:

- Multiple image uploading

- Addition of experts

- Confidential distribution to participants

- Drag and drop of images for ranking

- Ability to save ranking progress

- Retrieval of full result data set

- Calculated correlation data

While these features meet the requirements for image ranking, they also address the fundamental elements of a Delphi study. More specifically, anonymity is met through the confidential distribution of surveys to the participants. The participating experts are not informed who the other participating experts are, nor can they view the study results until their own ranking has been submitted. The need for expert panels is addressed through the ability to customize the participant group. Finally, results used to form conclusions are provided through retrievable data sets and calculated correlations.

\subsection{Database}

As noted in section 3.2, the site is powered by a MYSQL backend database. The database is designed to provide a stable backbone for all the desired features as well as minimize data replication. Figure 3-1 shows the entire schema diagram for the database. 
As is shown in the below figure, the database is designed around three core tables: users, study, and images. The other surrounding tables are supporting tables for the core three.

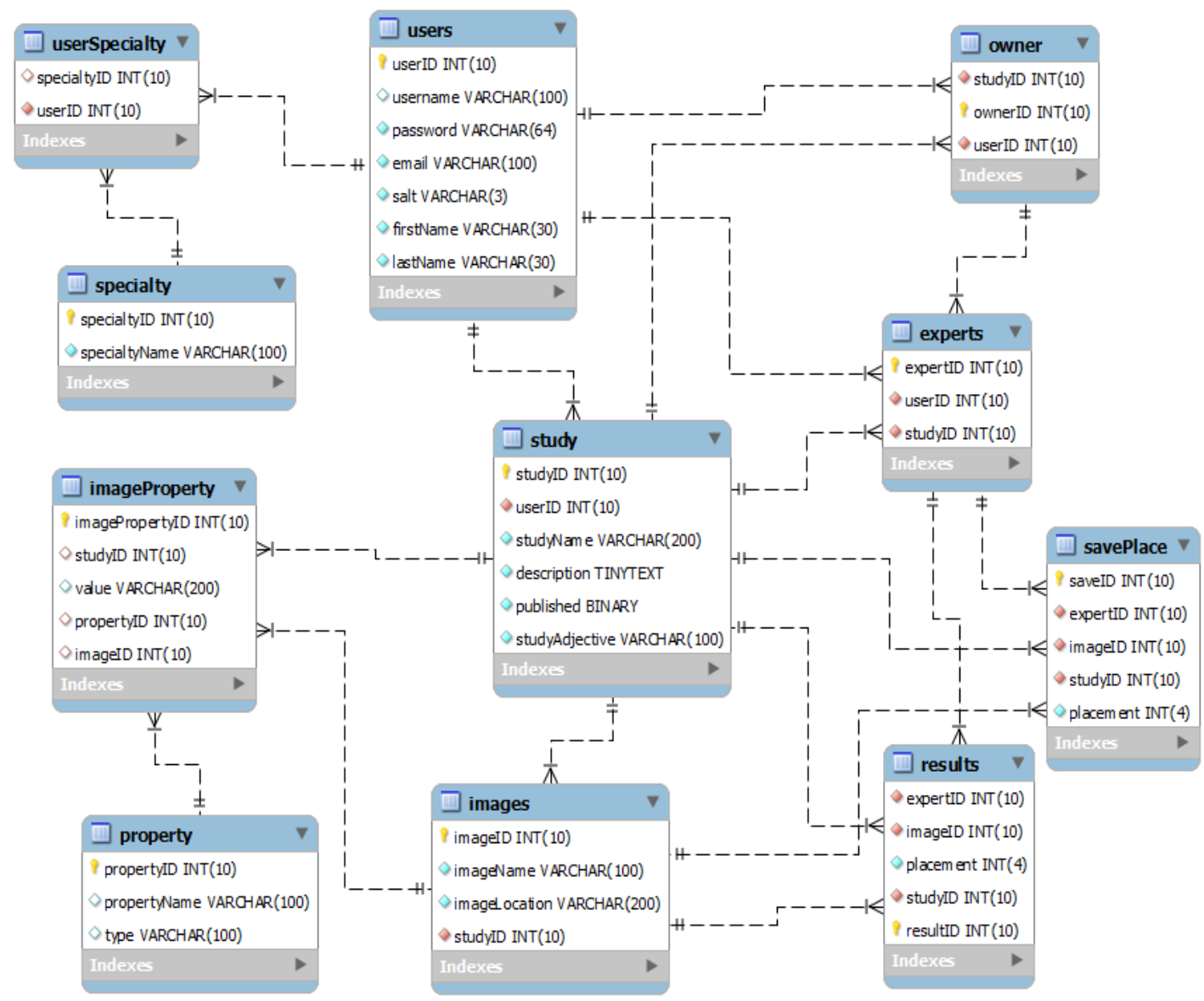

Figure 3-1: Database Schema

The users table contains relevant information about the users such as email, username, password and salt. The owner and expert tables both refer back to the users table, since a user can be an owner and an expert at the same time. A user can pick which specialties that interest them, and a study owner can leverage that information by selecting users who have an interest in the specialty corresponding to their respective study. 
The study table contains information pertaining to the study, such as the userID signifying the creator, the name, a short description, as well as a published field that allows owners to modify the study before requesting experts to participate. The owner table references the study table to allow multiple owners per study. This allows a team of researchers access to the study information and results. The information contained within the remaining tables is useless without the context provided by referencing the study table.

The last of the three core tables is the images table which holds information pertaining to the images. This information includes the image name, corresponding study and the location on the file system of the image. Other tables of note are the savePlace table and the results table. savePlace is a short term data place holder for experts' ranking. This allows individuals the ability to save their progress and resume at a later date. The results table holds the final ranking of each expert for all studies. Both the savePlace table and the results table reference the images table, the study table and the experts table.

\subsection{Site Organization}

Similar to the database, the architecture of the site revolves around three core pages and features: create, rank and results. Other pages are ancillary and supporting of these three features. Figure 3-2 shows the site map.

The site map is organized to show the relationship between pages, as well as the overall flow of the site. A user must register or login before any access to the underlying pages is granted and when complete the user logs out and the session is ended. The map also shows the supporting backend of the site, the database, css, jQuery and two proprietary files in manage.php 
and functions.php. The two proprietary files handle form submissions, Ajax calls and database manipulations. The subsequent sections will discuss each page.

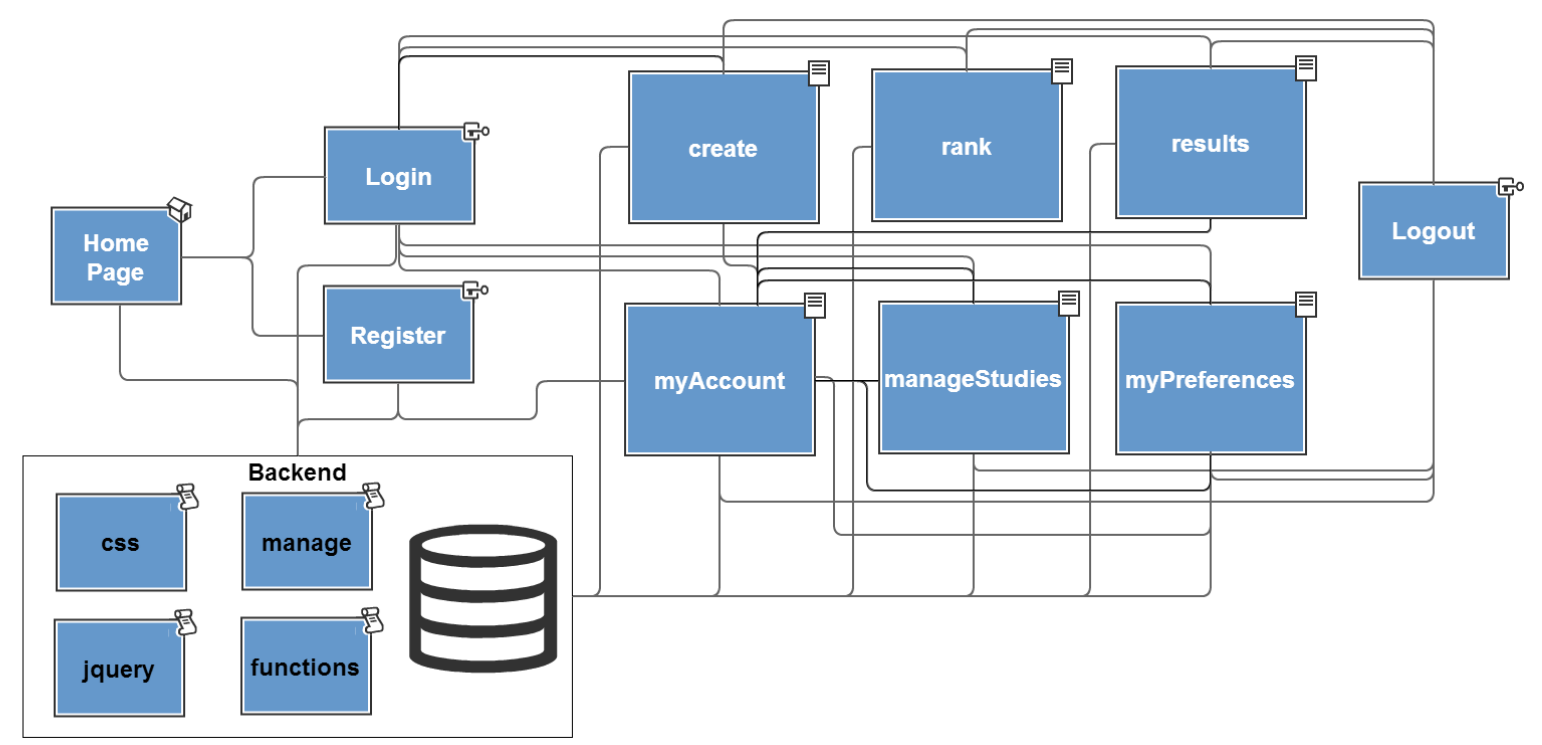

Figure 3-2: Medirank Site Organization

\subsubsection{Create}

Before a study can be ranked by experts or results compiled it must be created; Create.php is the page that handles this function. Implemented on this page are three main features: adding experts to the study, adding properties to images and uploading multiple images.

The first feature of this page is adding experts to the study, which can be done in two ways. The first way is by selecting experts that are registered in the system. To do this the study creator selects the two specialties, a primary and secondary, that best fit the study. If there are users in the system that have selected those specialties as interests, they will appear in a list which the study creator can then select. This is implemented via AJAX and references manage.php. This process is shown in Figure 3-3. 


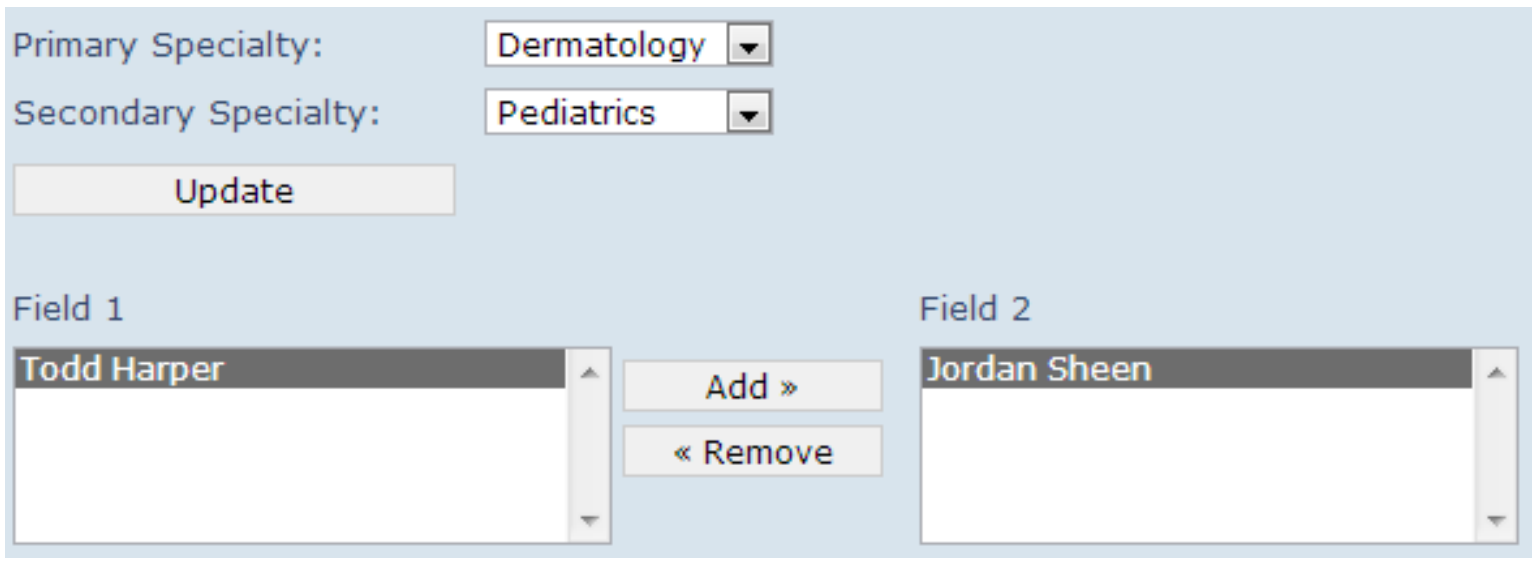

Figure 3-3: Adding Expert in System

The second way that an expert can be added is via a text box as shown in Figure 3-4. To do this the user creating the study must input the first and second name followed by a comma and then the email address. This new expert is then created as a user with a random password which is sent to the expert with instructions to login.

\section{Example: John Smith, jsmith@mail.com}

Todd Harper, tharper@harper.orgl

Figure 3-4: Adding New Expert

The second feature implemented is the ability to upload multiple images. This is done with the assistance of a jQuery plugin named MultiFile.js. The owner can select each image to be uploaded without leaving the page or submitting the form. The name of each image is shown below the upload box. If the study creator wishes to remove an image from the upload pool, that functionality is provided as well via an ' $\mathrm{x}$ ' to the left of the image name. This is shown in Figure 3-5 below. 
Files must be uploaded one at a time. Accepted image types are .jpg and .png Choose File No fil...hosen

004.png

005.png

Figure 3-5: Uploading Images

Once the form is filled out, the study creator selects the Submit button and the form is submitted to manage.php. This page, along with functions.php, creates a database entry for the study with the title, description and study creator as parameters. Next the experts are parsed and added to the database. Finally, the images are checked and uploaded to a directory created for the study. The entire detailed workflow for creating a study is shown in Figure 3-6 below.

It is important to note that at this point the study has not been published. The creator and other study owners still have the ability to edit the details before publishing. This process is discussed in the following section.

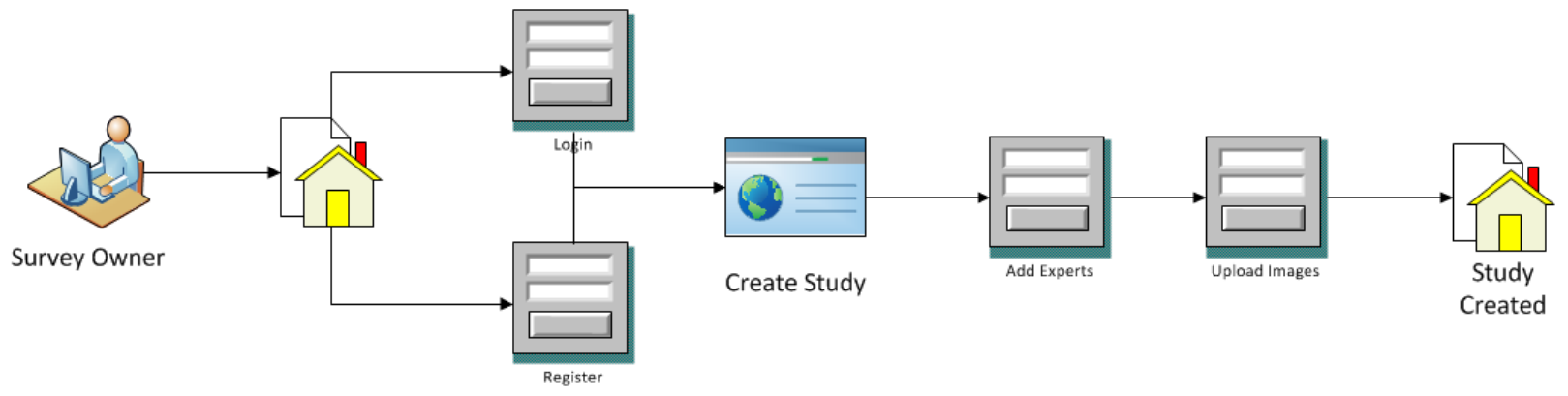

Figure 3-6: Workflow for Creating a Study 


\subsubsection{Manage Studies}

After a study is created the owner can modify it using manageStudies.php. Similar to creating the study, experts can be added, images uploaded and the name and description edited. With the exception of uploading images, these actions can be done at any time. Uploading additional images is prohibited once the study has been published because it would skew the ranking. Publishing the study is done by selecting the 'Publish Study' button which sets a flag in the database that the study is published and then an email is sent to each expert, on behalf of the owner, with a request to rank the images. It is important to note that the experts are not informed who else is participating in the study. When published the page is reloaded and the ability to upload images is dynamically removed. Adding other experts is similar to the process discussed in section 3.4.1.

Setting other users as a study owner is another feature implemented on this page. This feature allows a group of researchers to each have access to the study, monitor the progress and access the final results. Displayed on the page is a list of current study participants as shown in Figure 3-7 below.

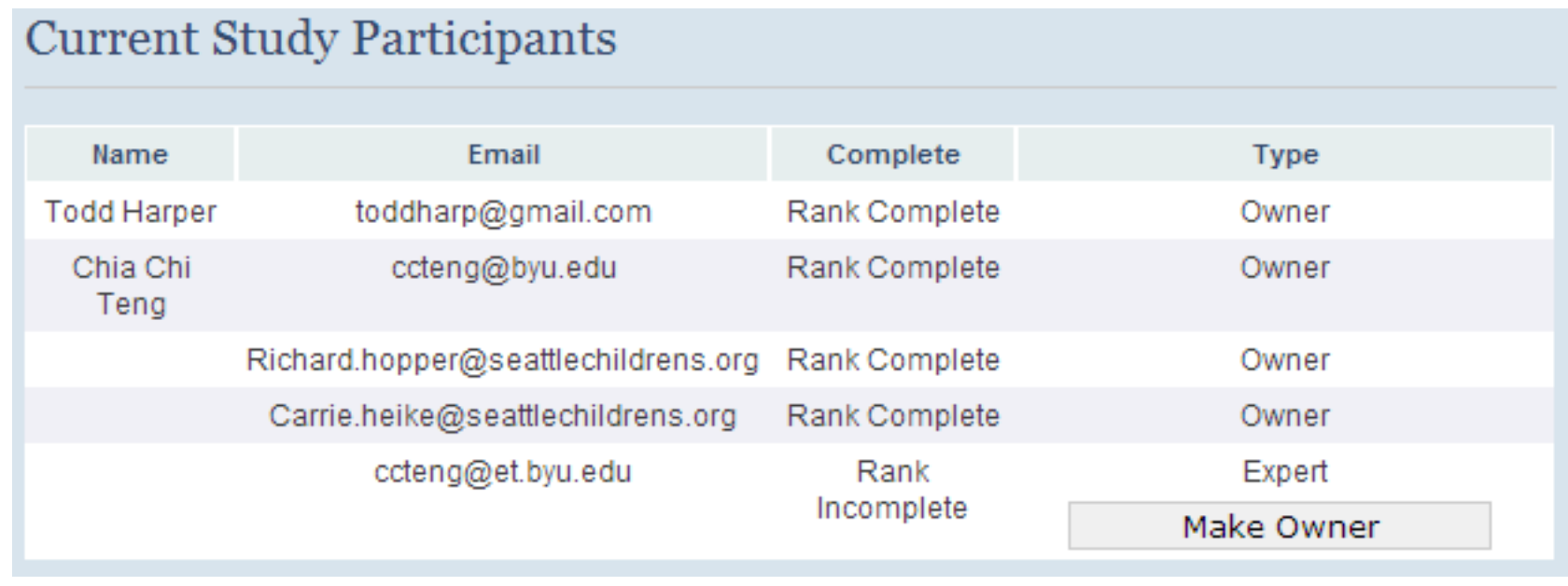

Figure 3-7: Study Participants 
As shown, the table displays each expert's name, email address, progress and type. The progress shows the owner who has completed and has yet to complete the study. The type field shows whether or not the user is an owner or an expert. The system design, as discussed in Section 3.3, allows for a user to be both an expert and an owner on the same study. In Figure 3-7, the final user shown is an expert. Each expert has a button titled 'Make Owner' which allows an owner of the study to set that expert as another owner.

\subsubsection{Rank}

As previously discussed, upon publishing the study the experts are notified via an email with a request to participate in the study. The email consists of the study title, description, a direct link to the study, and for new users a username and temporary password. Once signed in, the expert can begin the process to rank the images using the implemented drag and drop feature, shown in Figure 3-8. At this point in the process, to prevent the participant from being influenced the anonymity of other experts and their ranking is protected.

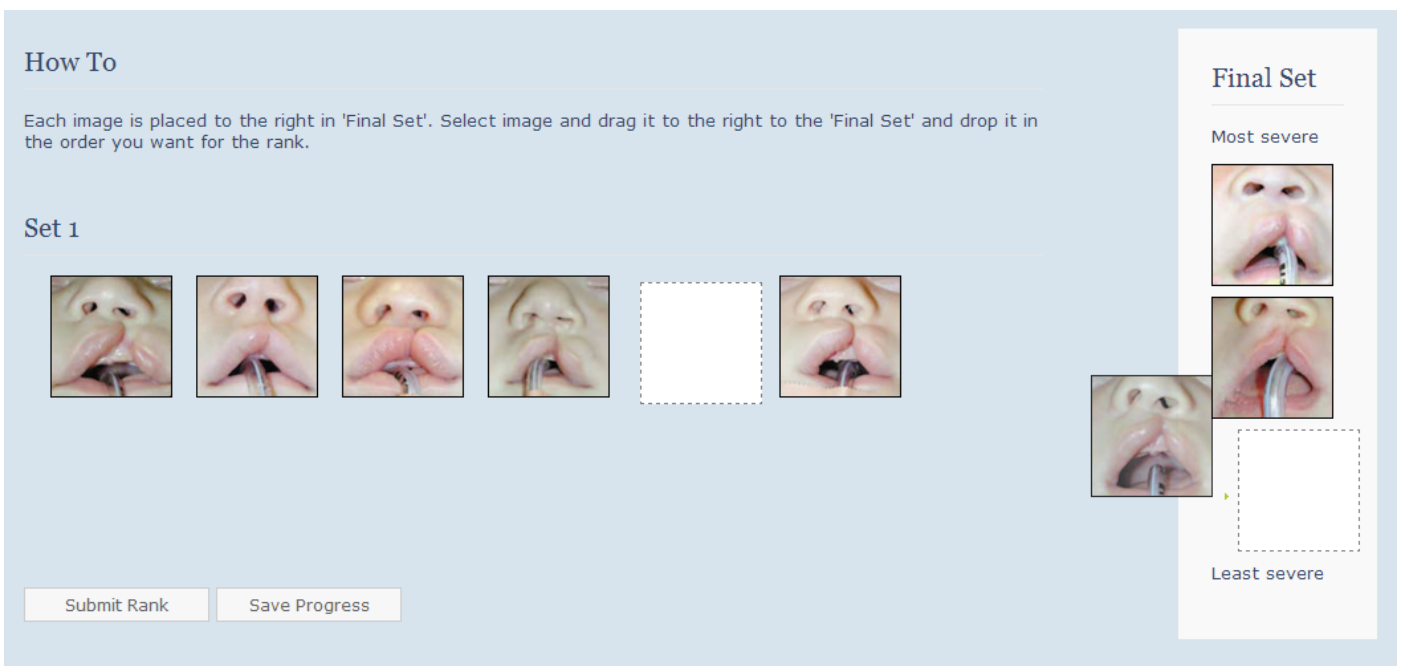

Figure 3-8: Ranking Images 
For statistical purposes, the images are randomly populated into Set 1 in an effort to remove any preconceived rank of the images. The expert selects an image in Set 1 and drags it to the Final Set which is the location of the expert's final ranking. Figure 3-8 shows two squares the size of the image and a dotted border, signifying the possible destinations of the image. The Final Set has a pair of adjectives that serve to help the expert know how to rank. As evident in Figure 3-8 the images are to be ranked from most sever to least severe, with the most severe images at the top column and the least severe at the bottom of the column.

Figure 3-8 also shows another feature of this page, the ability to save your progress. If the expert, for whatever reason, cannot finish the study at that moment they have the option to save their progress. To do so, instead of selecting 'Submit' they select 'Save Progress'. This inserts the current order into the database so that the expert can return and finish the study at another time. However, when the expert completes the study the submit button is pressed and the final ranking is inserted into the database. It is only after submission that the participant is granted limited access to view results.

\subsubsection{Results}

At any point after the study is created, the owners can see the results. The results, compiled in real time when the page is loaded, are based on the overall rank of the images. This is derived by averaging the placement of each image as ranked by the experts. The results page shows the owner several things.

First, the results page shows an ordered list of the images. This is based off the overall ranking of the images and is styled in an image carousel for simple navigation. This allows the researchers to quickly see the order of the images. This carousel is shown in Figure 3-9. 


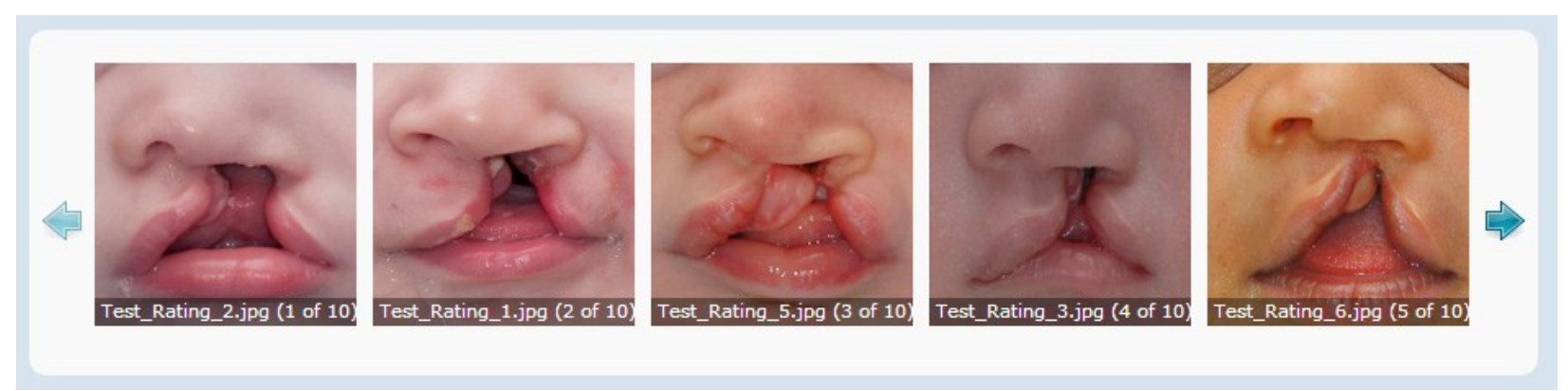

Figure 3-9: Image Carousel

Second, a graph, as shown in Figure 3-10 below, shows the individual rankings by the experts. The $\mathrm{x}$-axis of the graph uses the overall image rank for the ordering. The graph also displays a line representing the correlation of the rankings. Similar graphs for each image property are shown as well. In an effort to preserve anonymity, the participant key as shown in the top right corner of Figure 3-10 is only visible to the study creators.

The third feature shown on the results page is the correlation coefficient for the images. The formula for deriving the correlation is below:

$$
\frac{N \sum X Y-\left(\sum X\right)\left(\sum Y\right)}{\sqrt{\left(\left(N \sum X^{2}\right)-\left(\sum X\right)^{2}\right)\left(\left(N \sum Y^{2}\right)-\left(\sum Y\right)^{2}\right)}}
$$

In this formula, $N$ is the total number of results, meaning the number of images multiplied by the number of experts. $X$ is the rank the individual expert gave while $Y$ is the overall rank of the image. 


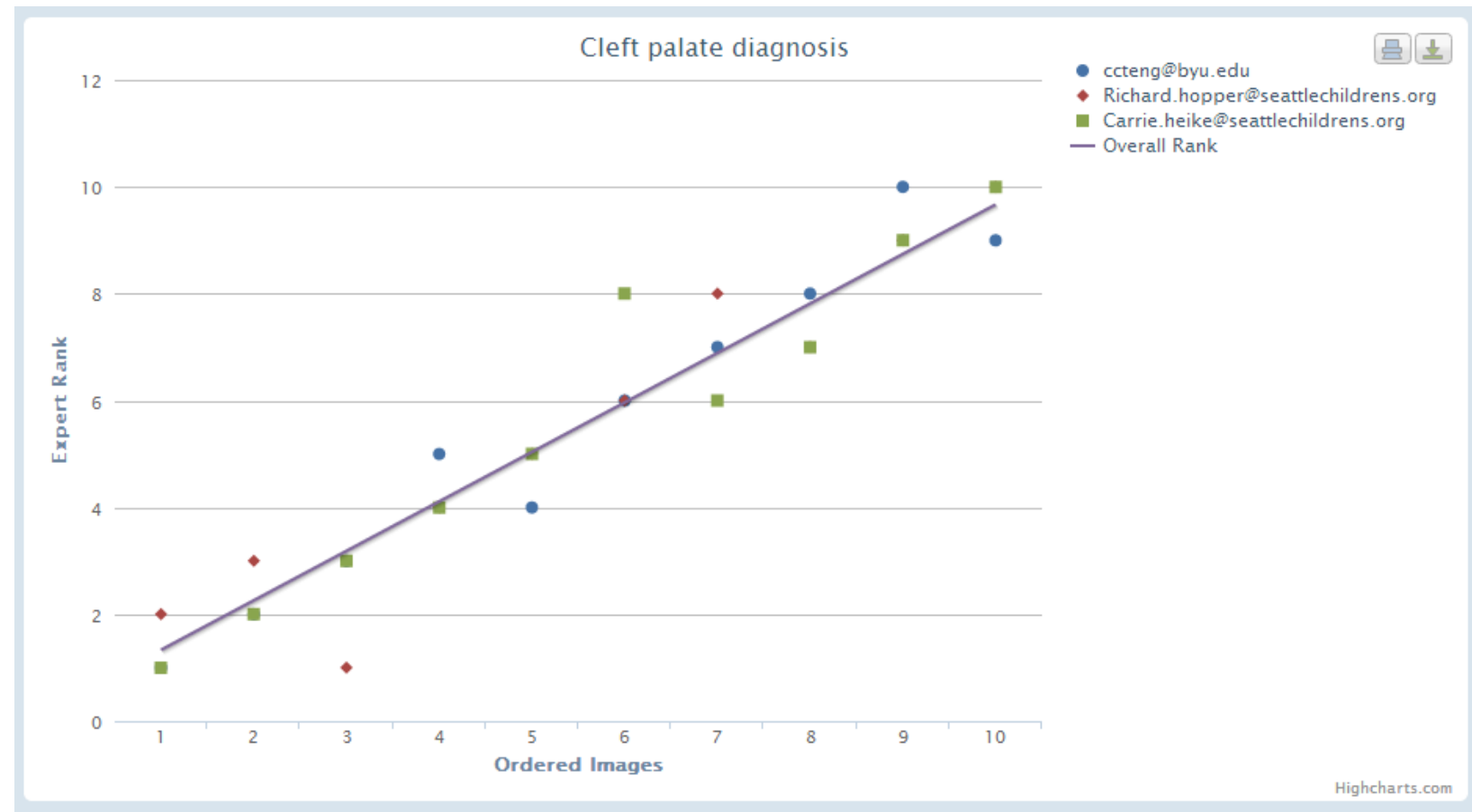

Figure 3-10: Results Graph

The final feature implemented on this page is accessibility to the final rankings. Only the researchers are able to download a CSV of the results to do their own statistical analysis. The results are also shown in a table format on the page. The table columns are able to be sorted so that researchers may view the specific order of the data. This is shown in Figure 3-11.

\subsubsection{My Home}

As each user logs into the site the default landing page is myhome.php. This page has access to each of the other pages and shows the user two tables of information. The first table contains information about the studies that the user owns and contains a link to manage the study and a link to see the results of the study. The second table is similar except it details the studies that the user has participated in as an expert. Listed is a link to complete the study and a link to see a limited view of the results. These two tables are illustrated in Figure 3-12. 


\begin{tabular}{|c|c|c|c|c|c|c|}
\hline Overall Rank & 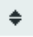 & Image ID $\triangleq$ & Expert Rank & Image Name & Expert & 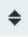 \\
\hline 1 & & 57 & 1 & Test_Rating_2.jpg & ccteng@byu.edu & \\
\hline 2 & & 56 & 2 & Test_Rating_1.jpg & ccteng@byu.edu & \\
\hline 3 & & 60 & 3 & Test_Rating_5.jpg & ccteng@byu.edu & \\
\hline 4 & & 58 & 5 & Test_Rating_3.jpg & ccteng@byu.edu & \\
\hline 5 & & 61 & 4 & Test_Rating_6.jpg & ccteng@byu.edu & \\
\hline 6 & & 63 & 6 & Test_Rating_8.jpg & ccteng@byu.edu & \\
\hline 7 & & 65 & 7 & Test_Rating_10.jpg & ccteng@byu.edu & \\
\hline 8 & & 62 & 8 & Test_Rating_7.jpg & ccteng@byu.edu & \\
\hline 9 & & 59 & 10 & Test_Rating_4.jpg & ccteng@byu.edu & \\
\hline 10 & & 64 & 9 & Test_Rating_9.jpg & ccteng@byu.edu & \\
\hline 1 & & 57 & 2 & Test_Rating_2.jpg & Richard.hopper@seattlechildrens.org & \\
\hline 2 & & 56 & 3 & Test_Rating_1.jpg & Richard.hopper@seattlechildrens.org & \\
\hline 3 & & 60 & 1 & Test_Rating_5.jpg & Richard.hopper@seattlechildrens.org & \\
\hline 4 & & 58 & 4 & Test_Rating_3.jpg & Richard.hopper@seattlechildrens.org & \\
\hline 5 & & 61 & 5 & Test_Rating_6.jpg & Richard.hopper@seattlechildrens.org & \\
\hline 6 & & 63 & 6 & Test_Rating_8.jpg & Richard.hopper@seattlechildrens.org & \\
\hline 7 & & 65 & 8 & Test_Rating_10.jpg & Richard.hopper@seattlechildrens.org & \\
\hline 8 & & 62 & 7 & Test_Rating_7.jpg & Richard.hopper@seattlechildrens.org & \\
\hline 9 & & 59 & 9 & Test_Rating_4.jpg & Richard.hopper@seattlechildrens.org & \\
\hline 10 & & 64 & 10 & Test_Rating_9.jpg & Richard.hopper@seattlechildrens.org & \\
\hline 1 & & 57 & 1 & Test_Rating_2.jpg & Carrie.heike@seattlechildrens.org & \\
\hline 2 & & 56 & 2 & Test_Rating_1.jpg & Carrie.heike@seattlechildrens.org & \\
\hline 3 & & 60 & 3 & Test_Rating_5.jpg & Carrie.heike@seattlechildrens.org & \\
\hline 4 & & 58 & 4 & Test_Rating_3.jpg & Carrie.heike@seattlechildrens.org & \\
\hline 5 & & 61 & 5 & Test_Rating_6.jpg & Carrie.heike@seattlechildrens.org & \\
\hline 6 & & 63 & 8 & Test_Rating_8.jpg & Carrie.heike@seattlechildrens.org & \\
\hline 7 & & 65 & 6 & Test_Rating_10.jpg & Carrie.heike@seattlechildrens.org & \\
\hline 8 & & 62 & 7 & Test_Rating_7.jpg & Carrie.heike@seattlechildrens.org & \\
\hline 9 & & 59 & 9 & Test_Rating_4.jpg & Carrie.heike@seattlechildrens.org & \\
\hline 10 & & 64 & 10 & Test_Rating_9.jpg & Carrie.heike@seattlechildrens.org & \\
\hline
\end{tabular}

Figure 3-11: Table View of Full Data

\subsubsection{My Account}

This final page allows a user to change their account information entered when registering. Users can edit their password, their name and email and also update their interests or expertise. 


\begin{tabular}{|c|c|c|c|c|}
\hline \multicolumn{5}{|c|}{ Studies I Own } \\
\hline \multicolumn{5}{|c|}{ Note: To change study settings select manage. } \\
\hline Name & \multicolumn{2}{|c|}{ Description } & Results & Manage \\
\hline Full Study & \multicolumn{2}{|c|}{ This is a full study. } & $\begin{array}{c}\text { See } \\
\text { Results }\end{array}$ & $\begin{array}{l}\text { Manage } \\
\text { Study }\end{array}$ \\
\hline Partial Study & \multicolumn{2}{|c|}{ This is just a test study for the basis of a test. } & $\begin{array}{c}\text { See } \\
\text { Results }\end{array}$ & $\begin{array}{l}\text { Manage } \\
\text { Study }\end{array}$ \\
\hline $\begin{array}{l}\text { Cleft palate } \\
\text { diagnosis }\end{array}$ & \multicolumn{2}{|c|}{$\begin{array}{l}\text { This study is using images from Dr. Hopper to test the Medirank system } \\
\text { from inside the hospital. }\end{array}$} & $\begin{array}{c}\text { See } \\
\text { Results }\end{array}$ & $\begin{array}{l}\text { Manage } \\
\text { Study }\end{array}$ \\
\hline \multicolumn{5}{|c|}{ Studies I Participated In } \\
\hline Name & Description & Results & \multicolumn{2}{|c|}{ My Ranking } \\
\hline Full Study & This is a full study. & See Results & \multicolumn{2}{|c|}{ Take Survey } \\
\hline
\end{tabular}

Figure 3-12: Landing Page Tables Showing Associated Studies

\subsection{Security}

Because the primary purpose for this survey system is medical research, precautions have been taken to protect the sensitive nature of the data. It is not enough to assume researchers will clear their data of sensitive information before uploading it to the system. The following safeguards have been implemented.

\subsubsection{Images}

Images make or break the site. Prospective users must be able to upload images in order to carry out studies. However, images present a large risk for the site as well as the patients depicted in the images.

The first protective measure implemented is limiting the type of file that can be uploaded. This is done as the file is in the process of being uploaded to the server. First the file extension is checked to ensure it is an accepted extension. Since the extension is purely controlled by the 
client, this cannot be trusted. Checking the extension is an initial weeding out of presented files. Second the mime type of the file is checked by using two functions that read the exif data of the image. The first is by using the PHP function getimagesize which returns several bits of data, one being the mime type of the file. The mime type is then checked against a white list of approved types. The second method is using the PHP function exifimagetype to return the mime type of the image. This is also checked against a white list. Finally the mime types returned by both functions are compared against each other and if the same, are then moved to the image folder.

The second protective measure for images utilizes htaccess files, a feature provided by the Apache webserver. Htaccess files provide a way to make configuration changes on a specified directory instead of system wide. The first implemented change disables PHP parsing and execution in the upload directory and subdirectories. In the event that a malicious PHP disguised as an image is uploaded, the file will not be parsed as PHP and will not execute. The second configuration change made in the htaccess file uses the ForceType directive which forces all files matching a particular pattern to be served with the specified mime content-type. This forces all files that end with a .jpg extension to be served with that mime type, effectively ensuring that no malicious scripts are run on the server.

The third protective measure for images removes the exif data of the image. As shown in Chapter 2, images contain more data than the image itself. This exif data contains the date and time the image was taken, the type of camera and lens used, as well as a geolocation where the image was taken. In order to preserve the privacy of the patients in the images, all exif data is removed. This is achieved by using the open source exiftool. This program, written in Perl, is executed on each image after upload. The clean images are then presented to the users for ranking and results. 


\subsubsection{User Input}

In addition to images, users are able to input some text, namely the study name and the study description. Accepting user text input exposes the possibility of cross site scripting and SQL injection. Mitigation of these threats is done two ways. First the data is sanitized using an open source package "htmlpurifier" which works before the data is inserted into the database and after the data comes out of the database to remove any malicious data that is used for cross site scripting. Second, to prevent SQL injection, all database queries use PHP Data Objects (PDO) which provides an object layer between PHP and the database. Prepared statements prevent secondary queries from being run so that attempts to inject SQL into user input would not succeed.

\subsubsection{Other}

Other, less user-impacting security measures have been implemented. The first is permissions on the server. Out of necessity, the files that are executed for the survey system have access to stored data; however, it is important that only certain files retain access. Scripts should not be executed as a root user. Each file within the web directory is owned by the web server user and no other user, besides root, can execute the files. The files also cannot be executed using root level permissions.

A second implementation is the use of HTTPS throughout the site. HTTPS encrypts the data between the client and the server. This is especially important for password reset, user login and user registration. The encrypted nature of the data prevents a third party from knowing what is transferred. 


\section{RESULTS AND ANALYSIS}

This chapter details the various tests and studies which were conducted on the created site; usability studies, to test the actual site, and comparison studies, to test the hypothesis. The two usability studies provided valuable feedback for the improvement of the ranking process and page layout. The comparison studies suggest that the web-based method is an improvement over the paper-based method in four of the five tested areas.

\subsection{Usability Study 1}

The first case study was conducted with two lead physicians at Seattle Children's Hospital. This study is intended to show the functionality of the site as well as gain feedback from the intended user base.

While the system worked, there were some unforeseen errors that caused confusion. The users in this case study felt that ranking from a grid system to a single column was difficult and confusing. This was caused by too much scrolling in the process of ranking. Another problem with the vertical ranking was that there were no indicators on how to rank the images. One user ranked the images from least to most severe while the other ranked from most to least severe. Obviously, this skewed the results. The instructions also were unclear about what the user needed to accomplish. Finally, the users requested the ability to add properties to the images. 


\subsection{Usability Study 2}

In preparation for the second case study, feedback from the first case study resulted in the addition of adjectives indicating how the images were to be ranked. The second case study was conducted to show the functionality of the site. A study was created with 40 images of infants with cleft palates. Twenty individuals were added to the study and requested to participate. It is important to note that for this study none of those petitioned to participate in the study are experts in the medical field. There is a variance of education, profession, gender and age. After the participants ranked the images, they were directed to an eight question survey about their experience. They were asked to answer the questions using a five point Likert scale, with 1 being Strongly disagree and 5 being Strongly agree. 12 of the 20 people participated.

The first question in the experience survey was about the instructions given on the ranking page. $75 \%$ of the participants agreed that the instructions were adequate, $16 \%$ disagreed and $8 \%$ had no opinion.

Question two concerns the process of dragging and dropping the images. Of the participants, $64 \%$ agreed that dragging and dropping was intuitive and easy to understand. A full $25 \%$ of participants disagreed with that statement, with the final $8 \%$ responded with no opinion.

Question three of the survey discusses the size of the images presented to be ranked and the impact that size has on proper classification. Of the group, $64 \%$ felt that the image size was not a limiting factor in ranking. Of the remaining participants, $25 \%$ had no opinion on the image sizes with $8 \%$ agreed that the image size made it difficult to rank.

Question four asks the participants whether or not the adjectives used in indicating how to rank the images was helpful. Every participant either agreed or strongly agreed that these adjectives helped them understand how to order the images. 
Question five and question six are linked. Question five asks if the participant attempted to save their progress, of which $42 \%$ responded yes. Question six then asks those responding yes if the functionality worked as they thought it should. Of those that responded yes to question 5, $80 \%$ felt the functionality worked as they expected with $20 \%$ disagreeing with that statement.

Question seven asks the participant if they were satisfied with their overall experience. Half of the responders felt they had a satisfactory experience, $33 \%$ felt their experience was unsatisfactory and $17 \%$ had no opinion.

The final question of the survey asked the participants to share any other details about their experience. Though the comments varied, the majority were concerning the placement of the image sets. As in the first study, most respondents felt that ranking the images in a vertical format was difficult and confusing. Suggestions for remedying that aspect of ranking ranged from being able to sort in place within the first grid, to having the grid scroll with the user as they scroll down the page.

\subsection{Analysis of Usability Studies}

Both case studies showed the functionality of the site. Studies were created, images uploaded, experts added and ranking completed. This proof of functionality does not come without problems. While both case studies showed that ranking can and does take place, the users found it difficult to perform the task.

The first issue with ranking concerned the instructions given on the page. In the two case studies, the instructions read: "Each image is placed to the right in 'Final Set'. Select image and drag it to the right to the 'Final Set' and drop it in the order you want for the rank." The survey conducted in the second case study suggests that $16 \%$ of users felt the instructions were unclear. 
Because of this confusion, the instructions were clarified. Instead of a long sentence, the instructions are written as a list of tasks. The instructions now read as follows:

1. Review each image.

2. Select an image and drag below to the 'Ordered Set'

3. Re-order the images as needed within the final ordered set.

4. Save progress if desired. Submit when complete.

The vertical ranking paradigm caused further confusion. Both studies showed multiple problems with that method of ranking. The first issue was vertical scrolling. Across the board in the second case study the users stated that with such a large number of images, accurate ranking was difficult. One user stated, "The dragging and dropping of the images was clunky when the list started getting longer and you had to put images at the bottom." Another said, "It was really difficult to rank so many images in a vertical layout. It was fine to begin with, but once you have to scroll it was a pain. If there were less images or another layout it would have made it a lot better." This fits with the request of the doctors used in the first case study. They requested that the layout be changed from a vertical system to a horizontal system. The horizontal layout expands and contracts depending on the number of images placed in the set. The CSS overflow property is set to hidden which causes a scroll bar to appear. This allows the user to scroll horizontally without affecting the other elements on the page. The newly implemented method of ranking horizontally is shown below in Figure 4-1.

Another change shown in Figure 4-1 is the placement of the adjectives which are designed to assist the expert in the way to rank the images. Figure 4-1 shows the ranking from most severe on the left to least severe on the right, instead of most severe on the top to least severe on the bottom as shown in Figure 3-8. The previous design caused confusion for some 
users because they attempted to submit the most severe and least severe images in pairs. The new design indicates that there is a flow to how all the images should be placed before submission.

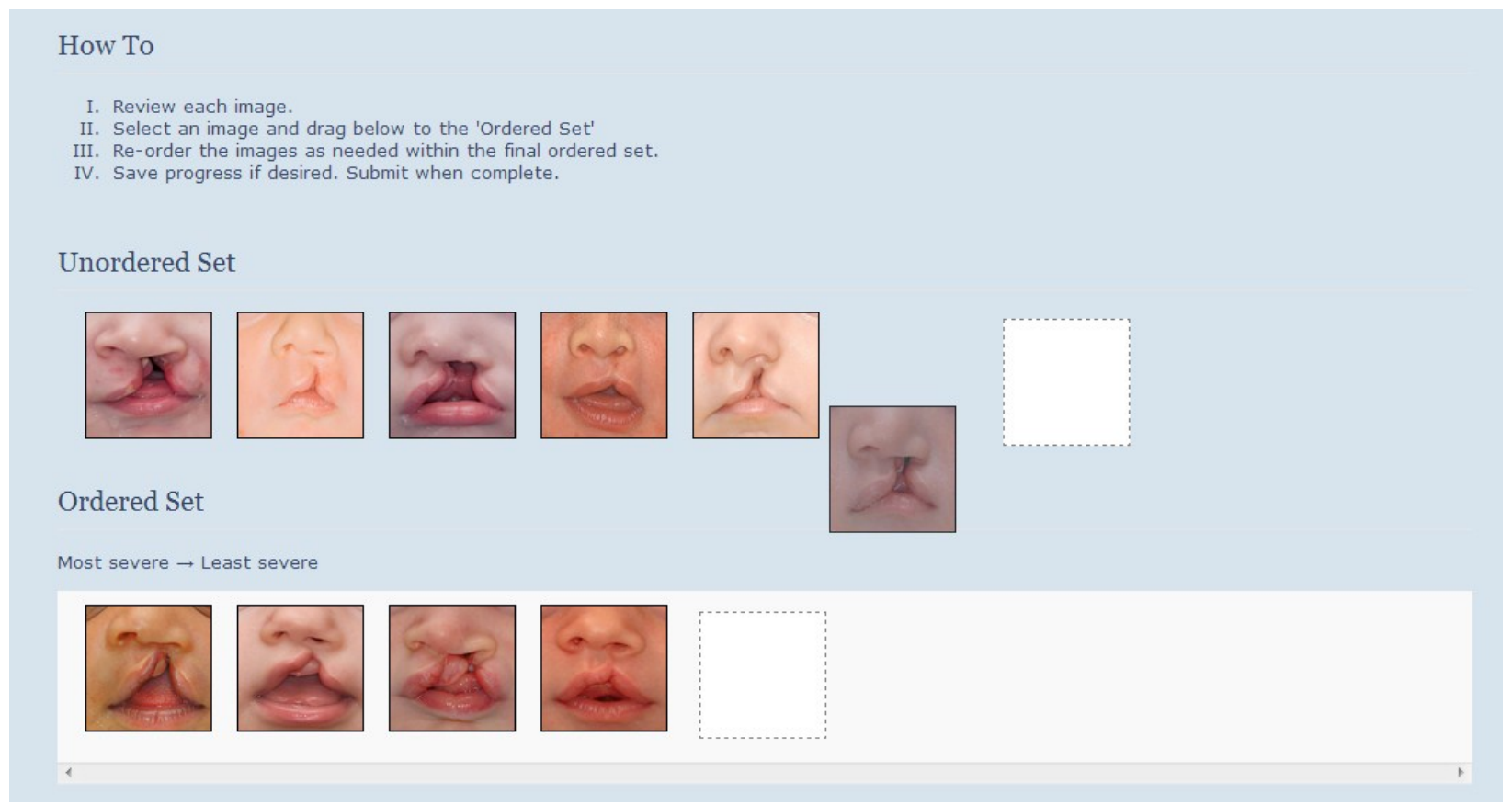

Figure 4-1: Redesigned Rank Layout

The final addition to the site is the ability to add properties to the images. This feature was specifically requested by the users in the first case study. It allows the study creator to add more detailed properties to the images and to collect statistics of the images based on those properties. For example, some images in a set might have a particular genetic disorder. The owner could then determine if the genetic disorder has any bearing on the final ranking of the images. The process of adding properties is similar to adding an expert as discussed in Section 3.4.1. The properties are presented as a list from which the study creator can select the desired properties and then add them to the study. This is shown in Figure 4-2 below. 


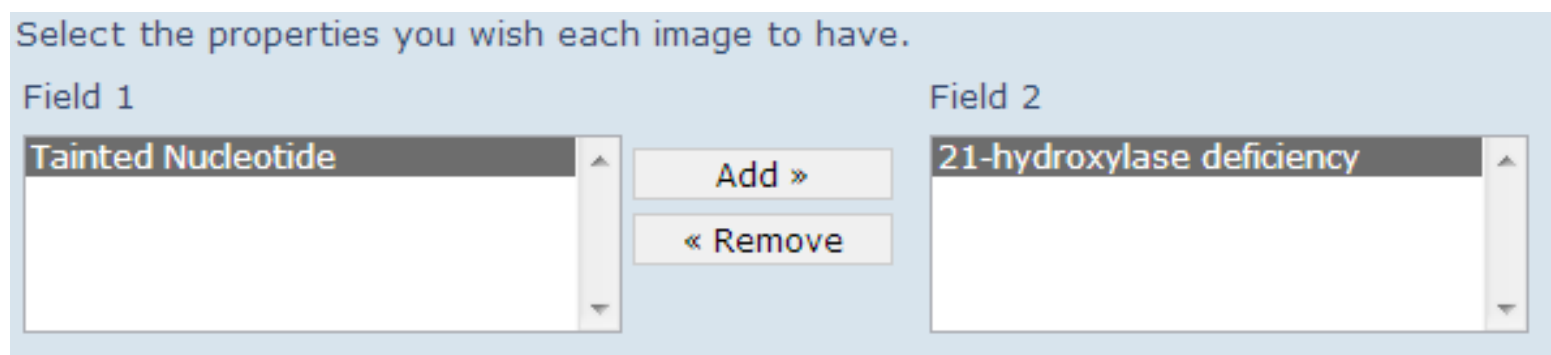

Figure 4-2: Adding an Image Property

Once added, the image properties must be edited. Each image and associated properties appear in a table. Here the study owner can edit each property, adding or removing details as desired. When finished the entire table is submitted and saved. Editing an image property is shown in Figure 4-3 below.

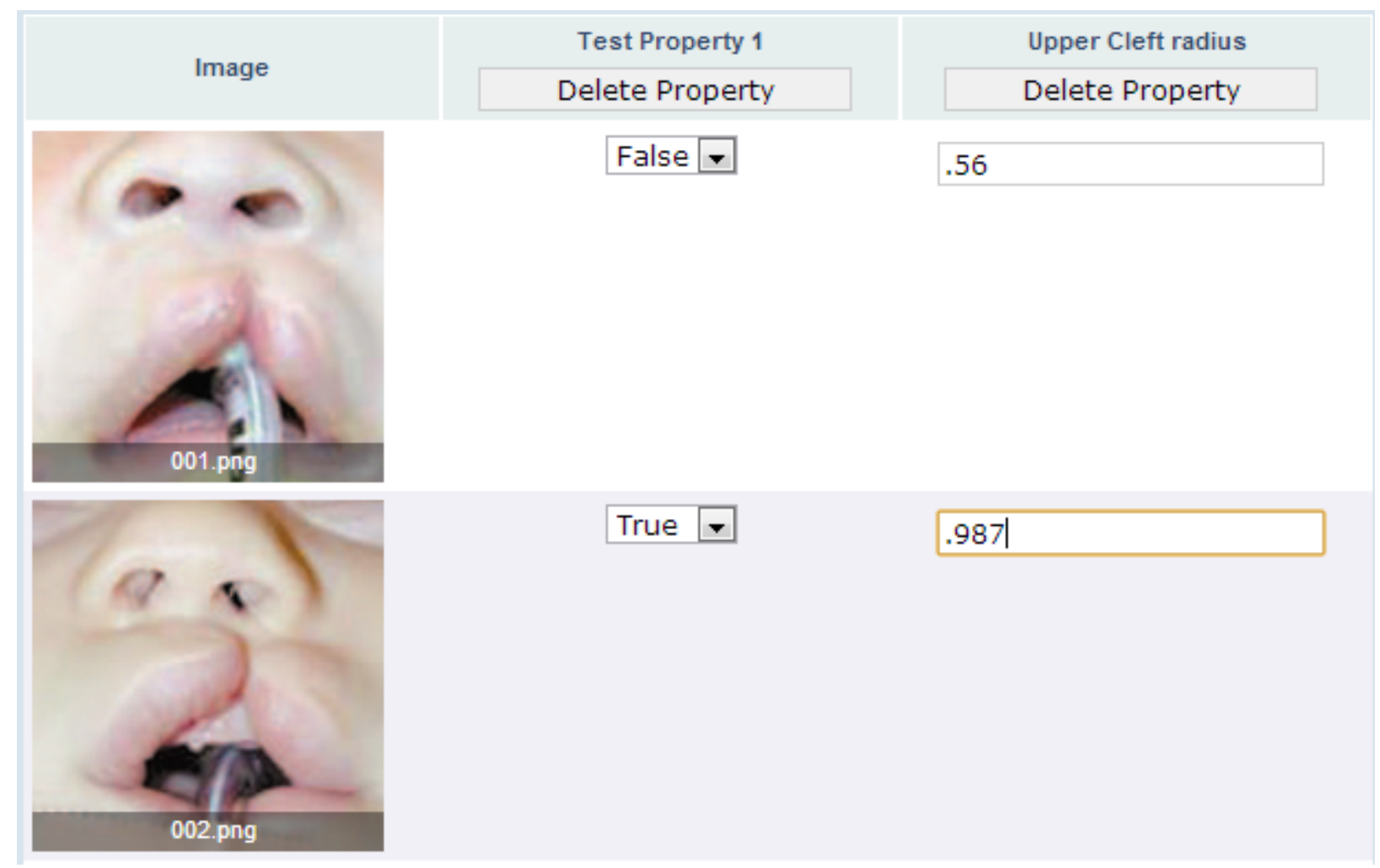

Figure 4-3: Editing an Image Property 


\subsection{Comparison Study 1}

The first comparison study focuses on $\mathrm{H} 2$, cost to the researcher, and $\mathrm{H} 3$, response rate, from the hypothesis. Cost to the researcher is measured in dollars spent administering the study and response rate is measured by how many participants complete the image ranking. To judge these two points, 20 total participants were randomly divided into two groups to rank 20 images. Group 1 ranked the images using the online method while Group 2 ranked the images using the paper method administered by mail. 18 of the participants were located throughout the United States while 2 participants were located internationally. Each group held one international participant. Both groups were given the same images to rank as well as the same short questionnaire regarding their experience.

As stated, cost to the researcher is measured in the dollars spent in administering the study. The study includes postage, both to and from the recipients in group 2 , and ink for the high quality color images. The United States Postal Service was used to send the packages to each recipient both domestically and internationally. The domestic total for postage was $\$ 55.47$ and international postage to Santiago Chile was $\$ 8.01$. The printer used in printing the images was a Brother LC61 series. On Amazon.com the official Brother ink for this model is listed at \$41.01 (Brother LC-61 4-Color Ink Cartridges n.d.). Since each full image set used only two sheets of paper for a total of 20 sheets, the cost of the paper is not used in this calculation. The total cost for the paper-based study is $\$ 104.90$. In contrast, the web-based study incurs zero cost to the researcher since the web application is free to use.

Response rate is measured by how many participants in each study complete the task of ranking the images within the specified time frame of three weeks. Each group consisted of ten participants with one participant in each being international. Group 1 had a response rate of 7 out 
of 10 . Group 2 had a response rate of 4 out of 10 . Of the respondents in group 1, the international participant completed the study, while the international participant in group 2 did not respond.

Interestingly, in the questionnaire $100 \%$ of the respondents replied that in similar studies in the future they would prefer an online method of ranking images over the paper-based.

\subsection{Comparison Study 2}

This second comparison study is designed to test three elements of the hypothesis, namely: $\mathrm{H} 1$, time to task completion; $\mathrm{H} 4$, method preference; and $\mathrm{H} 5$, user satisfaction. This study used participants from a class in the Brigham Young University Information Technology department. The students were placed into two groups to rank a set of 20 images using both the web-based and paper-based methods. Group 1, consisting of 10 participants, ranked the images online first then offline while group 2, with 11 participants, ranked the images offline then online. When finished ranking the images, the students were given a short survey about their experience and preferences.

H1 states that the task of ranking the images would be faster online than offline. The students were instructed to record their start and stop times for each task. The times were then recorded and averaged for each method. In both groups the offline paper-based method resulted in a faster time to completion.

H4 states that the method of preference will be the online method and not the offline method. It should be noted that the participants are preparing to enter into the information technology field and that their chosen profession could impact their views. Overall the majority of participants preferred the online method over the offline. 
H5 surmises that overall, users will be more satisfied with the online experience than the offline. This was measured through the questionnaire by asking all participants to rate their satisfaction levels of each method. Overall a greater majority of people were satisfied with the web-based method than those who were satisfied with the paper-based. For full details see Table 6 in section 4.7.The participants described the paper-based method as "straight forward", "the order was hard to define", "more tangible", "quick but hard to get an overall view", "fairly easy to sort", and that it "needed space". The participants described the web-based method as "more convenient", "less messy", "easy to use", "easier and faster", "UI is simple", "UI is buggy”.

When asked which method they would be most likely to respond to in the future, $90 \%$ indicated online and 10\% indicated offline. The reasons for online include "easier to do it on a computer", "more convenient and less messy", "faster", "easier to access from anywhere". The reasons given for preferring the offline method are "I liked it better to hold them" and "offline is faster".

\subsection{Analysis of Comparison Studies}

Both comparison studies suggested that the online method of ranking images is an improvement over the offline method in four of the five areas listed in the hypothesis. Comparison study 1 tested $\mathrm{H} 2$ and $\mathrm{H} 3$ and comparison study 2 tested H1, H4 and H5.

H1. Time to task completion: Comparison study 2 shows that the offline method of ranking images is faster than the online method. Table 1 shows the recorded times. 
Table 1: Response Rate

\begin{tabular}{c|cc}
\hline & Online & Offline \\
\hline Overall & $3.32 \mathrm{~min}$ & $2.83 \mathrm{~min}$ \\
Group 1 & $3.6 \mathrm{~min}$ & $2.75 \mathrm{~min}$ \\
Group 2 & $3.06 \mathrm{~min}$ & $2.91 \mathrm{~min}$ \\
\hline
\end{tabular}

The disparity in times for group 1 could be explained that the participants had already seen and ranked the images once, and therefore were able to rank the offline images faster. However, group 2 ranked the images offline and then online, and offline still out performed online. This would suggest that regardless of the online/offline testing order, the offline method is faster to complete the ranking; however, to accurately judge which method is consistently faster, another study should be conducted where the groups rank a different image set per method.

H2. Cost: Cost to the researcher was measured in comparison study 1, where the monetary costs to produce and distribute the study were recorded.

Table 2: Cost

\begin{tabular}{c|cc}
\hline & Online & Offline \\
\hline Cost & $\$ 0$ & $\$ 104.90$ \\
\hline
\end{tabular}

As the number of images and the number of participants increase, the offline cost will also increase. Geographic location of the participants is another factor that can change the cost of a study. Comparison study 1 demonstrates how the inclusion of an international participant can greatly affect the cost. In some cases, such as limited funding, research efforts could be stunted or compromised in their ability to conduct a full study. Because the online method is presented to 
the researcher as a free service, the cost remains the same regardless of the number of images, participants, and their geographic location. In the case of cost, online is clearly more advantageous than offline.

H3. Response rate: Response rate, as measured in comparison study 1, was defined by how many participants in the study actually returned a completed study within the specified time frame. The response rate is shown in Table 3 below.

Table 3: Response Rate

\begin{tabular}{l|cc}
\hline & Group 1 & Group 2 \\
\hline Response Rate & $70 \%$ & $40 \%$ \\
\hline
\end{tabular}

The difference in response rate was influenced by the following factors.

1. The online method has the advantage of sending reminder emails to participants, which resulted in more responses.

2. The offline method had two surveys undelivered because the address labels were missing. Though the cause is undetermined, this was either an error by those conducting the study or an error on the part of the postal service. Despite where the fault lies, this emphasized the difficulties that could arise from simple human error.

3. The international participant in group 2 did not even receive the packet in time to complete and return the study in the specified time frame. The remaining participants, regardless of their group, simply did not respond. Both methods have short comings; however, comparison study 1 would indicate that the web-based studies result in a higher response rate than their paper-based counterparts and pose less logistical issues. 
H4. Method Preference: Method preference was measured by asking the participants through a questionnaire which method they preferred. In comparison study 2 , the majority of the respondents preferred the online method over the offline method as shown in Table 4 .

Table 4: Study 2 Method Preference

\begin{tabular}{c|cc}
\hline & Online & Offline \\
\hline Overall & $67 \%$ & $33 \%$ \\
Group 1 & $60 \%$ & $40 \%$ \\
Gorup 2 & $73 \%$ & $27 \%$ \\
\hline
\end{tabular}

As previously noted, the participants in this study were students of Information Technology. This could show bias in their preference. To counter this, participants in comparison study 1, who were diverse in age, vocation, and location, were asked a similar question regarding their preference, shown in Table 5.

Table 5: Study 1 Method Preference

\begin{tabular}{c|cc}
\hline & Online & Offline \\
\hline Overall & $100 \%$ & $0 \%$ \\
Group 1 & $100 \%$ & $0 \%$ \\
Group2 & $100 \%$ & $0 \%$ \\
\hline
\end{tabular}

Since those participating took part in either the online or the offline method, not both, the question was "Which method of completing a similar study would you prefer?" The results of the two studies suggest that a majority of users prefer an online method over an offline method. Further research could be done to test preferences over a wider demographic. 
H5. User satisfaction: Satisfaction was measured by asking the participants in comparison study 2 to rate their overall satisfaction for each of the two methods. While users showed satisfaction in both methods the web-based method received a much higher percentage of users in the range of satisfied to very satisfied than the paper-based method. Again, the participants are technology students and bias could be a factor. At the same time, satisfaction with one method does not preclude satisfaction with the other. The collected data suggests that the online method produces a higher satisfaction rating than that of the offline method.

Table 6: User Satisfaction Rating

\begin{tabular}{c|cccccc}
\hline & \multicolumn{2}{|c}{ Overall } & \multicolumn{2}{c}{ Group 1 } & \multicolumn{2}{c}{ Group 2 } \\
\cline { 2 - 7 } & Online & Offline & Online & Offline & Online & Offline \\
Very Dissatisfied & $0 \%$ & $0 \%$ & $0 \%$ & $0 \%$ & $0 \%$ & $0 \%$ \\
Dissatisfied & $10 \%$ & $5 \%$ & $10 \%$ & $0 \%$ & $9 \%$ & $9 \%$ \\
Neutral & $14 \%$ & $62 \%$ & $20 \%$ & $40 \%$ & $9 \%$ & $82 \%$ \\
Satisfied & $62 \%$ & $33 \%$ & $60 \%$ & $60 \%$ & $64 \%$ & $9 \%$ \\
Very Satisfied & $14 \%$ & $0 \%$ & $10 \%$ & $0 \%$ & $18 \%$ & $0 \%$ \\
\hline
\end{tabular}

Additionally, the questionnaire included an open-ended comment section. Overall, their comments echoed their higher satisfaction with the online study. For a more definitive conclusion on satisfaction and the underlying reasons, a wider demographic should be tested.

Other observations: The data retrieved from each group's ranking resulted in a correlation coefficient, shown in Table 7. Across the two groups the online correlations were consistent, while the offline were not. Within group 1 the correlations between the two methods were very different while the correlation across the two methods was consistent for group 2. 
Table 7: Correlations

\begin{tabular}{c|cc}
\hline & Online & Offline \\
\hline Group 1 & 0.868 & 0.586 \\
Group 2 & 0.939 & 0.911 \\
\hline
\end{tabular}

While it is unclear what the exact reason is for the large difference in group 1's correlation, it can be speculated that the large difference in time to complete the task is partly responsible. For this same group, the time difference between the two methods was 51 seconds. In order to show the link between speed and correlation, more tests should be run with multiple sets of pictures to preserve the unfamiliarity with the images.

In conclusion, the two comparison studies show that the offline method is more efficient in time to task completion; however, they show that the online method is superior in cost to the researcher, response rate, method preference, and user satisfaction.

\subsection{Security Review}

The website was tested by the BYU Cyber Security Research Lab Red Team. A virtual machine of the system was given to the team for their test. Information was gathered using port scans, vulnerability scans, and web browsing. With this information, exploits were attempted which include SQL injection, XSS injection, URL manipulation, and malicious file upload. Although the team was unable to exploit the system, they recommended enabling website cookies to be set to HTTP-Only to further mitigate against cross site scripting attacks. This setting has been changed in the php.ini file on the site. The full report is found in Appendix A. 


\section{CONCLUSIONS AND RECOMMENDATIONS}

\subsection{Review}

Previous research shows the advantages of using Delphi studies to cover gaps in knowledge. It also indicates that the majority of such studies are conducted in text-based systems. Image-based surveys have been used to show the reliability and accuracy of expert ranking. This thesis details the creation and implementation of a web application for the use of creating, managing and ranking image-based Delphi studies. Two case studies were completed showing the functionality of the application. The hypothesis, tested by two comparison studies, resulted in the online method being better than the offline method in cost to the researcher, response rate, method preference, and user satisfaction. The offline method bested the online method in time to task completion.

\subsection{Recommendations for Action and Study}

The implemented site is in its infancy. More researchers must use the application for it to remain relevant. Improvements to the site must take place in added functionality as well as design. A full design study will expose the areas that need improvement so that the user experience is enhanced.

The tests of the hypothesis resulted in areas for future study. One area is the connection of correlation and speed to rank the images. Does speed lower the correlation? Another area for 
further research is the satisfaction with the two methods. What causes the satisfaction and how different is the satisfaction among a more general populace? Finally, a larger demographic should be tested to definitively understand the preference between the two methods.

Though not examined in this thesis, the potential exists to integrate text-based Delphi studies into the site. Adding this feature would give researchers all over the world one place to conduct their Delphi studies. Along with integrating text-based studies, the ability to conduct multiple rounded studies is important since true Delphi studies are conducted over multiple rounds. Multiple rounds allow the researcher to use one study rather than creating a new one for each round.

Another recommendation for further work is integrating touch capability to the site. Touch-based interfaces are rapidly growing in popularity and mobile devices are quickly becoming the norm. Adding in functionality for touch devices frees the researcher as well as the experts from sitting at a desk to complete the study. With touch integration the site can truly become cross platform.

A final recommendation is to port the site to a web framework. The current version is built from the ground up using PHP. Web frameworks remove many of the manual processes such as creating and interacting with the database, form handling or session management. Many web frameworks are object-based, which grants a layer of security through data abstraction and sanitation as well as grants easy access to the database without the need for complex queries. 


\section{$5.3 \quad$ Closing}

Health information technology is an area that will continue to expand. Its use will enhance the care given to those who need it. This research shows that web applications, such as this, can be used to further the research in the medical field which leads to better caregiver training and better clinical guidelines. 


\section{REFERENCES}

Beretta, R. "A Critical Review of the Delphi Technique." Nurse Researcher, 1996: 79-89.

Blumenthal, D., and J. Glasser. "Information Technology Comes to Medicine." The New England Journal of Medicine, 2007: 2527-2534.

Brother LC-61 4-Color Ink Cartridges. n.d. http://www.amazon.com/Brother-LC-61-4-ColorInk-Cartridges/dp/B001DRIDDK/ref=sr_1_3?s=electronics\&ie=UTF8\&qid=1361335347 (accessed February 19, 2013).

Crisp, J., D. Pelletier, C. Duffield, A. Adams, and S. Nagy. "The Delphi Method?" Nursing Research, 1997: 116-118.

Delbecq, A., A. Van de Ven, and D. Gustafson. Group Techniques for Program Planning: A Guide to Nominal Group and Delphi Processes. Glenview: Scott, Foresman and Co., 1975.

Field, M., and K. Lohr. Guidelines for Clinical Practice. Washington, DC: National Academy Press, 1992.

Fisher, D., R. Tse, and J. Marcus. "Objective Measurements for Grading the Primary Unilateral Cleft Lip Nasal Deformity." Journal of Plastic and Reconstructive Surgery, 2008: 874880 .

Goodman, C. "The Delphi Technique: A Critique." Journal of Advanced Nursing, 1987: 729734.

Gralnek, I., R. Defranchis, E. Seidman, J. Leighton, P. Legnani, and B. Lewis. "Development of a Capsule Endoscopy Scoring Index for Small Bowel Mucosal Inflammatory Change." Alimentary Pharacology and Therepeutics, 2008: 146-154.

Hasson, F., S. Keeney, and H. McKenna. "Research Guidelines for the Delphi Survey Technique." Journal of Advanced Nursing, 2000: 1008-1015.

International Standards Organization. "Ergonomic Requirements for Office Work with Visual Display Terminals (VTDs); Part 11 - Guidance on Usability." ISO 9241-11. 1998. 
Lindeman, C. "Delphi Survey of Priorities in Clinical Nursin Research." Nursing Research, 1975: 434-441.

Linestone, H., and M. Turoff. The Delphi Method: Techniques and Applications. Reading: Addison-Wesley, 1975.

McKenna, H. "The Delphi Technique: A Worthwhile Approach for Nursing?" Journal of Advanced Nursing, 1994: 1221-1225.

Mead, D., and L. Moseley. "The Use of Delphi as a Research Approach." Nurse Researcher, 2001: 4-37.

Murphy, M., et al. "Consensus Development Methods, and Their Use in Clinical Guideline Development." Health Technology Assessment, 1998: 1-88.

National Health and Medical Research Council. A Guide to the Development, Implementation and Evaluation of Clinical Practice Guidelines. Canbera: National Health and medical Research Council of Australia, 1998.

Powell, C. "The Delphi Technique: Myths and Realities." Journal of Advanced Nursing, 2003: 376-382.

Qing, L., J. Haiteng, L. Haiyan, L. Gang, T. Gaojun, and Y. Zhijian. "Depression Severity Evaluation for Female Patients Based on a Functional MRI Model." Journal of Magnetic Resonance Imaging, 2010: 1067-1074.

Rennie, D. "Consensus Statements." New England Journal of Medicine, 1981: 665-666.

Rowe, G., G. Wright, and F. Bolger. "Delphi: A Reevaluation of Research and Theory." Technological Forecasting and Social Change, 1991: 235-251.

Sackman, H. "The Problems of Rigor in Qualitative Research." Advances in Nursing Sciences, 1975: 27-37.

Schulman, S., A. Eelde, M. Holmstrom, G. Stahlberg, J. Odeberg, and M. Blomback. "Validation of a Composite Score for Clinical Severity of Hamophilia." Journal of Thrombosis and Haemostasis, 2008: 1113-1121.

Thomas, T., D. Anderson, J. Marsh, and T. Brown. "A Method for the Estimation of Normative Bone Surface Area to Aid in Objective CT-based Fracture Severity Assesment." The Iowa Orthopaedic Journal, 2008: 9-13.

Tullis, T., and B. Albert. Measuring the User Experience: Collecting, Analyzing and Presenting Usability Metrics. New York: Morgan Kaufman, 2008. 
Usability Professionals' Association. What is Usability - UPA Resources. n.d. http://www.upassoc.org/usability_resources/about_usability/definitions_of_usability.html (accessed April 21, 2001).

Van der Linde, H., C. Hofstad, J. Van Limbeek, K. Postema, and J. Geertzen. "Use of the Delphi Technique for Developing National Clinical Guidelines for Prescription of Lower-limb Prostheses." Journal of Rehabilitation Research and Development, 2005: 693-704.

Vogt, P., F. Nentwich, N. Jovanovic, E. Kirda, C. Kruegel, and G. Vigna. "Cross Site Scripting Prevention with Dynamic Data Tainting and Static Analysis." Network and Distributed Ssytem Security Symposium. Sand Diego: NDSS, 2007.

Woolf, S., R. Grol, A. Hutchinson, M. Eccles, and J. Grimshaw. "Potential Benefits, Limitations, and Harms of Clinical Guidelines." British Medican Journal, 1999: 527-530. 
APPENDICES 


\title{
Security Assessment Report Medirank
}

\author{
EDITORS \\ AUSTIN WHIPPLE \\ 20 NOV. 2012
}

DOCUMENT VERSION 1.0

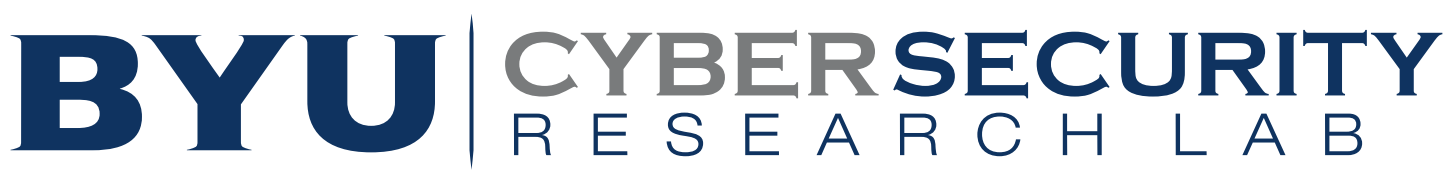

INFORMATION TECHNOLOGY, SCHOOL OF TECHNOLOGY

IRA A FULTON COLLEGE OF ENGINEERING AND TECHNOLOGY 


\section{A.1 Introduction}

\section{A.1.1 Executive Summary}

This report details the findings and recommendations of the BYU Cyber Security Research Lab Red Team during a security assessment of Medirank's virtual machine.

Information was gathered using port scans, vulnerability scans, and web browsing. With this information, exploits were attempted which include SQL injection, XSS injection, URL manipulation, and malicious file upload. We found that the web application and general system design had been done with security in mind, as we were unable to exploit anything. Medirank should be commended for the countermeasures in place but should remain vigilant in continued development to ensure that any potential future attack is not successful.

\section{A.1.2 Detailed Summary}

Reconnaissance was performed using a number of tools, such as nmap, Nikto, and OWASP Zap. The output of these tools is included in the report. It should be noted that some of the results were false positives, and were checked with manual testing or sqlmap to ensure there was no real vulnerability.

The web application uses cookies to enforce authentication. These cookies are currently not set to HTTP only which means they can be accessed by JavaScript. Allowing them to be accessed by JavaScript is almost never necessary, so setting them to HTTP-Only reduce the risk of cookie theft from malicious JavaScript.

Two directories, /scripts/ and /styles/ were found to have directory browsing enabled. While these two directories did not have any sensitive information, it is simpler to just have a global deny rule in an .htaccess file. 


\section{A.2 Testing}

The testing environment consisted of one Alienware laptop computer running both Windows 7 SP1 and Backtrack 5R1 in a dual boot configuration

The testing consisted of:

- Mapping the web application space of discovered websites.

- Intensive port-scan of live hosts to identify services.

- Vulnerability and service fingerprinting scans to identify versions, potential vulnerabilities, and operating systems.

- Booting the machine in single-user mode to perform source-code auditing

- Vulnerability scanning of web applications to identify potential attack vectors.

- Attempted Exploitation of services and web applications to confirm the existence and ascertain the risk of vulnerabilities

- Attempted malicious file upload

\section{A.3 Findings}

\section{A.3.1 Medirank}

Our finding was that the system was very robustly built. Use of SSH-keys for authentication removed the possibility of brute forcing SSH access. Using parameterized SQL queries prevented SQL injection, and htmlentities prevented XSS attacks.

Additionally, the machine only does exactly what it was built to do, and nothing more. Because there is very little development on the machine, and no scope creep, there were no unnecessary web applications or programs that provided us attack surface. 
Multiple tests were performed on the machine, using a combination of manual techniques and automated tools. One test in particular was that of uploading pictures. We attempted to upload PHP scripts embedded in JPGs, to have the server execute the PHP. However, the server did not accept our malicious images.

\section{A.4 Recommendations}

\section{A.4.1 Web Practices}

The two websites analyzed were susceptible to cookie forgeries. To help secure the website, cookies should be set to HTTP-Only. This is an additional flag set in the cookie HTTP response header that helps mitigate client side risk of accessing protected cookies. It indicates that the cookie should not be accessible on the client. A cross side scripting attack would fail to reveal cookie data with this practice. 


\section{APPENDIX B. SCAN RESULTS}

\section{B.1 Nmap}

\# Nmap 6.01 scan initiated Fri Nov 23 18:26:41 2012 as: nmap -sV -O2 -A -T5 -oN nmap_results.txt 192.168.42.178

Nmap scan report for 192.168.42.178

Host is up (0.00019s latency).

Not shown: 996 closed ports

PORT STATE SERVICE VERSION

22/tcp open ssh OpenSSH 5.9p1 Debian 5ubuntu1 (protocol 2.0)

| ssh-hostkey: 1024 90:73:c0:b2:68:99:9e:72:03:be:e4:29:59:2e:58:12 (DSA)

|_2048 79:0f:33:cc:51:bc:5d:c0:06:26:de:4e:ef:5b:1e:83 (RSA)

25/tcp open smtp Postfix smtpd

| ssl-cert: Subject: commonName=ubuntu

| Not valid before: 2012-03-07 06:01:26

|_Not valid after: 2022-03-05 06:01:26

I_smtp-commands: ubuntu, PIPELINING, SIZE 10240000, VRFY, ETRN, STARTTLS, ENHANCEDSTATUSCODES, 8BITMIME, DSN,

80/tcp open http Apache httpd

L_http-methods: No Allow or Public header in OPTIONS response (status code 302) 
| http-title: 302 Found

|_Did not follow redirect to https://192.168.42.178/

443/tcp open ssl/http Apache httpd

I_http-methods: No Allow or Public header in OPTIONS response (status code 200)

L_http-title: Medirank

| ssl-cert: Subject: commonName=medirank.it.et.byu.edu/organizationName=Medirank Research/stateOrProvinceName $=\mathrm{Utah} /$ countryName $=\mathrm{US}$

| Not valid before: 2012-11-01 16:21:10

I_Not valid after: 2013-11-01 16:21:10

MAC Address: 00:0C:29:8A:4E:99 (VMware)

Device type: general purpose|WAP|media device|webcam|storage-misc|phone|specialized Running (JUST GUESSING): Linux 2.6.X|3.X|2.4.X (97\%), Netgear embedded (96\%), Western Digital embedded (96\%), AXIS Linux 2.6.X (94\%), HP embedded (90\%), Crestron 2Series $(89 \%)$

OS CPE: cpe:/o:linux:kernel:2.6 cpe:/o:linux:kernel:3 cpe:/o:axis:linux:2.6 cpe:/o:linux:kernel:2.4.26 cpe:/o:google:android:2 cpe:/o:crestron:2_series

Aggressive OS guesses: Linux 2.6 .38 - 3.2 (97\%), Linux 3.0 - 3.1 (96\%), Netgear DG834G WAP or Western Digital WD TV media player (96\%), AXIS 210A or 211 Network Camera (Linux 2.6) (94\%), Linux 2.6.32 - 2.6.35 (94\%), Linux 2.6.38 - 3.0 (93\%), Linux 2.6.38 (93\%), Linux 2.6.17 - 2.6.36 (93\%), Linux 2.6.19 - 2.6.35 (93\%), Linux 2.6.36 - 2.6.37 (93\%)

No exact OS matches for host (test conditions non-ideal).

Network Distance: 1 hop

Service Info: Host: ubuntu; OS: Linux; CPE: cpe:/o:linux:kernel 
TRACEROUTE

HOP RTT ADDRESS

$1 \quad 0.19 \mathrm{~ms} 192.168 .42 .178$

OS and Service detection performed. Please report any incorrect results at http://nmap.org/submit/ .

\# Nmap done at Fri Nov 23 18:26:57 2012 -- 1 IP address (1 host up) scanned in 16.06 seconds

\section{B.2 Nikto}

- Nikto v2.1.5/2.1.5

+ Target Host: 192.168.42.178

+ Target Port: 443

+ GET /: Cookie PHPSESSID created without the secure flag

+ GET /: Cookie PHPSESSID created without the httponly flag

+ GET /: The anti-clickjacking X-Frame-Options header is not present.

+ -3268: GET /scripts/: /scripts/: Directory indexing found.

+ GET /: Hostname '192.168.42.178' does not match certificate's CN

'medirank.it.et.byu.edu/emailAddress=ccteng@byu.edu'

+ GET /images: IP address found in the 'location' header. The IP is "127.0.1.1".

+ -630: GET /images: IIS may reveal its internal or real IP in the Location header via a request to the /images directory. The value is "https://127.0.1.1/images/". 
+ DEBUG HASH(0x24ce500): DEBUG HTTP verb may show server debugging information. See http://msdn.microsoft.com/en-us/library/e8z01xdh\%28VS.80\%29.aspx for details.

+ -3092: GET /connect/: /connect/: This might be interesting...

+ -3268: GET /images/?pattern=/etc/*\&sort=name: /images/?pattern=/etc/*\&sort=name:

Directory indexing found.

+ GET /icons/README: Server leaks inodes via ETags, header found with file /icons/README, inode: 814356 , size: 5108, mtime: 0x438c0358aae80

+ -3233: GET /icons/README: /icons/README: Apache default file found. 


\section{APPENDIX C. USER SURVEY}

\section{Initial Report}

Last Modified: 11/15/2012

1. I felt the instructions adequately told me what I needed to do.

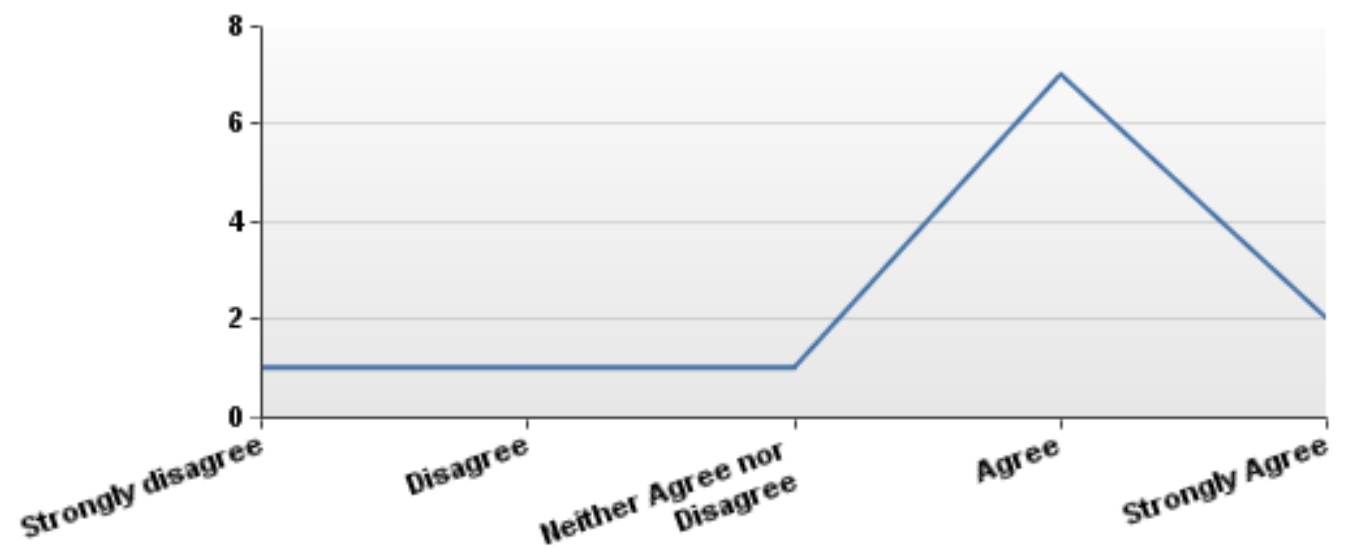

\begin{tabular}{|c|c|c|c|}
\hline$\#$ & Answer & Response & $\%$ \\
\hline 1 & $\begin{array}{l}\text { Strongly } \\
\text { disagree }\end{array}$ & 1 & $8 \%$ \\
\hline 2 & Disagree & 1 & $8 \%$ \\
\hline 3 & $\begin{array}{l}\text { Neither Agree } \\
\text { nor Disagree }\end{array}$ & 1 & $8 \%$ \\
\hline 4 & Agree & 7 & $58 \%$ \\
\hline \multirow[t]{2}{*}{5} & Strongly Agree & 2 & $17 \%$ \\
\hline & Total & 12 & $100 \%$ \\
\hline
\end{tabular}




\begin{tabular}{|l|r|}
\hline Statistic & Value \\
\hline Min Value & 1 \\
Max Value & 5 \\
Mean & 3.67 \\
Variance & 1.33 \\
Standard Deviation & 1.15 \\
Total Responses & 12 \\
\hline
\end{tabular}

\section{Dragging and dropping the images to rank was intuitive and easy to understand.}

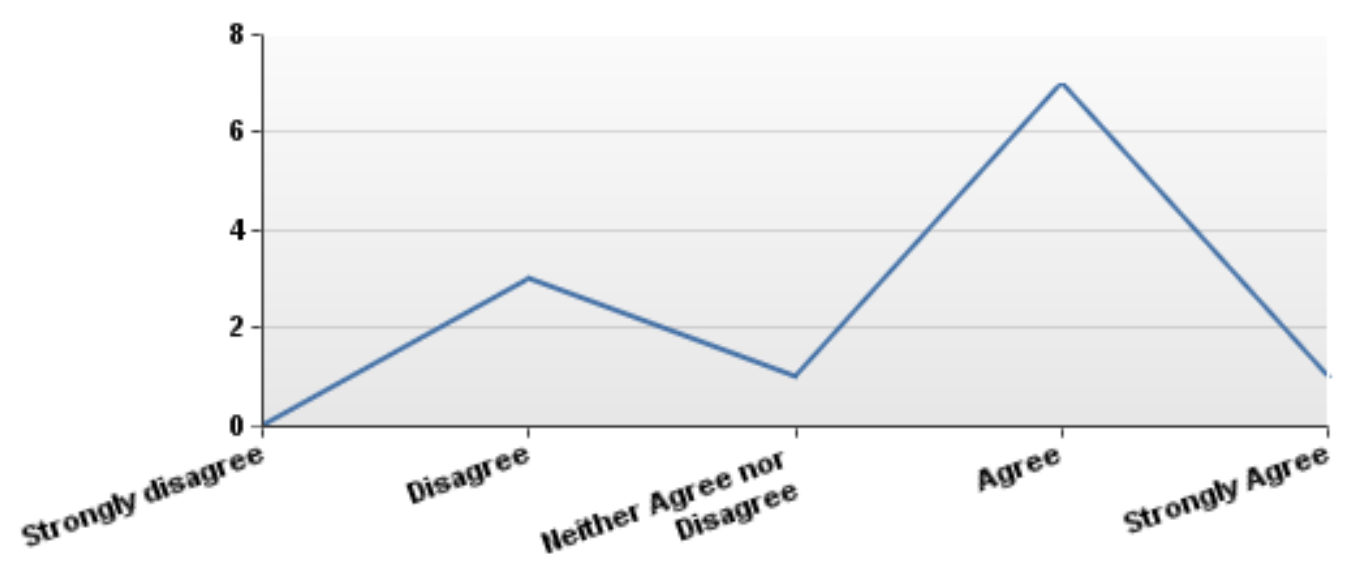

\begin{tabular}{|c|c|c|c|}
\hline$\#$ & Answer & Response & $\%$ \\
\hline 1 & Strongly disagree & 0 & $0 \%$ \\
\hline 2 & Disagree & 3 & $25 \%$ \\
\hline 3 & $\begin{array}{l}\text { Neither Agree nor } \\
\text { Disagree }\end{array}$ & 1 & $8 \%$ \\
\hline 4 & Agree & 7 & $58 \%$ \\
\hline \multirow[t]{2}{*}{5} & Strongly Agree & 1 & $8 \%$ \\
\hline & Total & 12 & $100 \%$ \\
\hline
\end{tabular}




\begin{tabular}{|l|r|}
\hline Statistic & Value \\
\hline Min Value & 2 \\
Max Value & 5 \\
Mean & 3.50 \\
Variance & 1.00 \\
Standard Deviation & 1.00 \\
Total Responses & 12 \\
\hline
\end{tabular}

\section{The size of the images made it difficult to properly rank.}

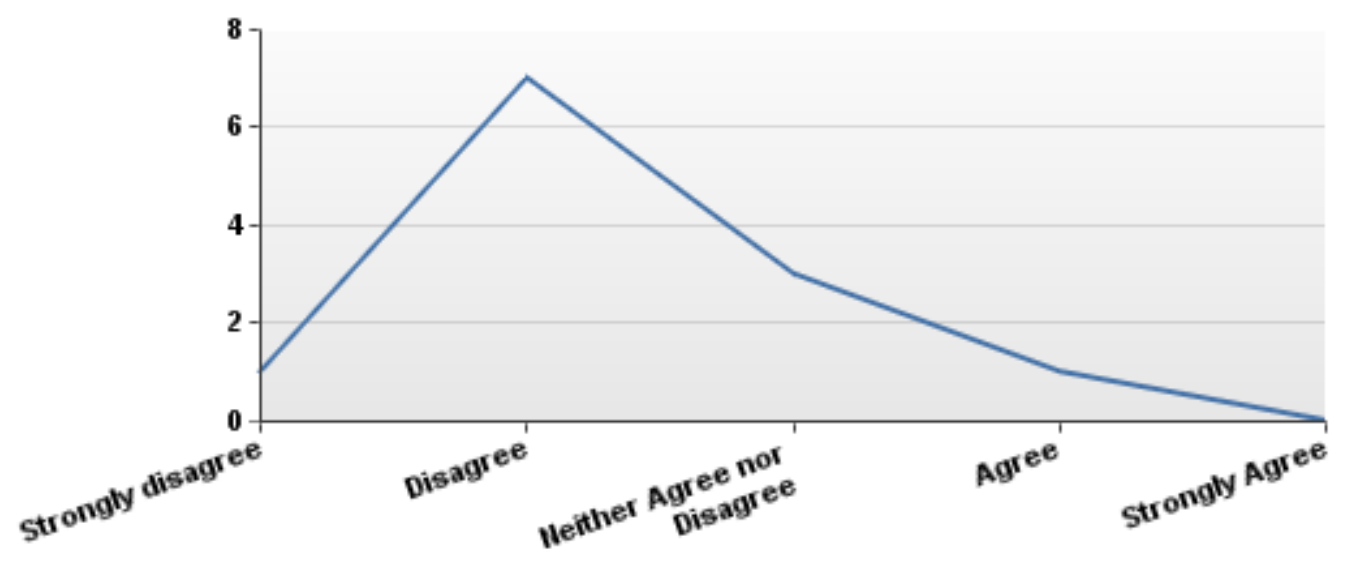

\begin{tabular}{|c|l|c|c|}
\hline$\#$ & Answer & Response & $\%$ \\
\hline 1 & Strongly disagree & 1 & $8 \%$ \\
2 & Disagree & 7 & $58 \%$ \\
3 & Neither Agree nor Disagree & 3 & $25 \%$ \\
4 & Agree & 1 & $8 \%$ \\
5 & Strongly Agree & 0 & $0 \%$ \\
\hline & Total & 12 & $100 \%$ \\
\hline
\end{tabular}




\begin{tabular}{|l|r|}
\hline Statistic & Value \\
\hline Min Value & 1 \\
\hline Max Value & 4 \\
Mean & 2.33 \\
Variance & 0.61 \\
Standard Deviation & 0.78 \\
\hline Total Responses & 12 \\
\hline
\end{tabular}

\section{The adjectives, ie "most severe" and "least severe", helped me understand how to order the images.}

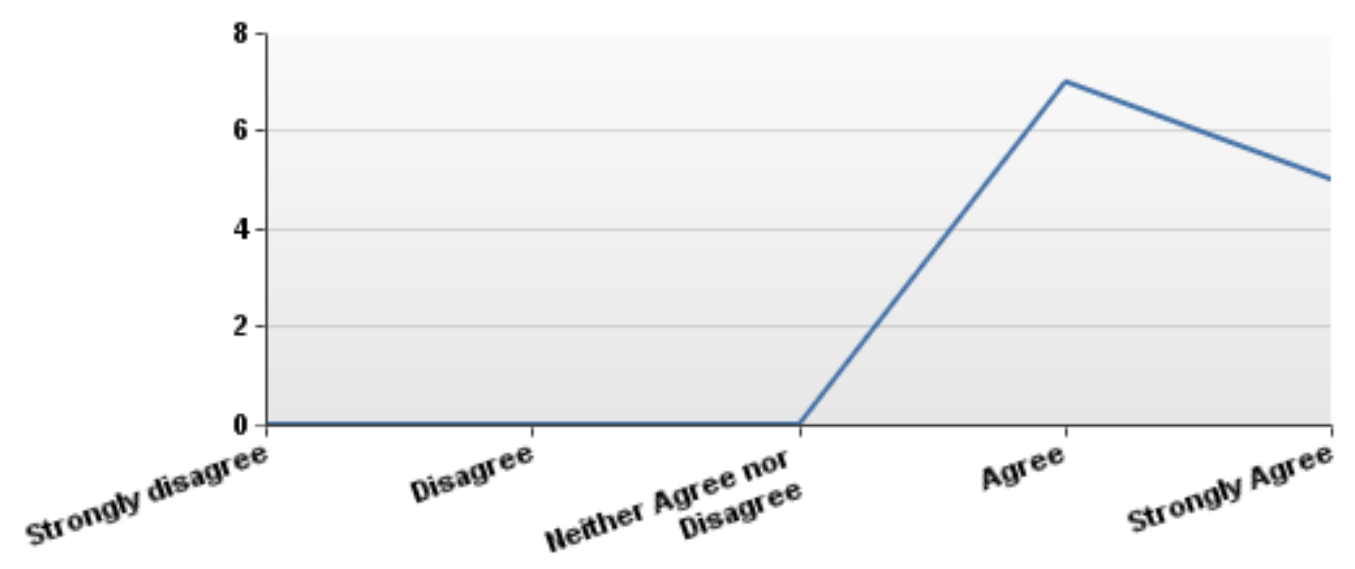

\begin{tabular}{|c|l|c|c|c|}
\hline$\#$ & Answer & & Response & $\%$ \\
\hline 1 & Strongly disagree & & 0 & $0 \%$ \\
2 & Disagree & 0 & $0 \%$ \\
3 & Neither Agree nor Disagree & & 0 & $0 \%$ \\
4 & Agree & & 7 & $58 \%$ \\
5 & Strongly Agree & & 5 & $42 \%$ \\
\hline & Total & 12 & $100 \%$ \\
\hline
\end{tabular}




\begin{tabular}{|l|r|}
\hline Statistic & Value \\
\hline Min Value & 4 \\
Max Value & 5 \\
Mean & 4.42 \\
Variance & 0.27 \\
Standard Deviation & 0.51 \\
Total Responses & 12 \\
\hline
\end{tabular}

\section{I tried to save my place.}

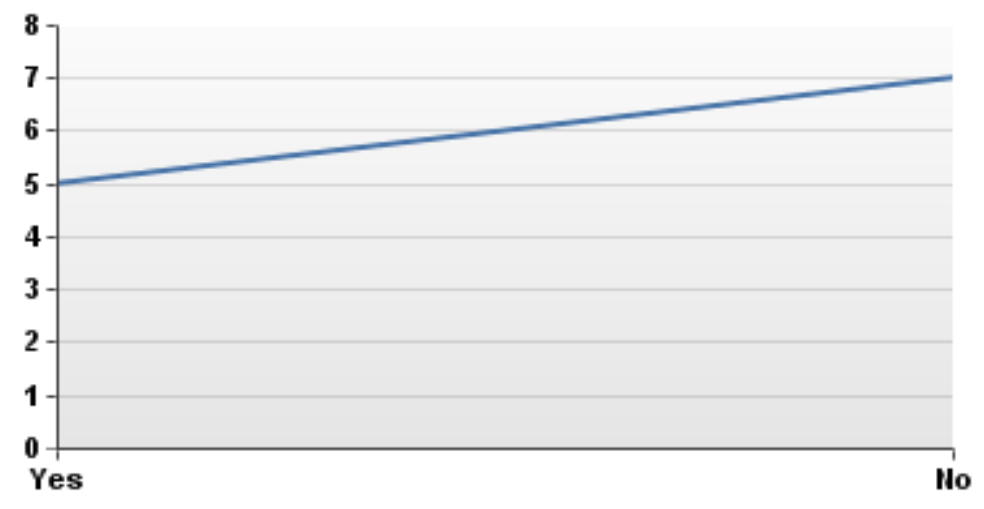

\begin{tabular}{|c|l|c|c|c|}
\hline$\#$ & Answer & Response & $\%$ \\
\hline 1 & Yes & 5 & $42 \%$ \\
2 & No & 7 & $58 \%$ \\
\hline & Total & 12 & $100 \%$ \\
\hline
\end{tabular}

\begin{tabular}{|l|r|}
\hline Statistic & Value \\
\hline Min Value & 1 \\
Max Value & 2 \\
Mean & 1.58 \\
Variance & 0.27 \\
Standard Deviation & 0.51 \\
Total Responses & 12 \\
\hline
\end{tabular}




\section{If yes, Saving my progress worked as I expected.}

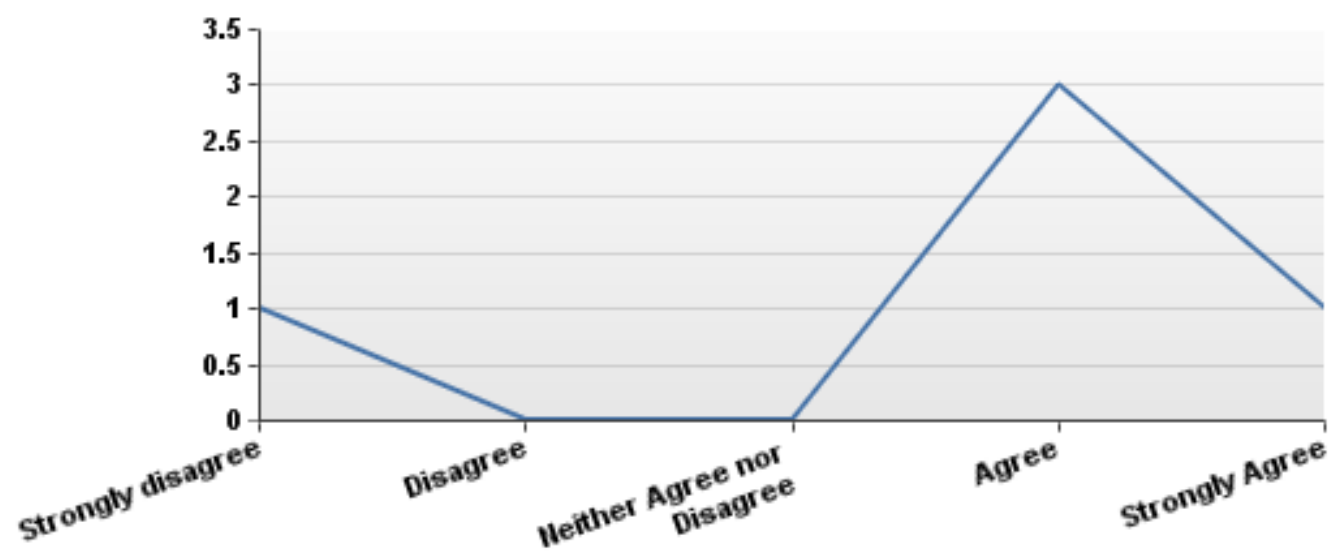

\begin{tabular}{|c|c|c|c|}
\hline$\#$ & Answer & Response & $\%$ \\
\hline 1 & Strongly disagree & 1 & $20 \%$ \\
\hline 2 & Disagree & 0 & $0 \%$ \\
\hline 3 & Neither Agree nor Disagree & 0 & $0 \%$ \\
\hline 4 & Agree & 3 & $60 \%$ \\
\hline \multirow[t]{2}{*}{5} & Strongly Agree & 1 & $20 \%$ \\
\hline & Total & 5 & $100 \%$ \\
\hline
\end{tabular}

\begin{tabular}{|l|r|}
\hline Statistic & Value \\
\hline Min Value & 1 \\
Max Value & 5 \\
Mean & 3.60 \\
Variance & 2.30 \\
Standard Deviation & 1.52 \\
\hline Total Responses & 5 \\
\hline
\end{tabular}




\section{I was satisfied with my experience.}

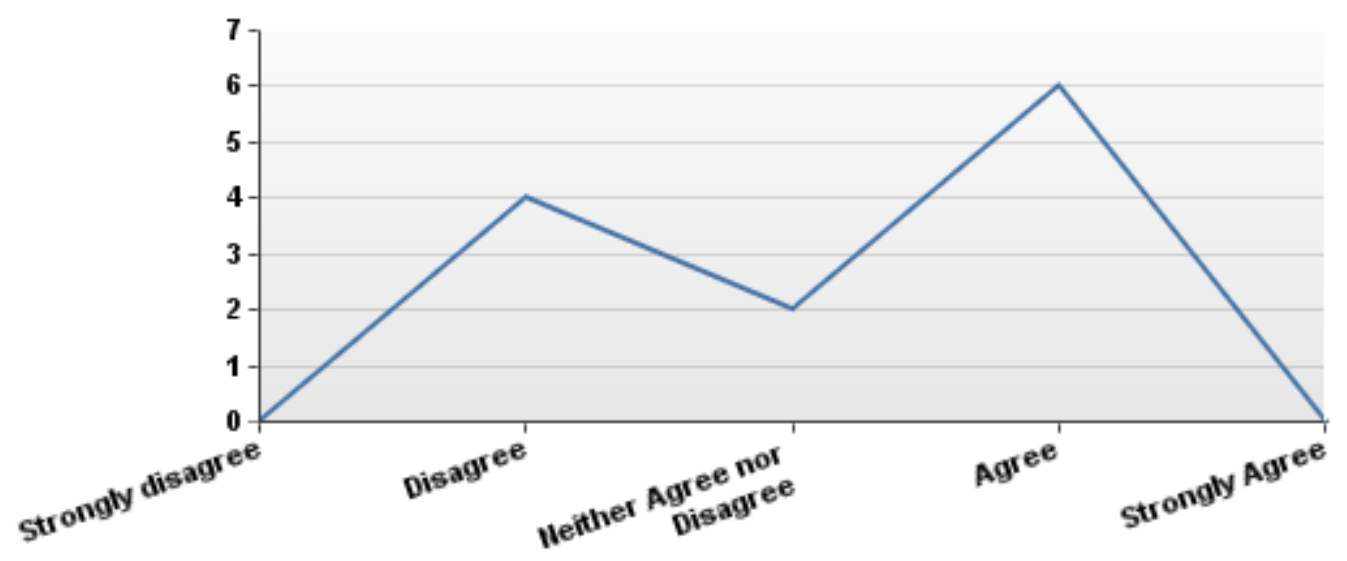

\begin{tabular}{|c|l|c|c|}
\hline$\#$ & Answer & Response & $\%$ \\
\hline 1 & Strongly disagree & 0 & $0 \%$ \\
2 & Disagree & 4 & $33 \%$ \\
3 & Neither Agree nor Disagree & 2 & $17 \%$ \\
4 & Agree & 6 & $50 \%$ \\
5 & Strongly Agree & 0 & $0 \%$ \\
\hline & Total & 12 & $100 \%$ \\
\hline
\end{tabular}

\begin{tabular}{|l|r|}
\hline Statistic & Value \\
\hline Min Value & 2 \\
Max Value & 4 \\
Mean & 3.17 \\
Variance & 0.88 \\
Standard Deviation & 0.94 \\
Total Responses & 12 \\
\hline
\end{tabular}




\section{Please share any other details of your experiences, good or bad.}

\section{Text Response}

Was too difficult to order with a long list on the right. Some images got frozen and would move anywhere.

The only thing I'd consider changing is the "vertical growth" as you start ranking pictures. It made it difficult to scroll up and down to compare the picure and the already ranked pictures.

I have more "real estate" horizontally than vertically. It would have been easier to rank seeing more images horizontally than the vertical setup that was used.

The instructions were inadequate. I first put them in order in the original block and did not understand to move them all over. Having one vertical and one horizontal was not easy to drag down to where you wanted them. It would have been better to have them both horizontal on the page so I wcould see them all at the same time.

The final set needs to scroll independently of the page so that you don't have to scroll the entire page down to drop an image at the end of your set, and then scroll all the way back up to see the rest of the images. Something like on those blogs that you hate - where the middle insert with text scrolls, and the backdrop remains stationary - might be an improvement. That way the images needing to be ranked stay put, but the bottom of your final set is more accessible. It got annoying having to scroll back to the top of the page over and over and over again.

Seems like they should have been sorted in place, versus dragging to the right. Or possible, have then all to the right and then drag on to a grid listing most severe to least severe. It worked, but I though initially I should sort in place. But then again, I did not read the instructions. :) Also, why so many items, I would rather sort 5 items, versus 50 items. So, 5 items 10 times versus 50 items one time.

Everything worked well once I realized that I needed to use Google Chrome instead of GoogleWindows Internet explorer.

The dragging and dropping of the images was clunky when the list started getting longer and you had to put images at the bottom.

For me it would have been easier to rank the pictures if I could have seen all of them at the same time. Having to scroll up and down to re-place each picture was a little tedious.

It was really difficult to rank so many images in a vertical layout. It was fine to begin with, but once you have to scroll it was a pain. If there were less images or another layout it would have made it a lot better. I'm not an expert but that seemed like a lot of images to rank. Half as much seems like it would still give valuable and useful data. The format and site was nice and useful. It was simple and easy enough to understand. It made sense what to do. It was just difficult to do it (comments above).

\begin{tabular}{|l|r|}
\hline Statistic & Value \\
\hline Total Responses & 10 \\
\hline
\end{tabular}




\section{APPENDIX D. SITE CODE}

\section{D.1 createStudy.php}

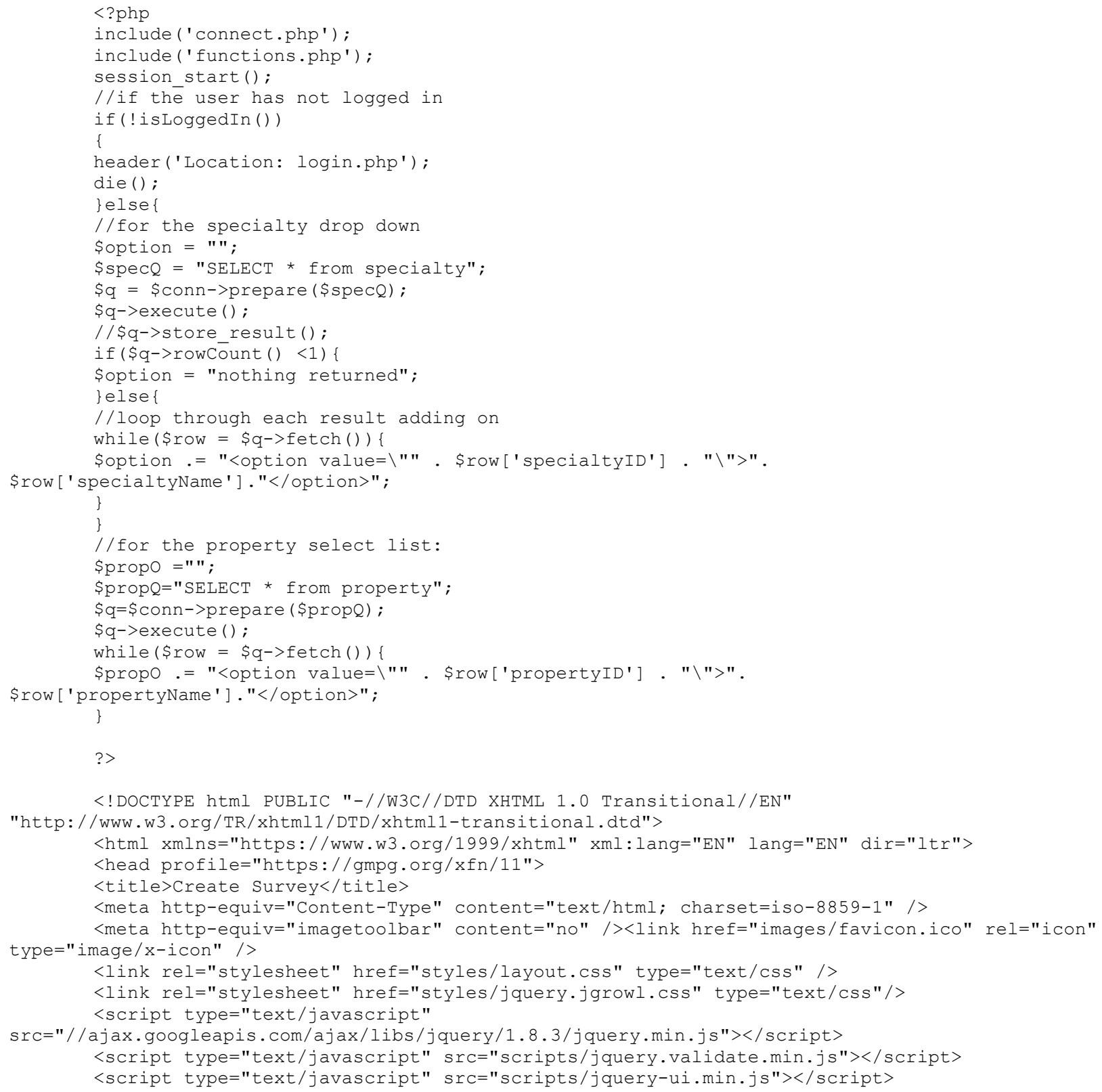




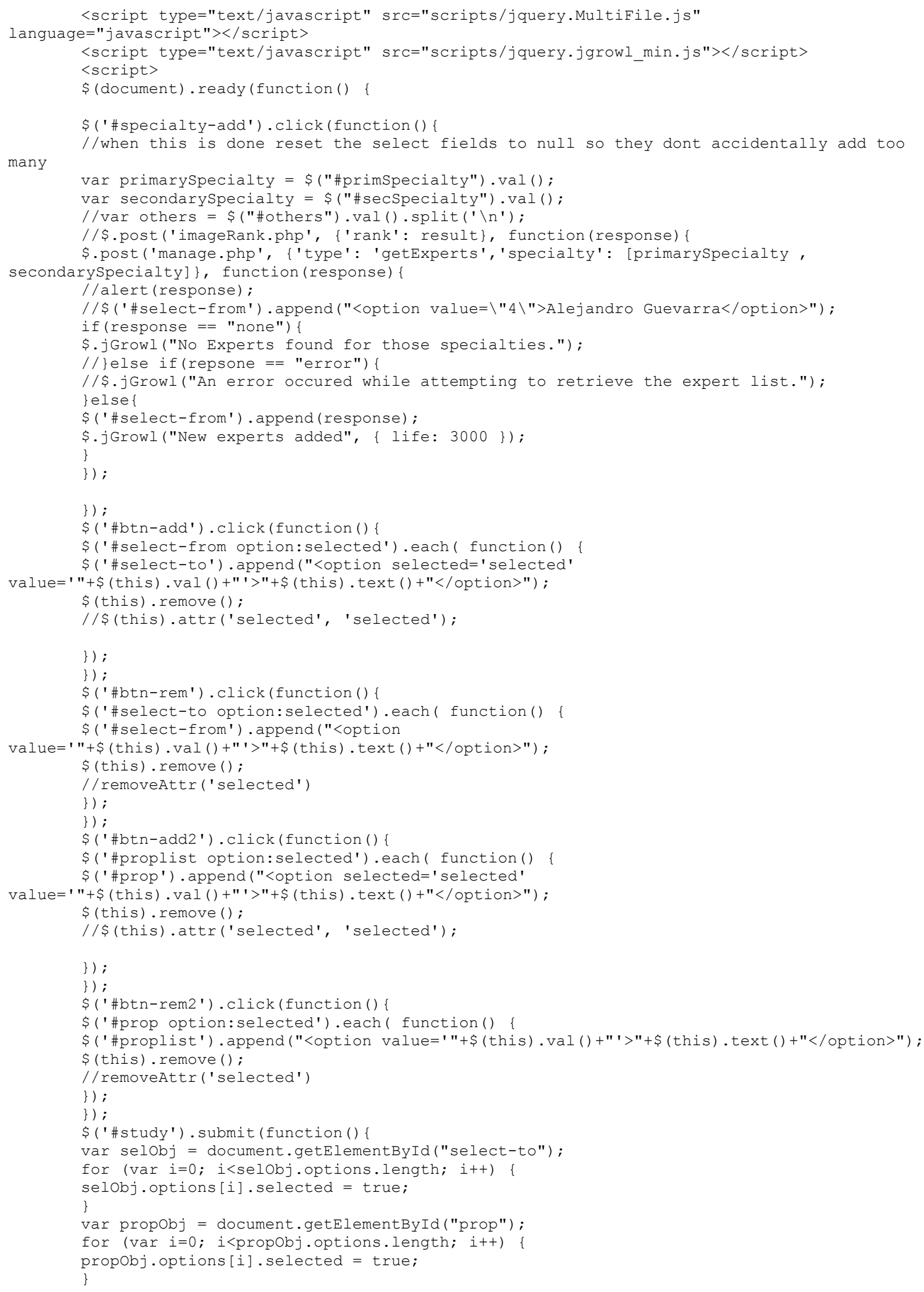




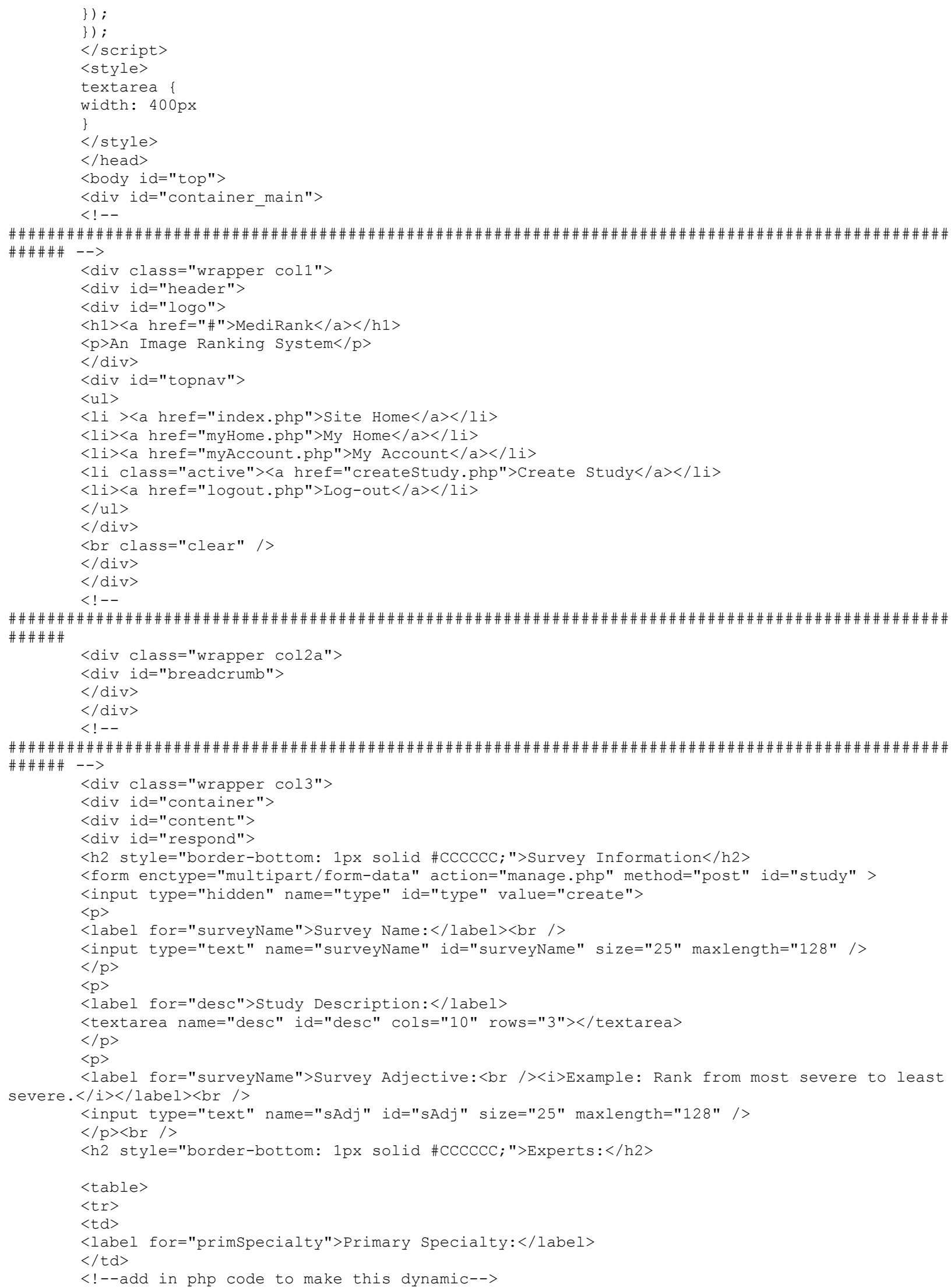




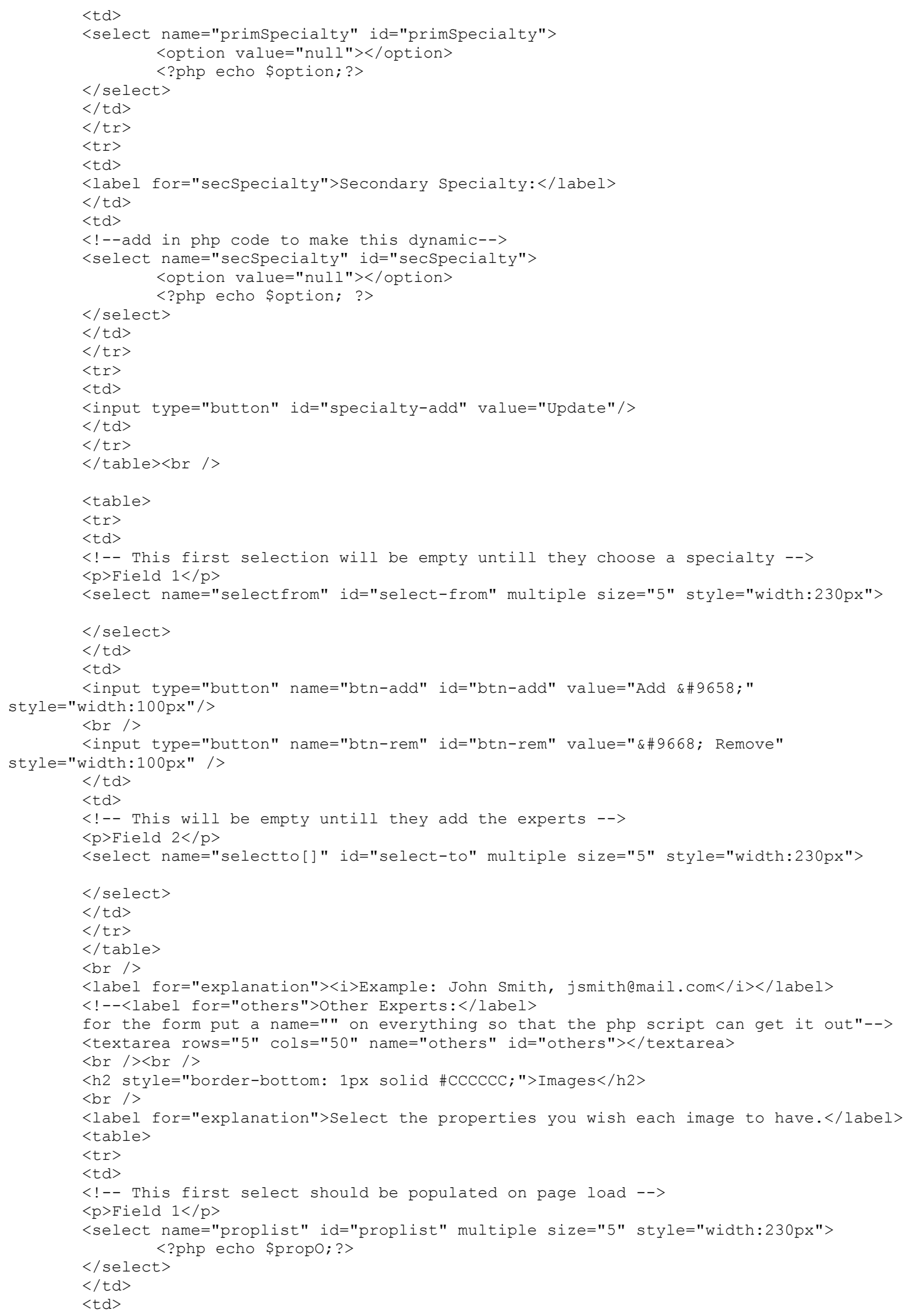




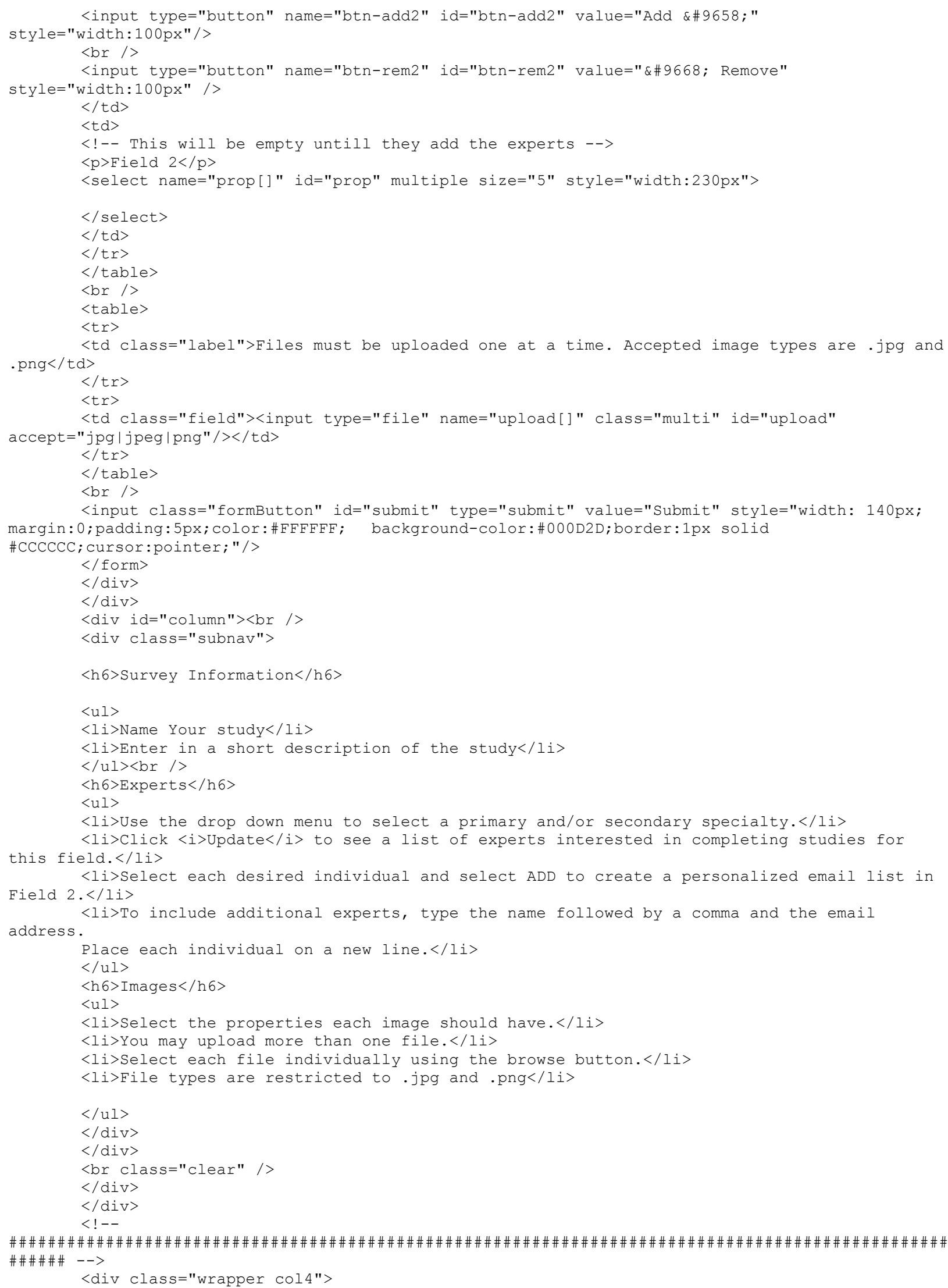




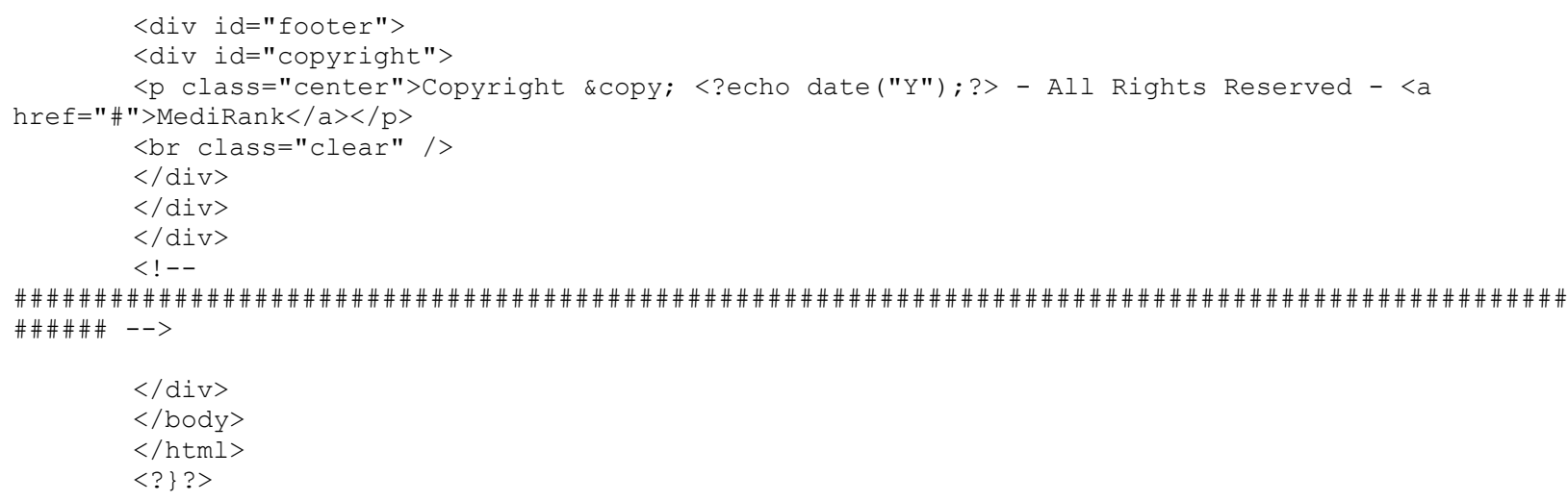

\section{D.2 manageStudies.php}

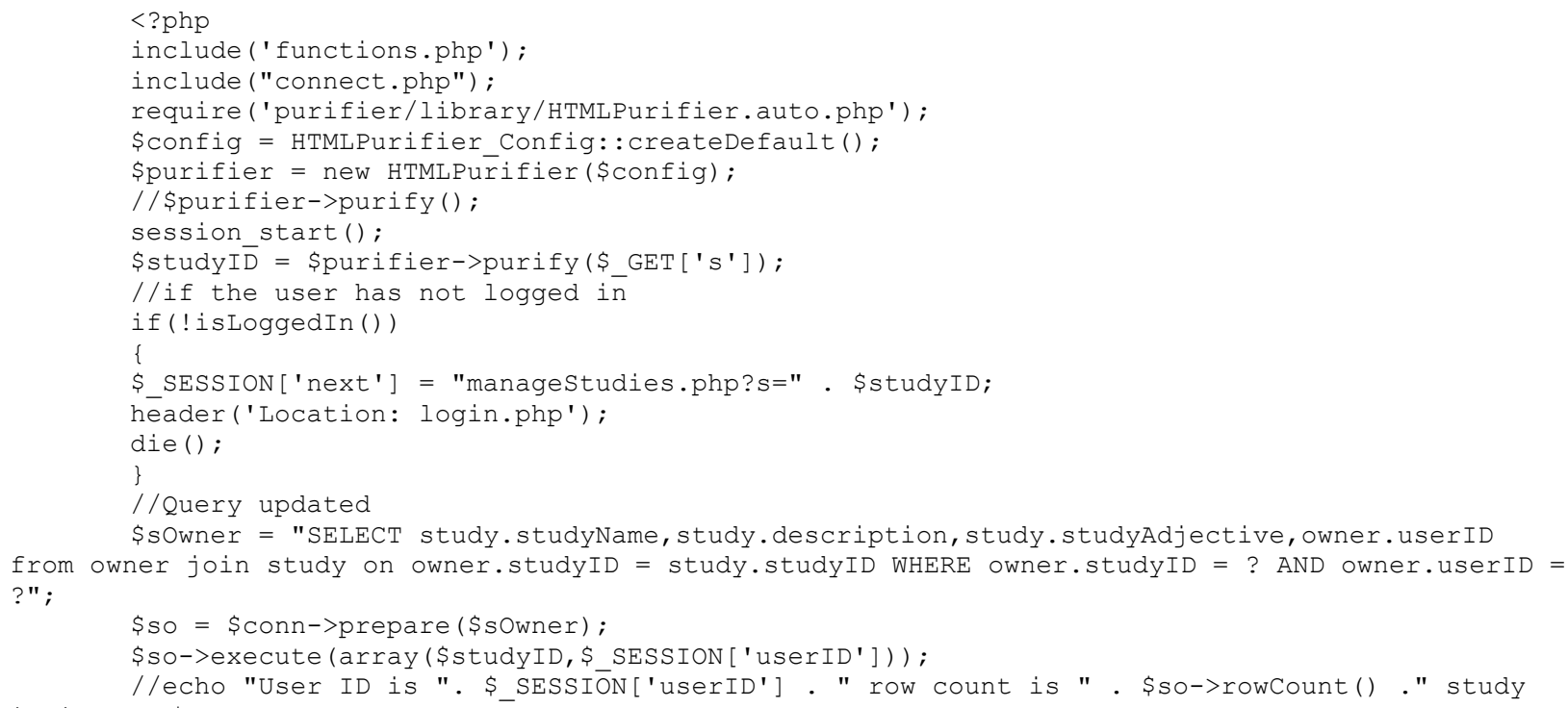




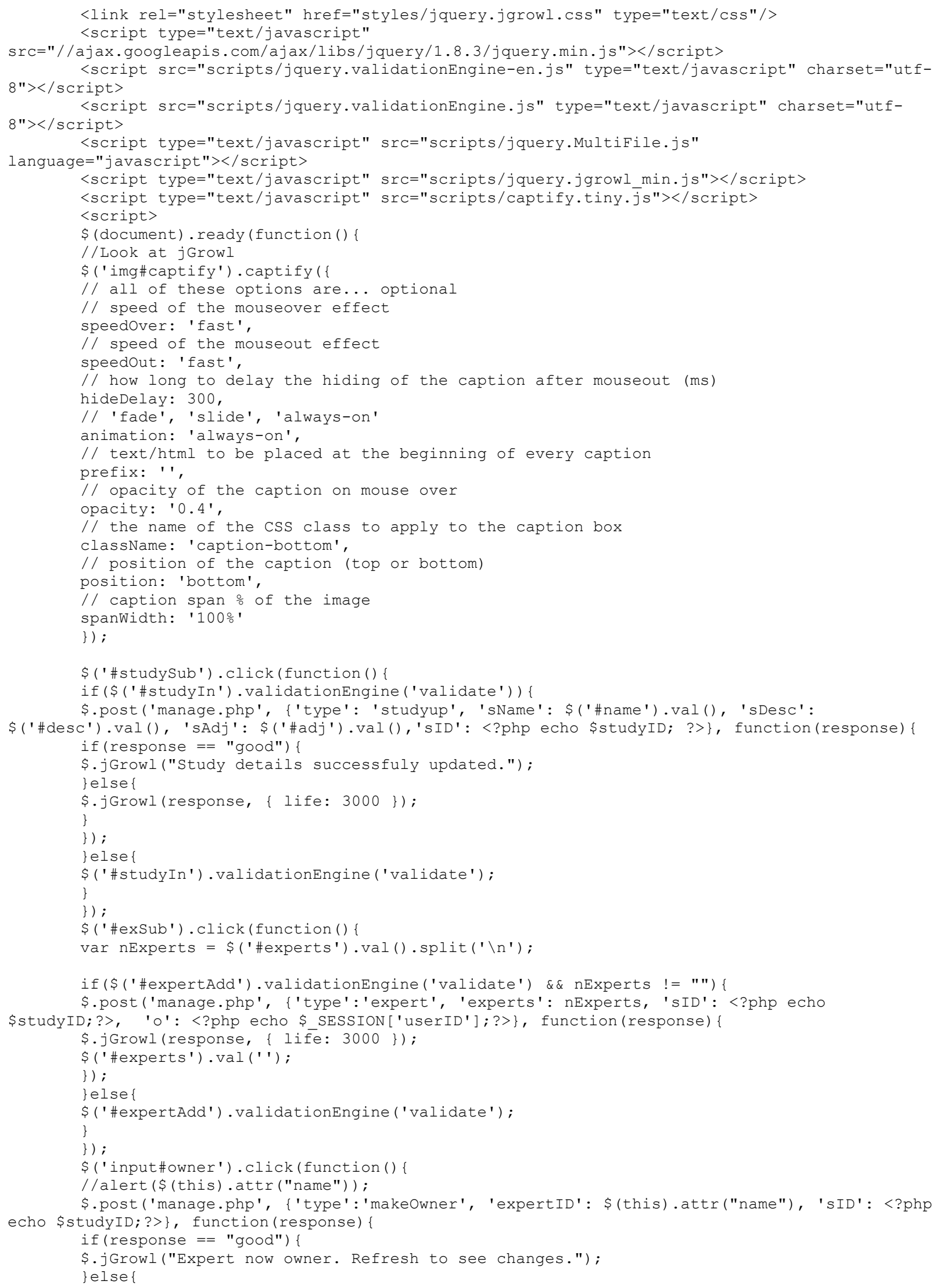




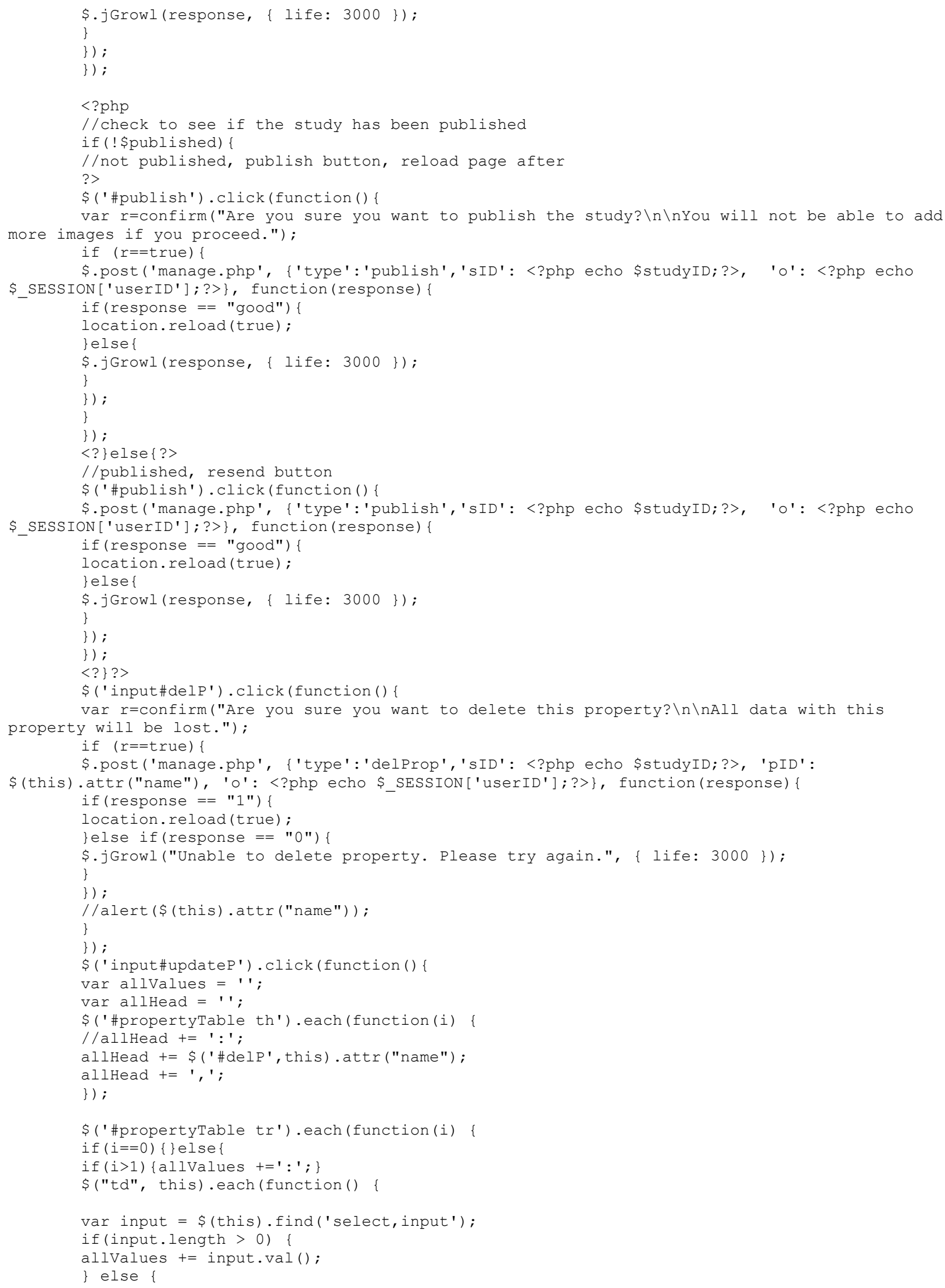




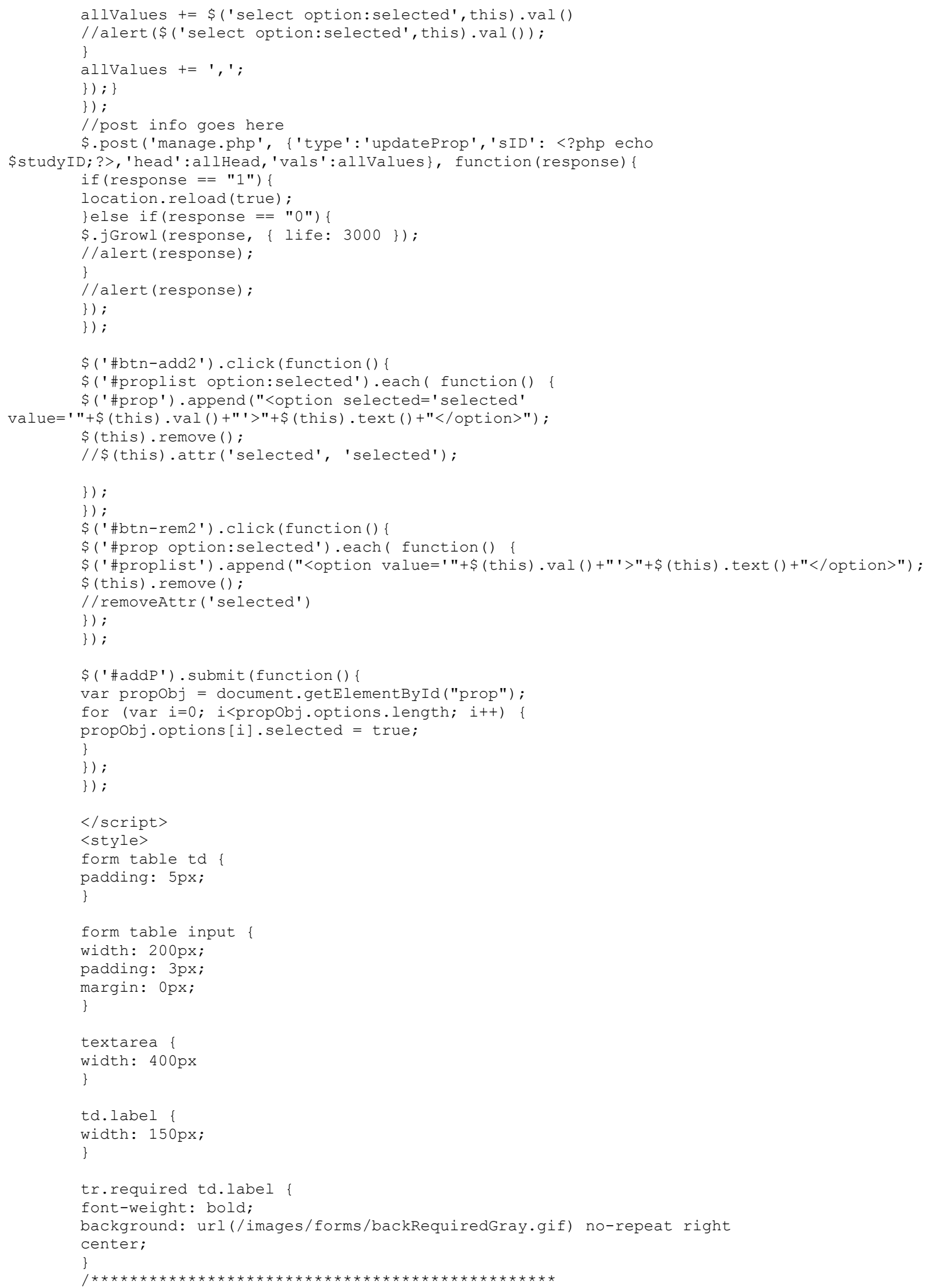




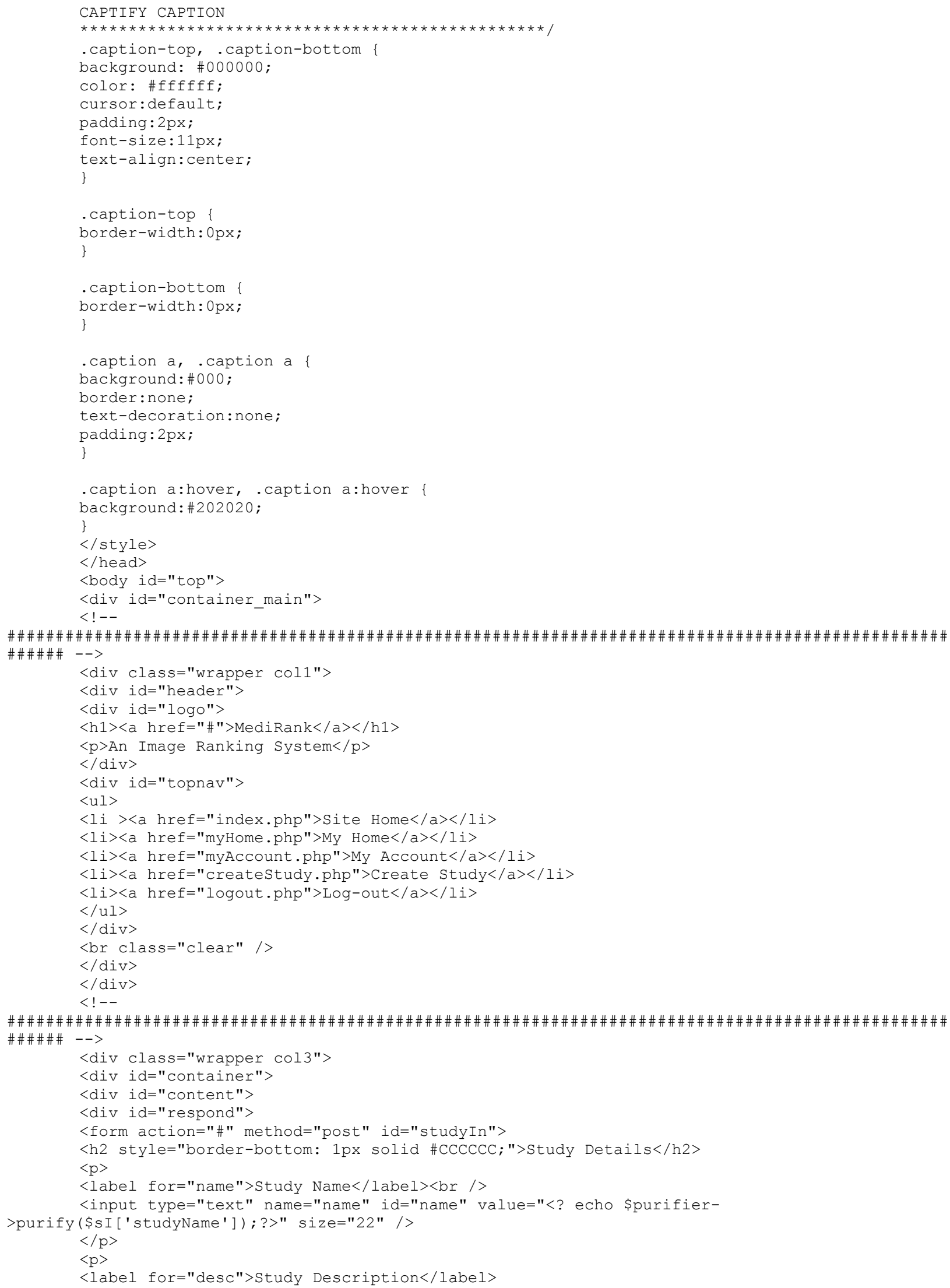


<textarea name="desc" id="desc" cols="10" rows="3"><? echo \$purifier-

$>$ purify (\$sI ['description']); ?></textarea $>$

$</ \mathrm{p}>$

$<\mathrm{p}>$

$<$ label for="name" $>$ Study Adjective $<$ br $/><i>$ Example: Rank from most severe to least severe. $</$ i $></$ label $><$ br $/>$

<input type="text" name="adj" id="adj" value="<? echo \$purifier-

>purify (\$sI['studyAdjective']) ; ?>" size="22" />

$</ \mathrm{p}>$

$<\mathrm{p}>$

<input name="studySub" type="button" id="studySub" value="Update study" />

$</ \mathrm{p}>$

$</$ form $>$

$<$ br $/><$ br $/>$

$<$ h2 style="border-bottom: 1px solid \#CCCCCC;" $>$ Publish study $</$ h2 $><$ p $>$

$<$ ?php

//check to see if the study has been published

if (! \$published) \{?>

$<\mathrm{p}>$

<input name="publish" type="button" id="publish" value="Publish Study" />

$</ \mathrm{p}>$

$<$ ? $\}$ else $\{/ /$ resend email? $>$

$<\mathrm{p}>$

<input name="publish" type="button" id="publish" value="Resend Email" />

$</ \mathrm{p}>$

$<$ ?

$?>$

$<\mathrm{br} /><\mathrm{br} />$

$<$ h2 style="border-bottom: 1px solid \#CCCCCC;">Current study Participants $</$ h2 $>$

<table cellpadding="0" cellspacing="0"

class="tablesorter" $><$ thead $>\langle$ tr $><$ th $>$ Name $</$ th $><$ th $>$ Email $</$ th $\rangle\langle$ th $>$ Complete $</$ th $><$ th $>$ Type $</$ th $></$ tr $></$ th ead $><$ tbody $>$

$<$ ?php

\$tableInfo = " ";

//get all owners

\$sOwners = "select

users.firstName, users.lastName, users.username, users.userID, count(results.resultID) as res from users left join owner on users.userID = owner.userID left join experts on experts.userID = owner.userID left join results on experts.expertID = results.expertID where owner.studyID $=$ ? group by users.userID";

\$sos $=$ \$conn->prepare (\$sOwners);

\$sos->execute (array (\$studyID));

$\$ i=0$;

while(\$owner $=$ \$sos->fetch ()$)\{$

if $(\$ i \div 2=0)\{$

\$class = "even";

\}else \{

\$class = "odd";

\}

\$tableInfo . = "<tr class=\"\$class \"><td $>"$. \$purifier->purify(\$owner['firstName'])." ". \$purifier->purify (\$owner['lastName']) . "</td $><t d>"$. \$purifier-

>purify (\$owner ['username']) . "</td $>$ ";

if (\$owner ['res'] > 0) \{

\$tableInfo .= "<td $>$ Rank Complete $</$ td $><t d>$ Owner $</$ td $\rangle</$ tr $\rangle$ " ;

\}else \{

\$tableInfo .= "<td $>$ Rank Incomplete $</$ td $><t d>$ Owner $</$ td $\rangle\langle/$ tr $\rangle$ ";

\}

$\$ i++$;

\}

/ /get all experts

\$sExperts $=$ "select

users.firstName, users.lastName, users.username, users.userID, count(results.resultID) as res from users left join experts on users.userID = experts.userID left join results on results.expertiD = experts.expertID where experts.userID not in (select userID from owner where studyID = ?) and experts.studyID $=$ ? group by users.userID";

\$ses $=\$$ conn->prepare $(\$ s E x p e r t s) ;$

\$ses->execute (array (\$studyID, \$studyID)) ;

while( Sexpert $=$ \$ses->fetch ()) \{ 


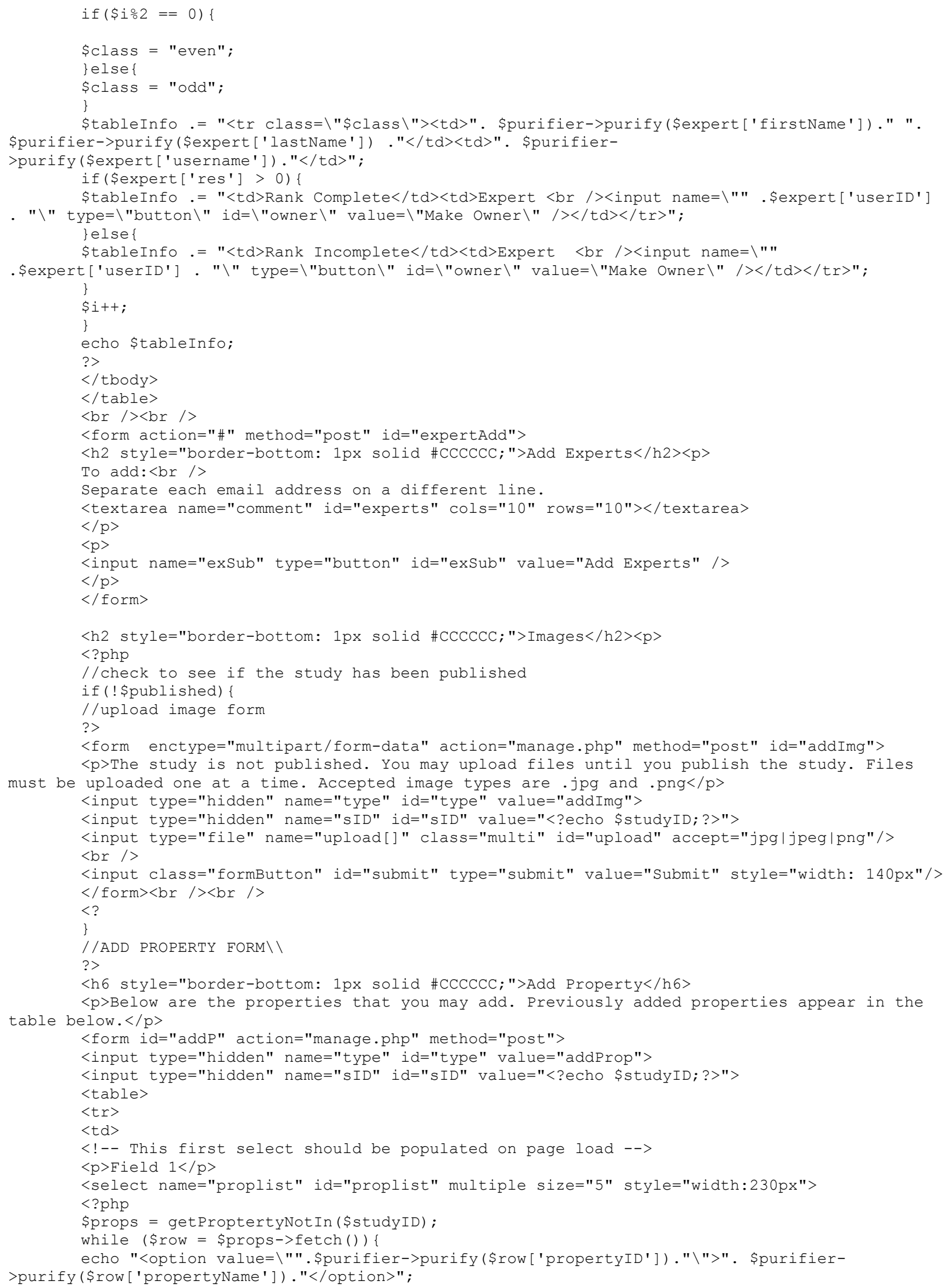




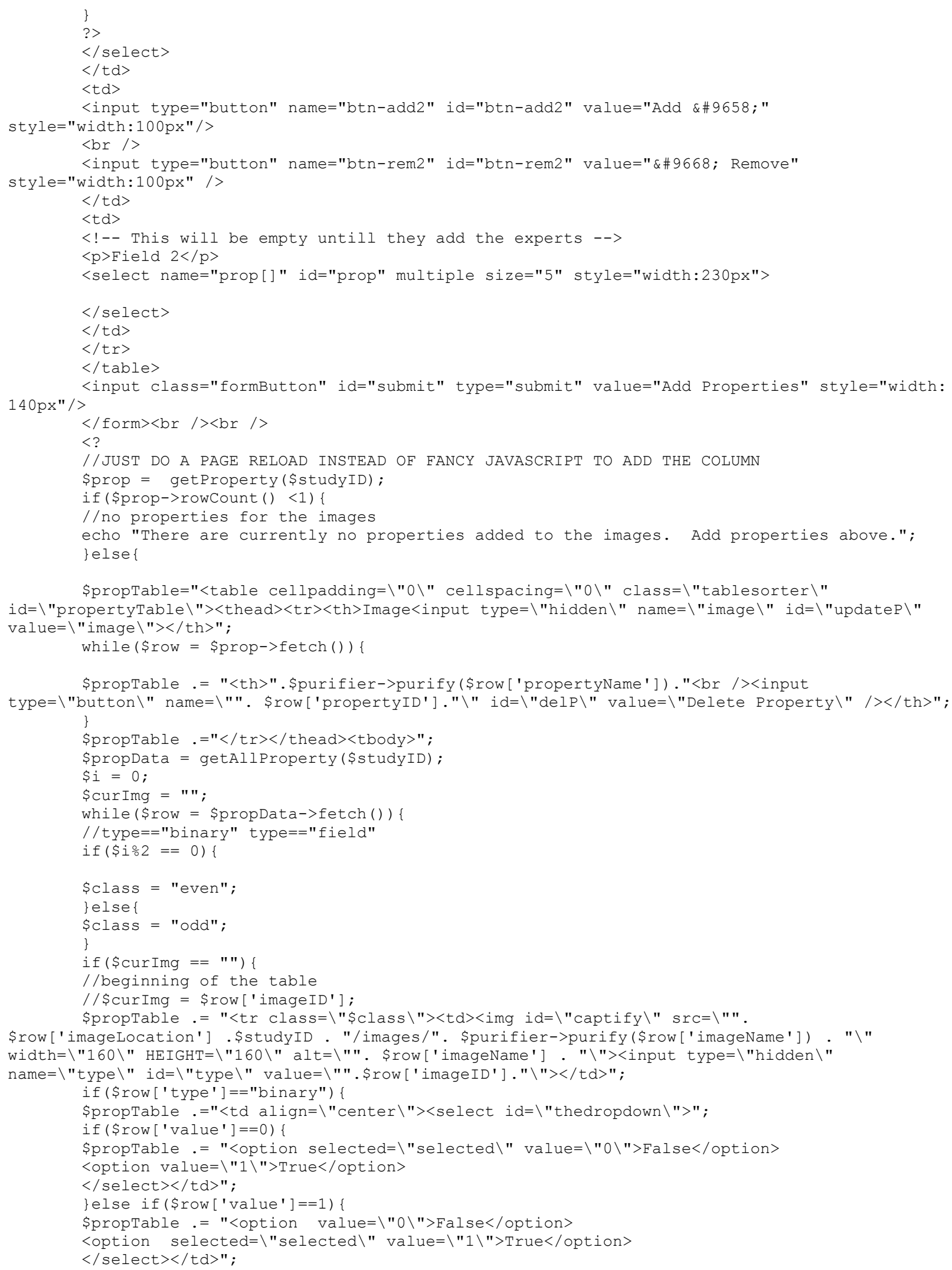




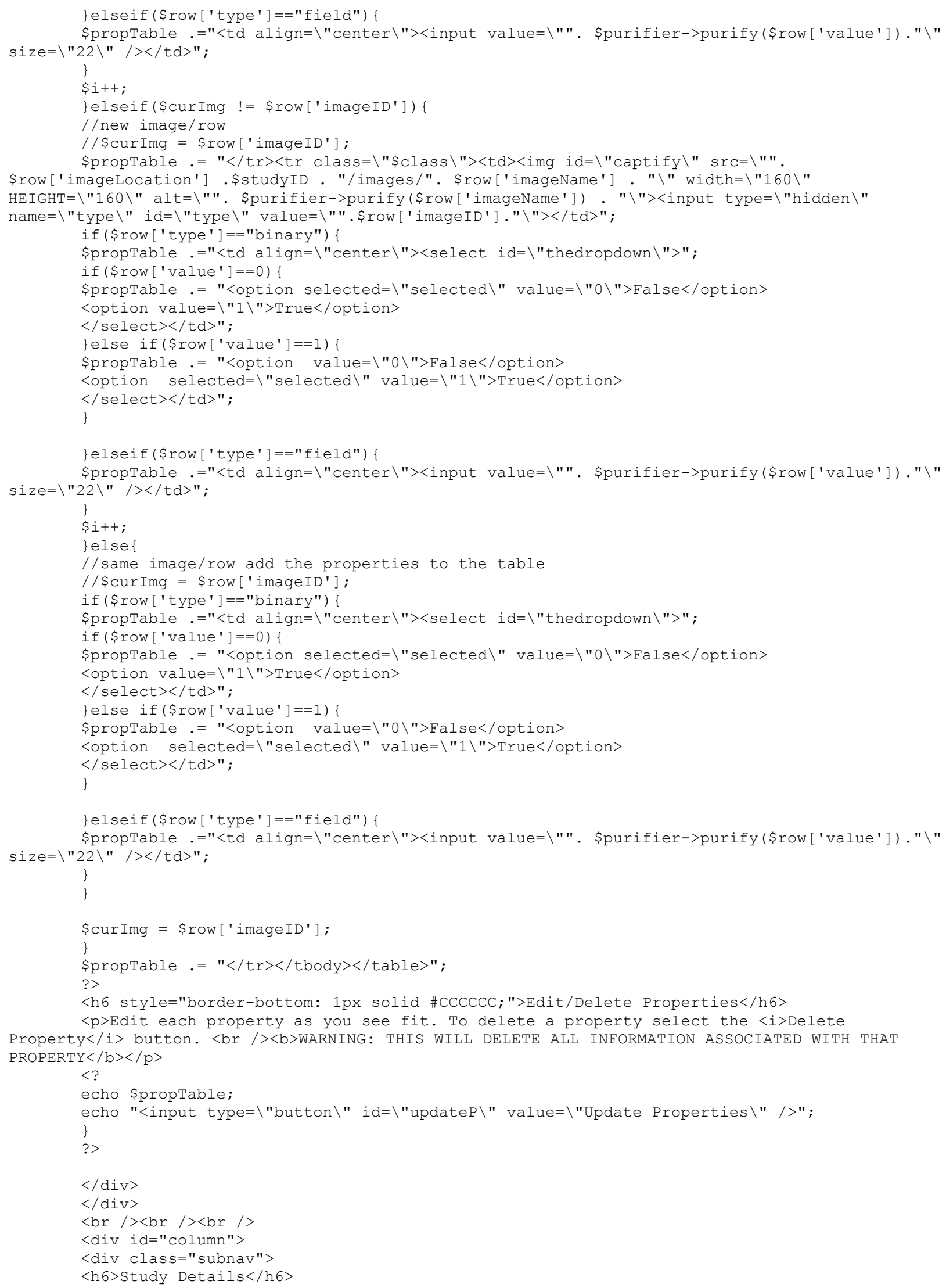




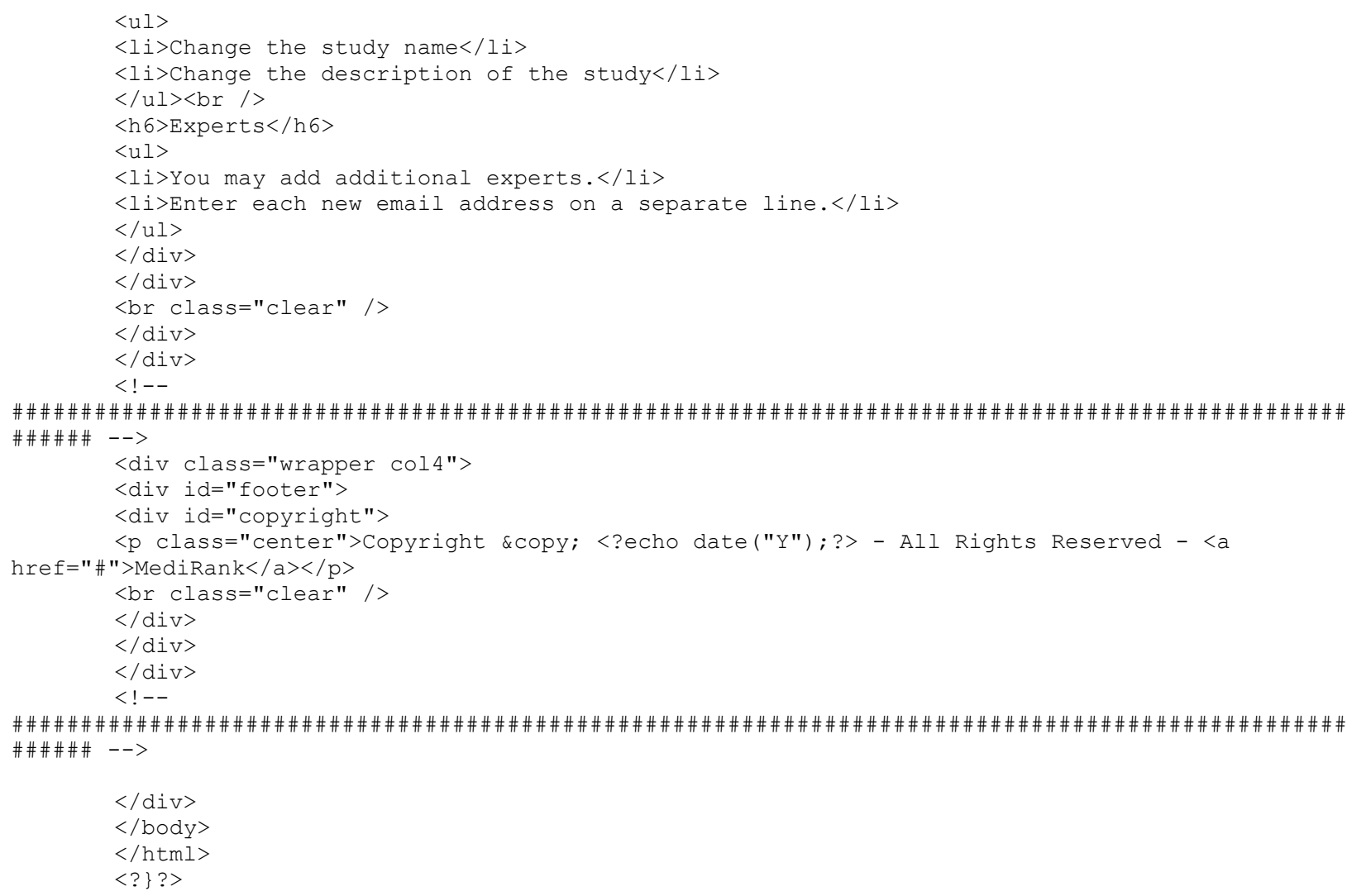

\section{D.3 rank.php}

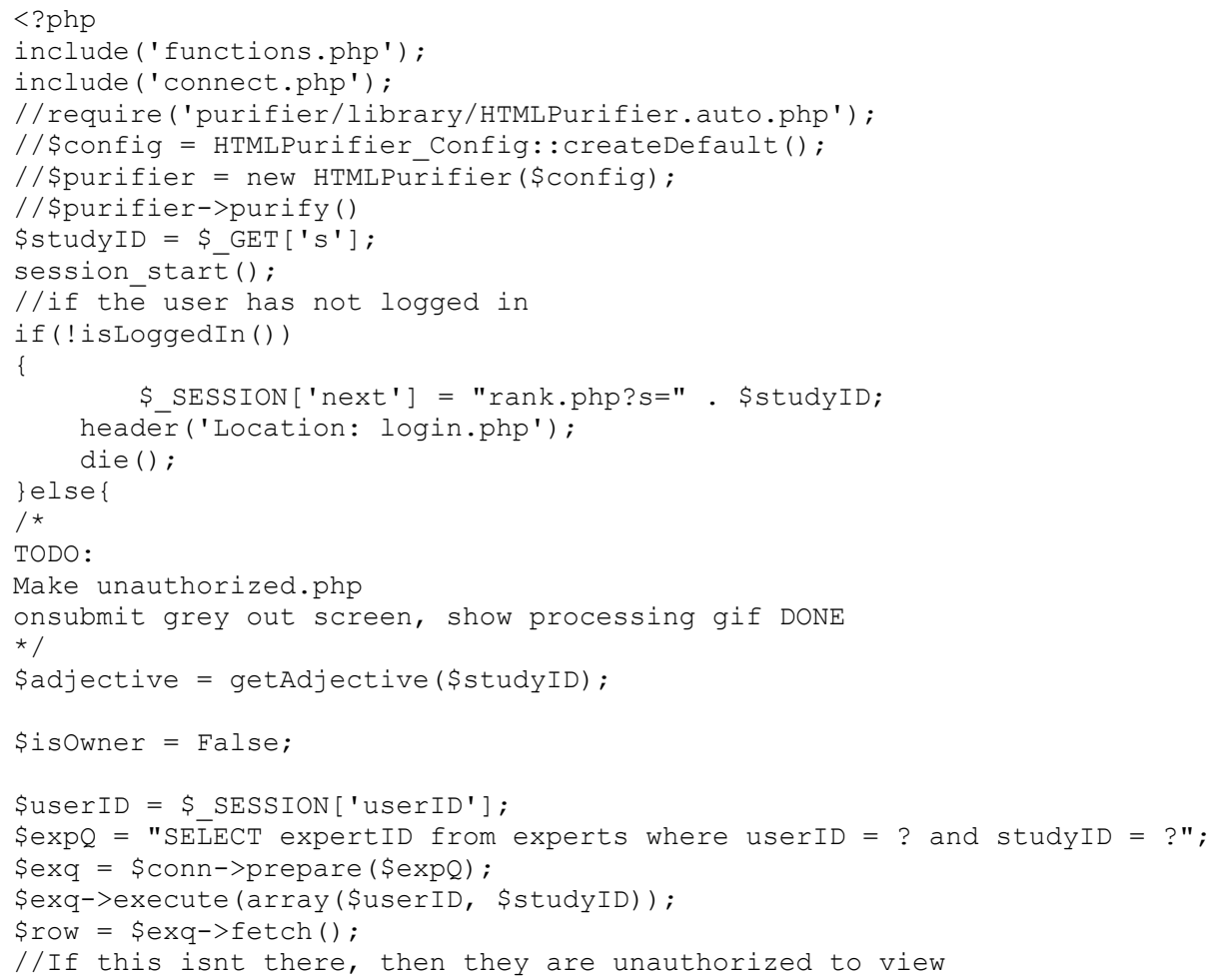




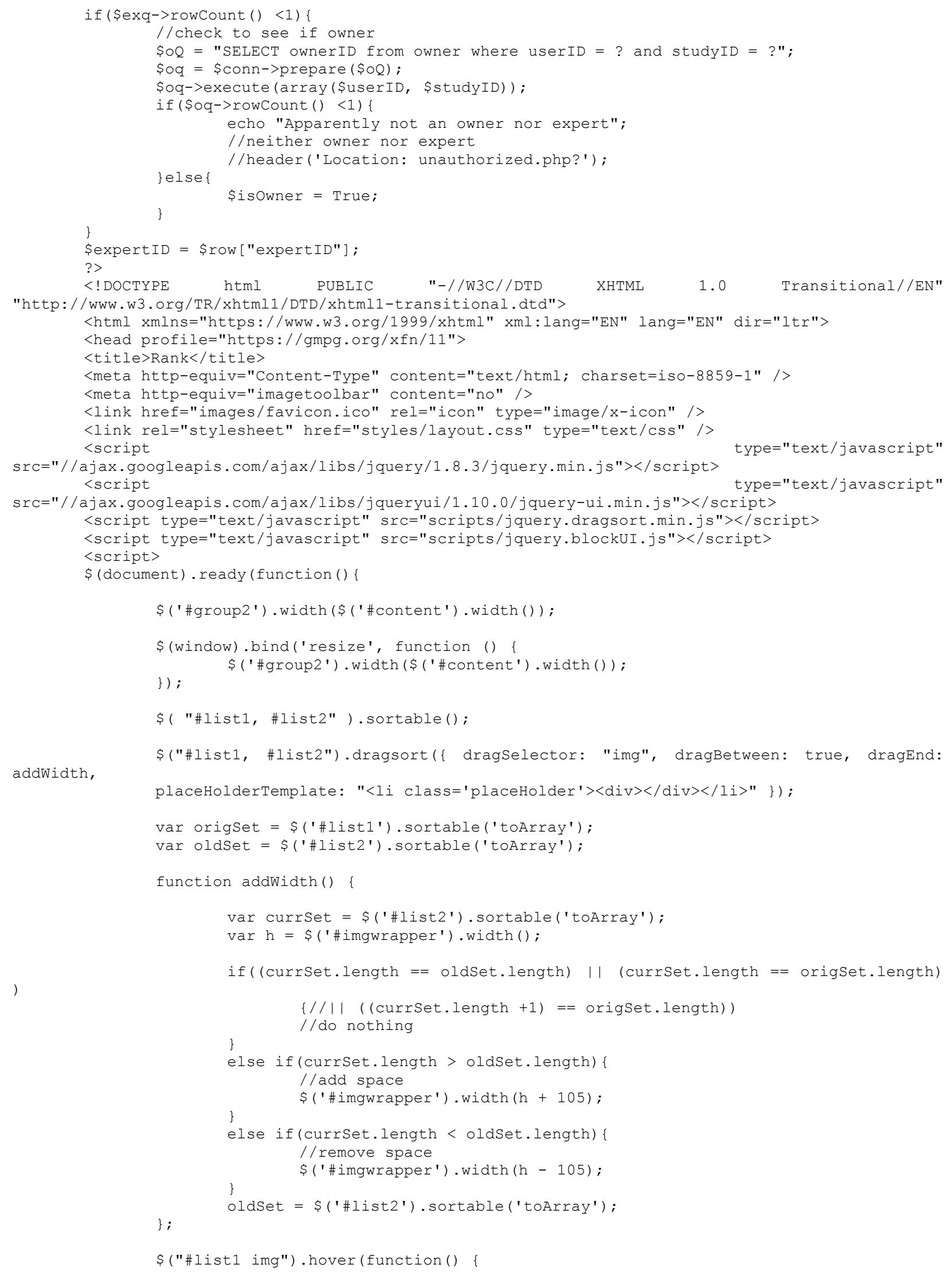




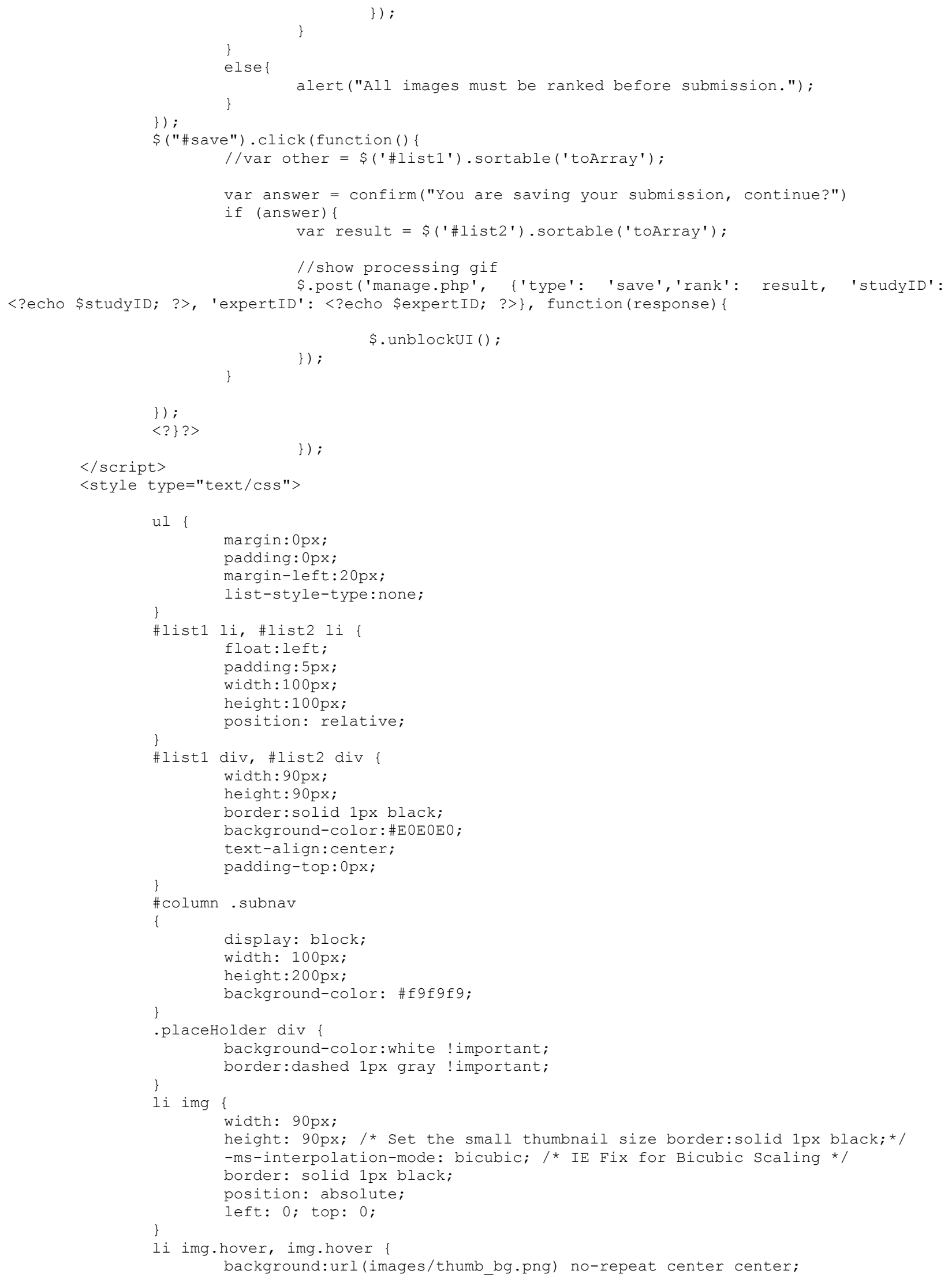




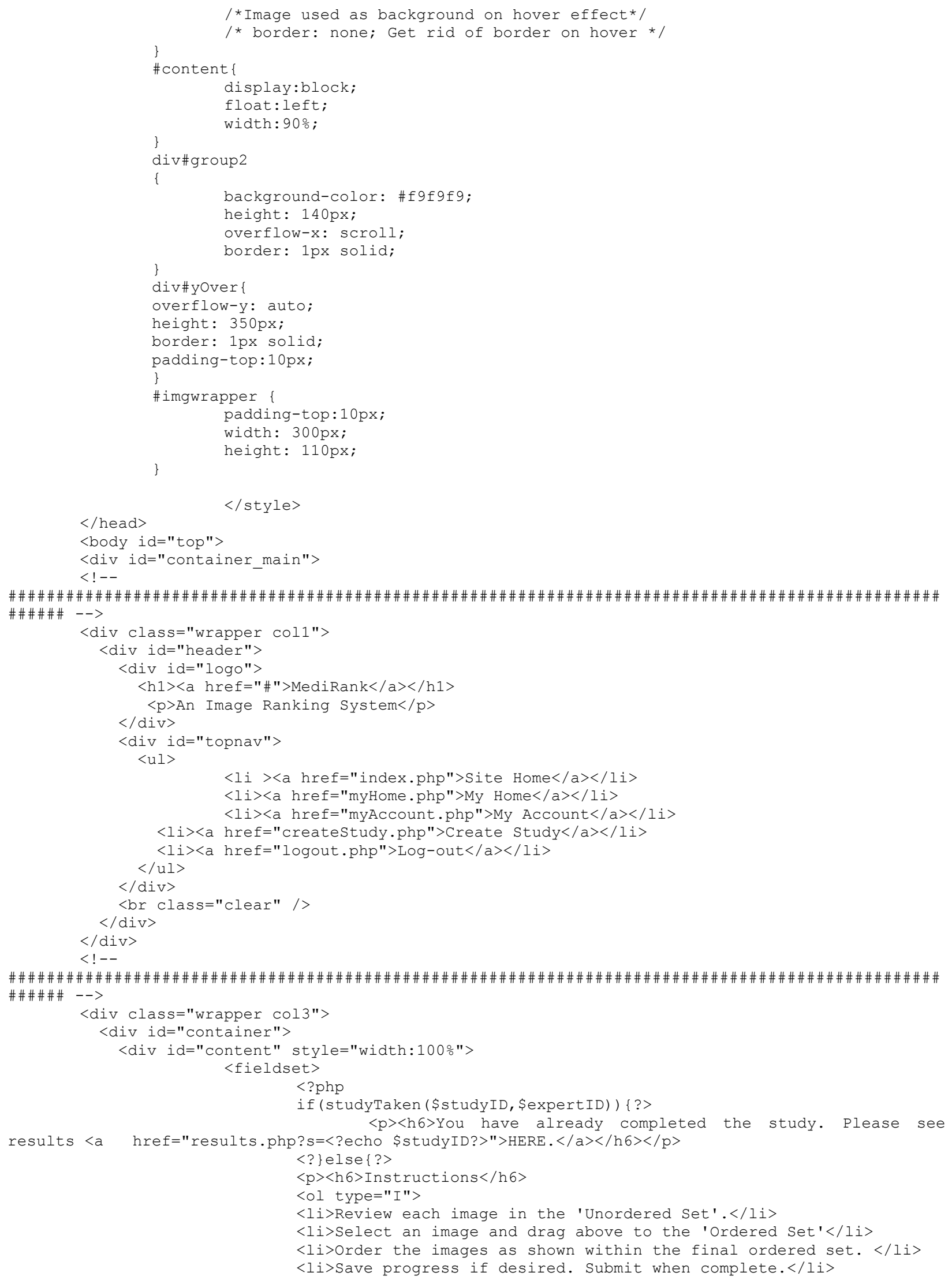




$$
</ 01></ \mathrm{p}><\mathrm{br} /><\mathrm{br} />
$$

$<$ !-- $<$ P $>$ Check this box to allow set 1 to scroll with you down the page. $<$ input type="checkbox" name="scrollBox" checked id="scrollBox" value="Enable Set 1 Scrolling" $/></ p>-->$ $<$ h6 $>$ Ordered Set $</$ h $6>$

$<\mathrm{p}><$ ?echo "Most ".\$adjective. " \&\#8594; Least " . \$adjective; ? $></ \mathrm{p}>$ $<$ div class="subnav" id="group2">

<div id="imgwrapper">

$<$ ul id="list2" >

$<$ ?

if (!studyTaken (\$studyID, \$expertID)) \{

//insert ordered images

\$saveQ

"SELECT

savePlace.placement, savePlace.imageID, images.imageName, images.imageLocation, images.studyID from savePlace join images on savePlace.imageID = images.imageID where savePlace.studyID = ? and savePlace.expertID = ? order by savePlace.placement"; $\$ \mathrm{sq}=\$ \mathrm{conn}->$ prepare $(\$ \mathrm{saveQ}) ;$ \$sq->execute (array (\$studyID, \$expertID)) ;

$\$$ num $=0$.

while (\$row $=\$$ sq->fetch ()$)\{$ echo "<li id=\" . \$row ['imageID'] "\"><img src=\"". \$row['imageLocation'] . "/" . \$studyID . "/images/" . \$row ['imageName'] . "\" alt $=\backslash "$ ". \$row ['imageName'] ." \" / $></ 1 i>"$;

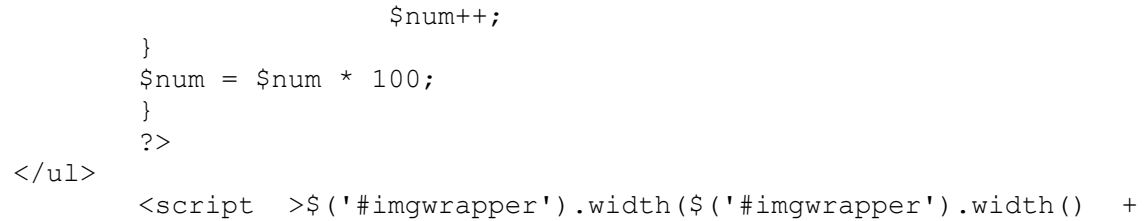




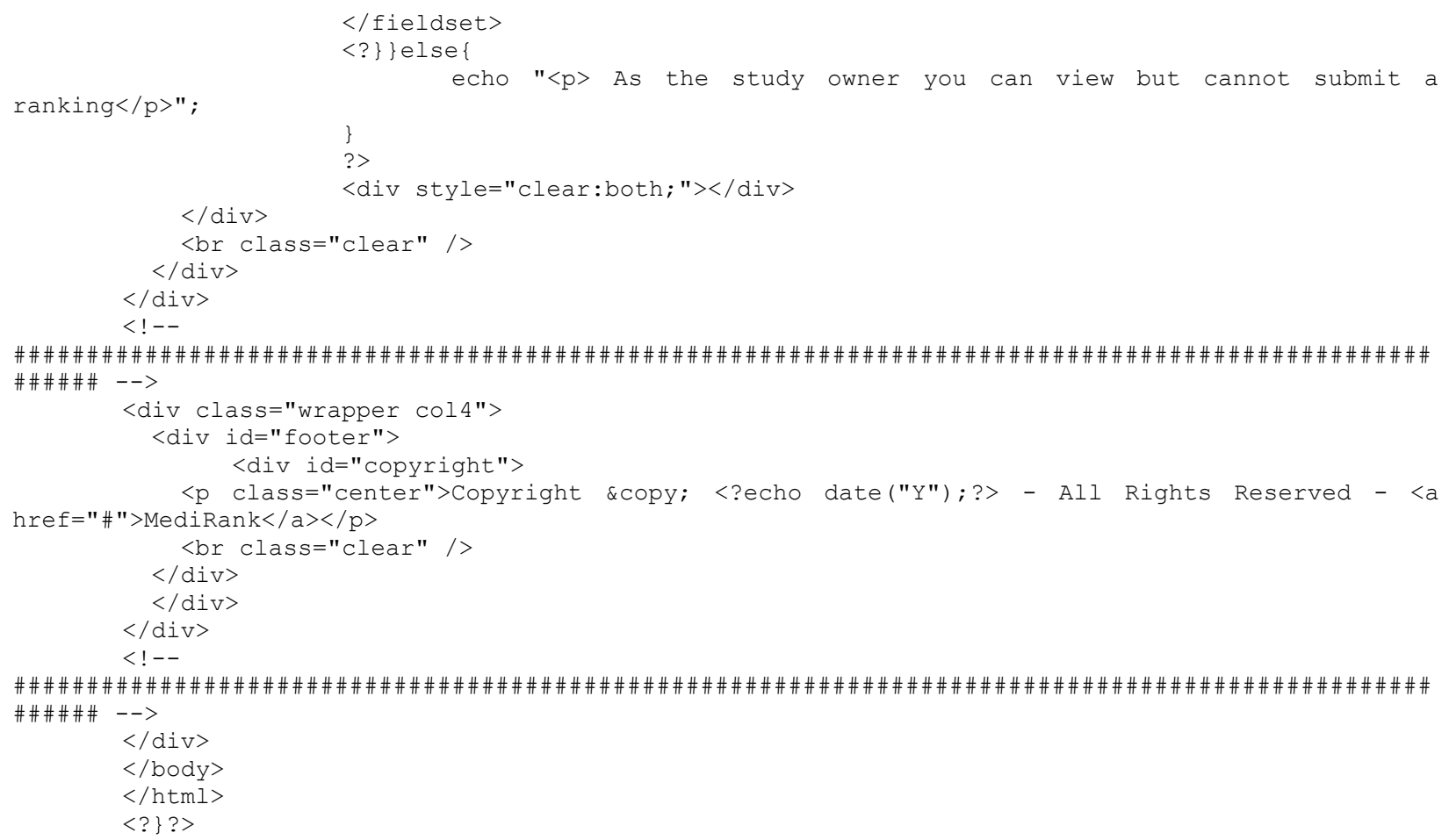

\section{D.4 results.php}

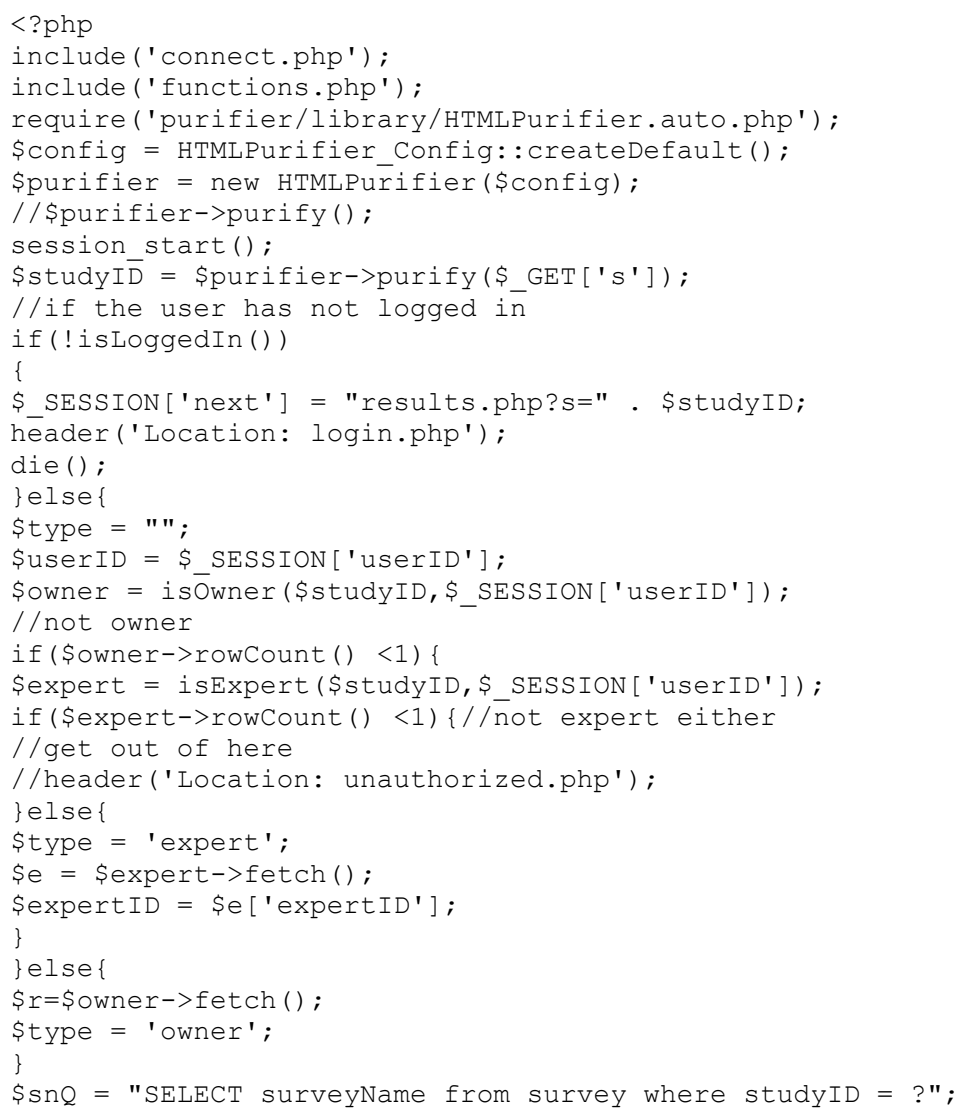




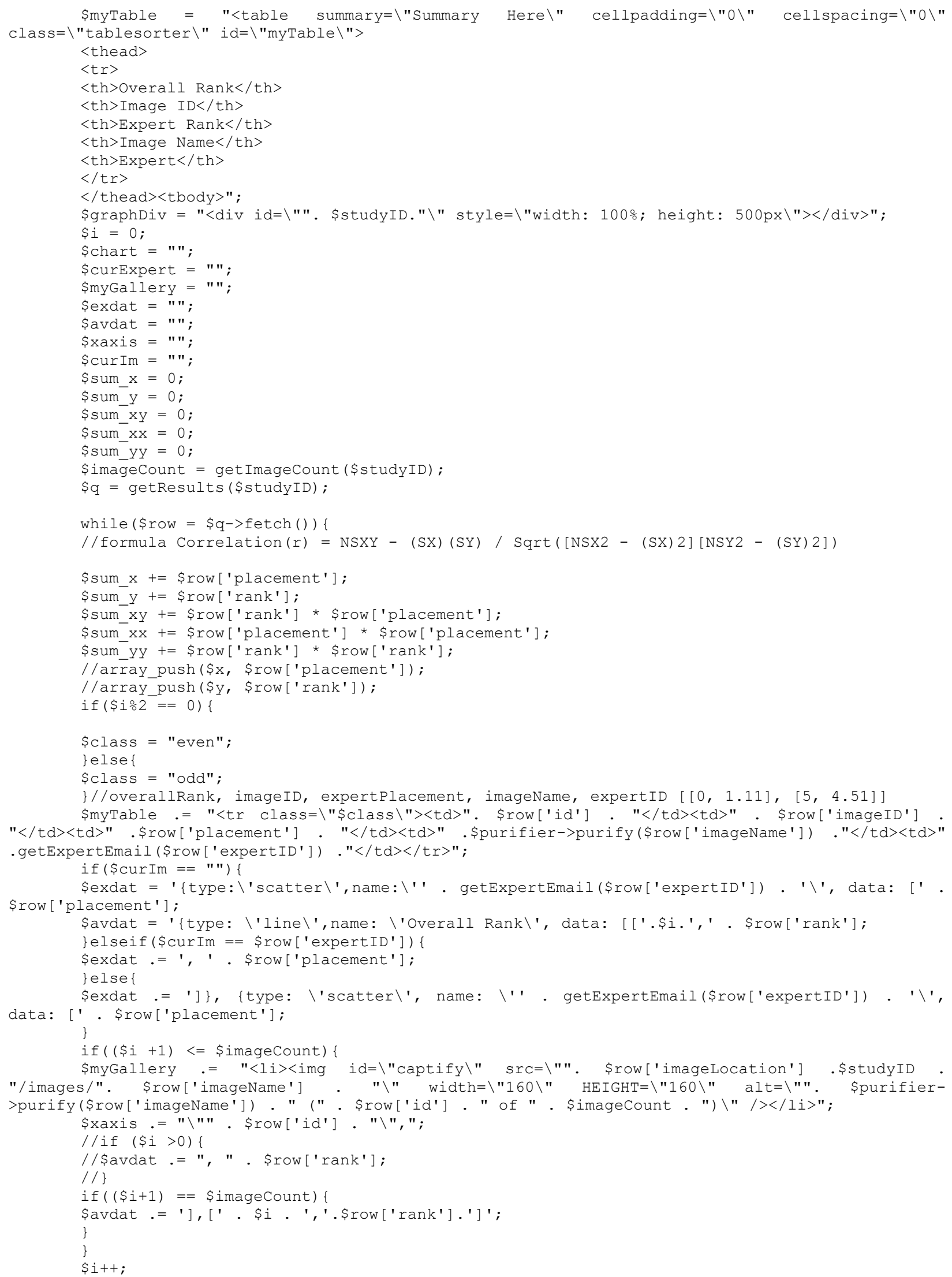




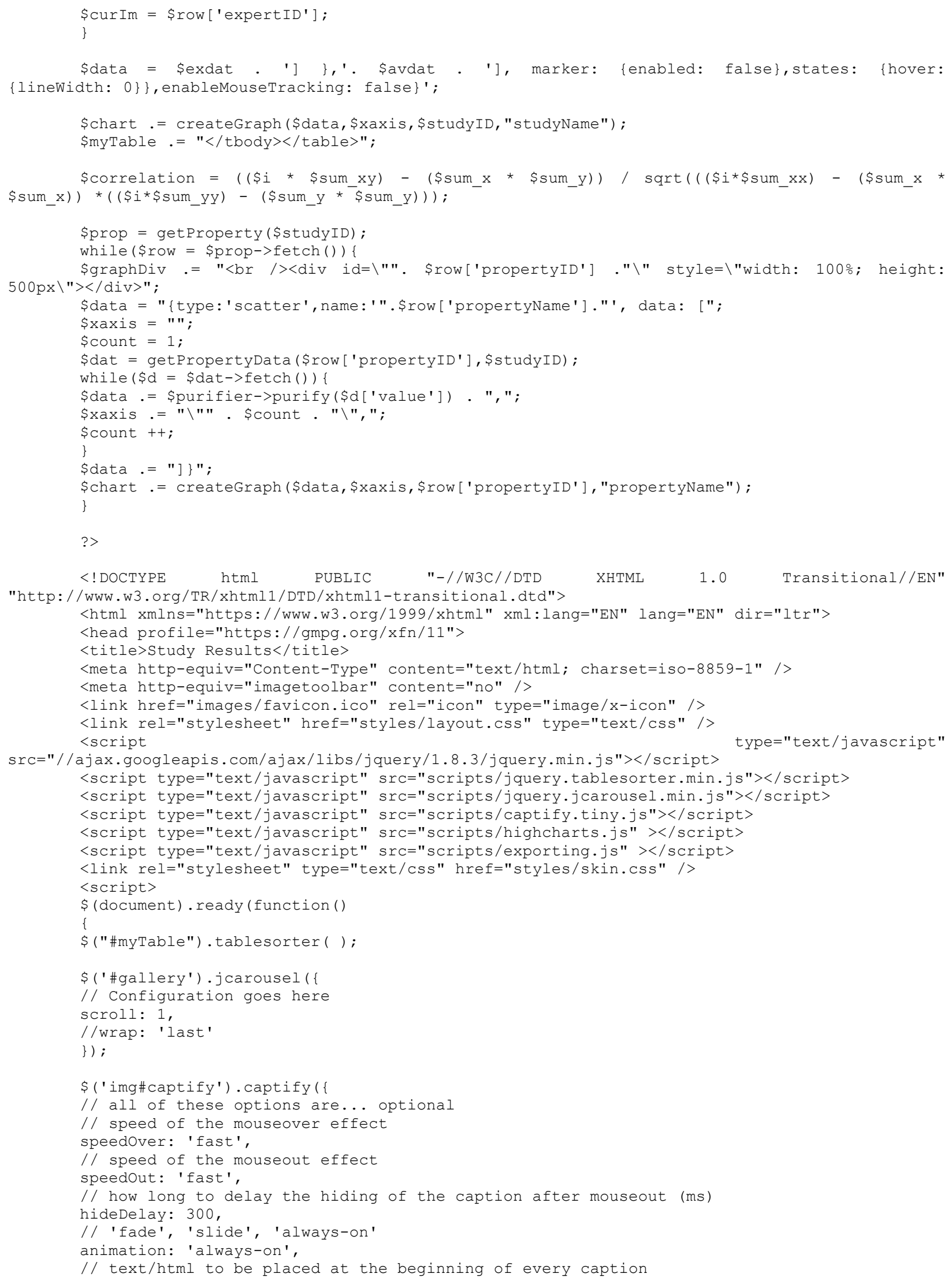




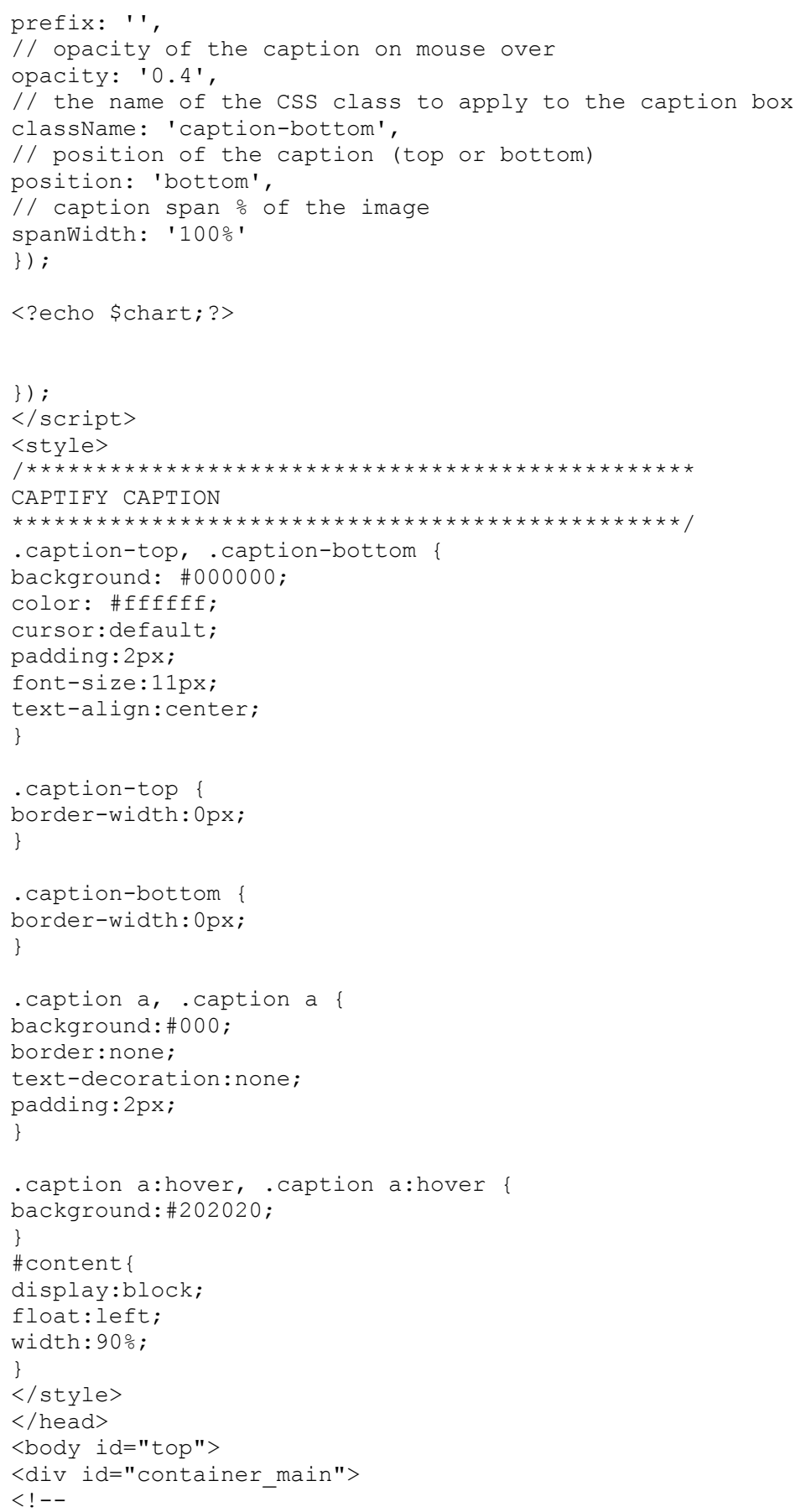




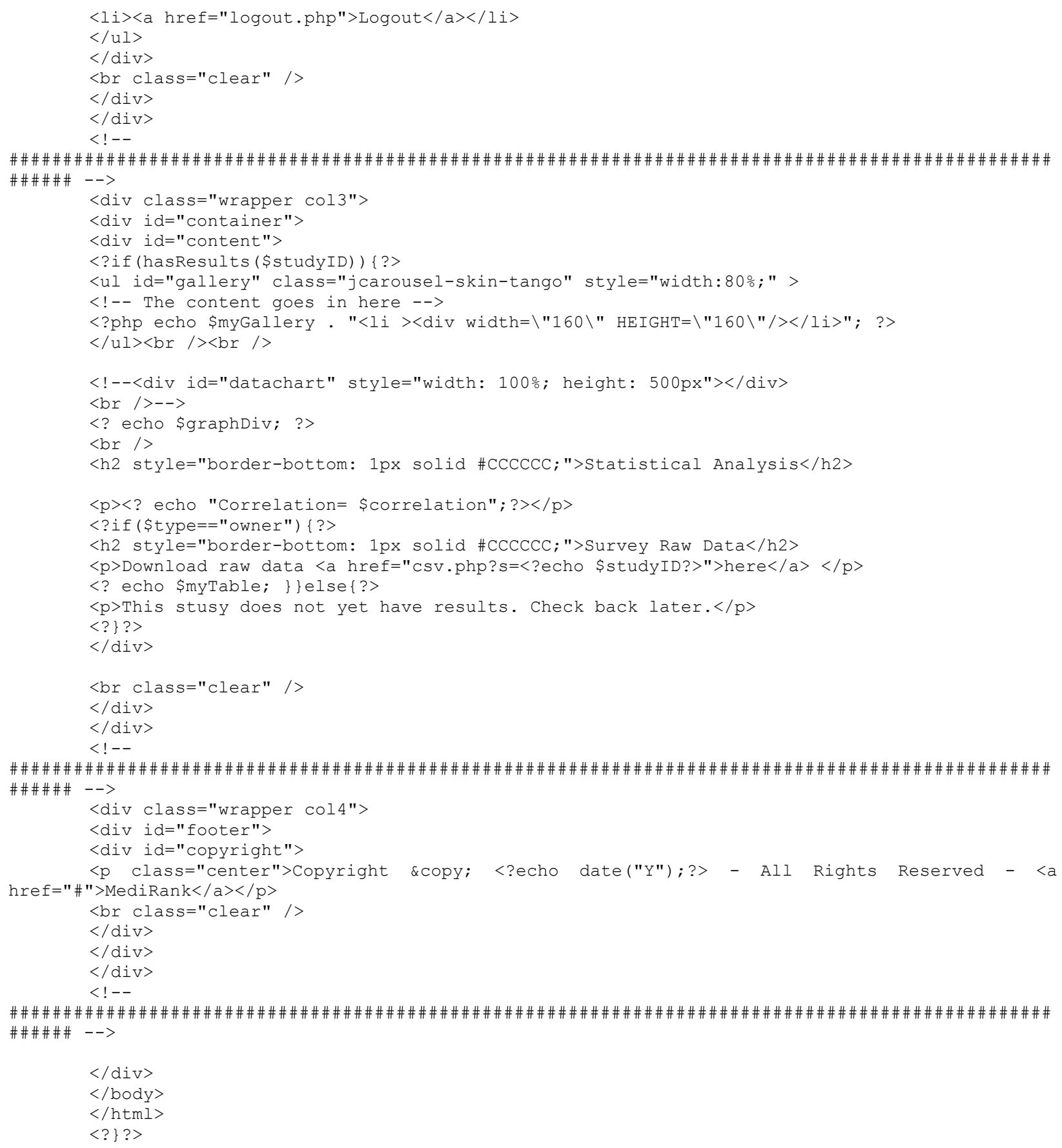

\section{D.5 manage.php}

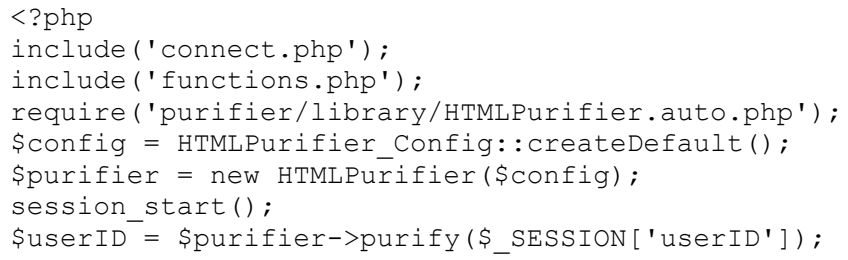




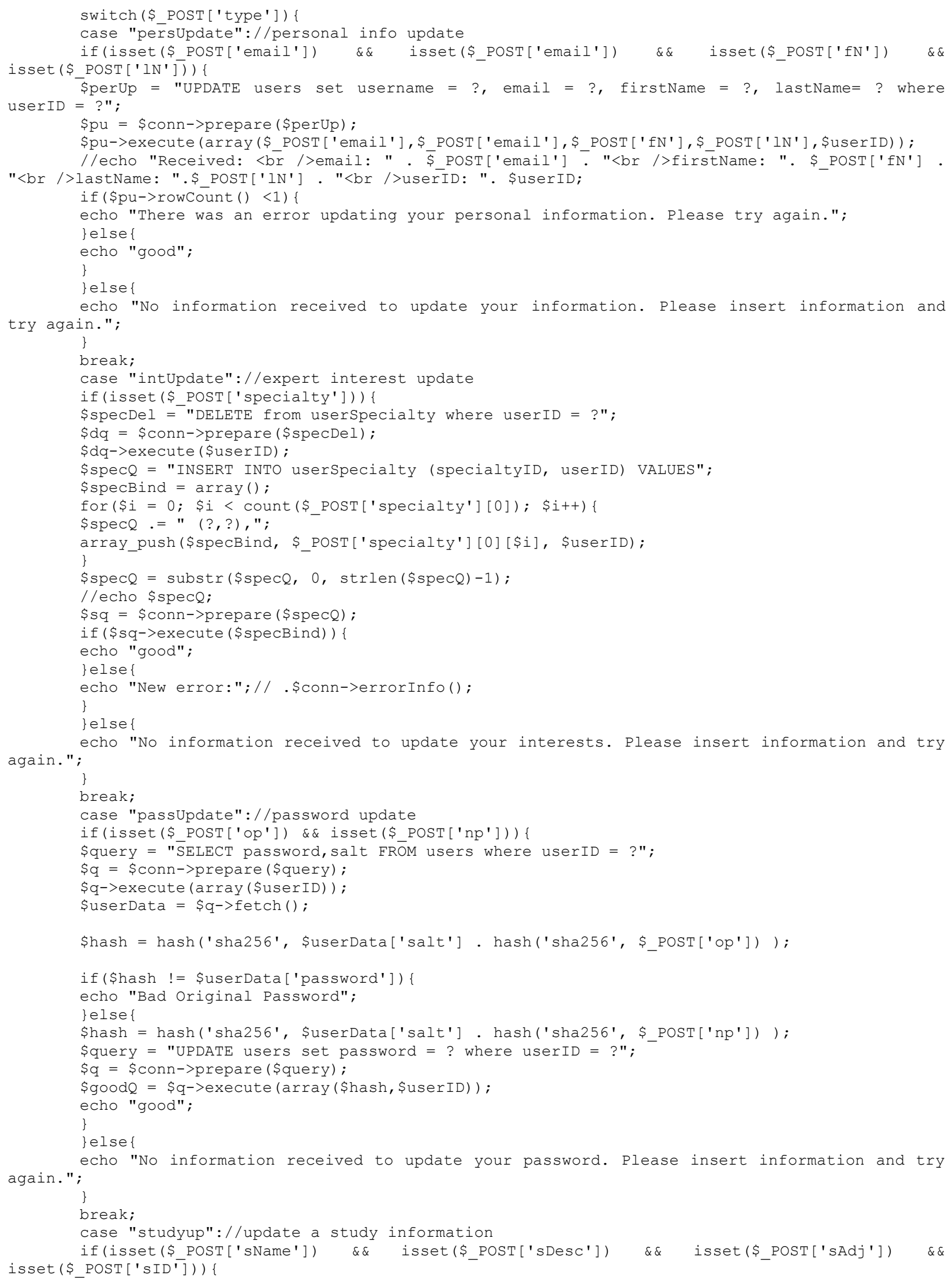


?" ;

\$SUp = "UPDATE study set studyName =?, description =?, studyAdjective=? where studyID =

\$su $=\$$ conn->prepare $(\$ \mathrm{sUp}) ;$

\$su->execute (array (\$purifier->purify (\$ POST ['sName']), \$purifier-

$>$ purify (\$_POST['sDesc']), \$purifier->purify (\$_POST['sAdj']), \$_POST['sID']));

if (\$su->rowCount () <1) \{

echo "There was an error updating your study information. Please try again. <br / ><br

/>If you did not change anything disregard this message.";

\}else \{

echo "good";

\}

\}else \{

echo "No information received to update the study. Please insert information."; \}

break;

case "expert"://add experts from study update page

if (isset (\$ POST['experts']) \&\& isset (\$POST['SID']) \&\& isset(\$ POST['O'])) \{

//Getting published status

\$published $=$ getPubstat $(\$ \operatorname{POST}['$ SID']).

foreach (\$_POST['experts'] as \$expert) \{

if((\$expert $!="$ " | \$expert $!=$ null || \$expert != " ") \&\& (filter var(\$expert,

FILTER VALIDATE EMAIL)) )

\$exArray $=$ split (", ", \$expert);

/ / var dump (\$exArray);

//checking to see if they are in the system

\$newEx = "SELECT userID from users where username = ?";

\$ne $=$ \$conn->prepare (\$newEx);

\$ne->execute (array ( \$exArray [0]));

if (\$ne->rowCount () <1) \{

//user not in the system make new user, no password

\$uID = newUser (\$exArray [0]);

\}else \{

$\$ u=\$ n e->$ fetch ()$;$

\$uID = \$u['userID'];

\}

makeExpert (\$uID, \$POST['sID']);

if (\$published) \{

if (sendExpertEmail (\$uID, \$_POST ['SID'], \$_POST['O'])) \{

echo "User: ".\$purifier->purify(\$expert)." added. And email sent. $\langle$ br />";\}

else $\{$ echo "bad";

\}else\{

echo "User: ".\$purifier->purify(\$expert)." added. <br />";

\}

\}else\{echo "Unable to add". \$purifier->purify(\$expert).". Please ensure this is a valid email address.<br/>"; $\} / / * /$

\}

if (! \$publish) \{

echo "Remember to publish the study when you are ready.";

\}else\{

echo "No information received to add experts. Please insert information if you wish to add an expert to the study.";

\}

break;

case "save"://save the progress of the expert

if (isset (\$_POST['rank']) \&\& isset(\$_POST['expertID']) \&\& isset(\$_POST['studyID'])) \{

//delete everything from previous save

deleteSave (\$_POST ['expertID'], \$_POST ['studyID']);

//insert into savePlace

//ranked images first

\$val = insertResult("savePlace", \$ POST['rank'], \$_POST['expertID'], \$ POST['studyID'], 1);

sleep (3);

echo \$val:

\}else \{

echo "No information received to save progress, please try again";

\}

break;

case "rank"://final ranking

if (isset (\$_POST['rank']) \&\& isset (\$_POST['expertID']) \&\& isset(\$_POST['studyID'])) \{

//insert into results 


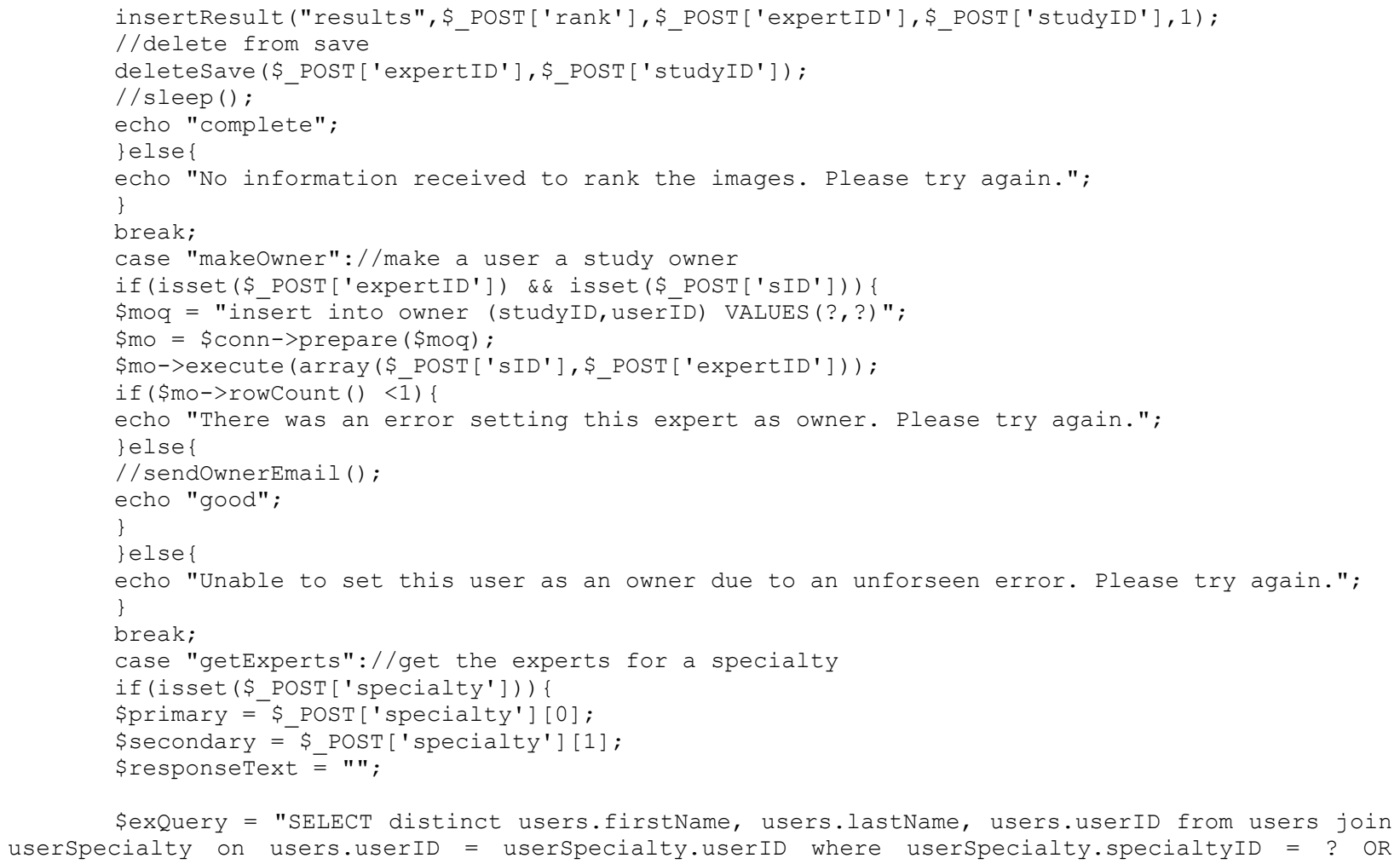




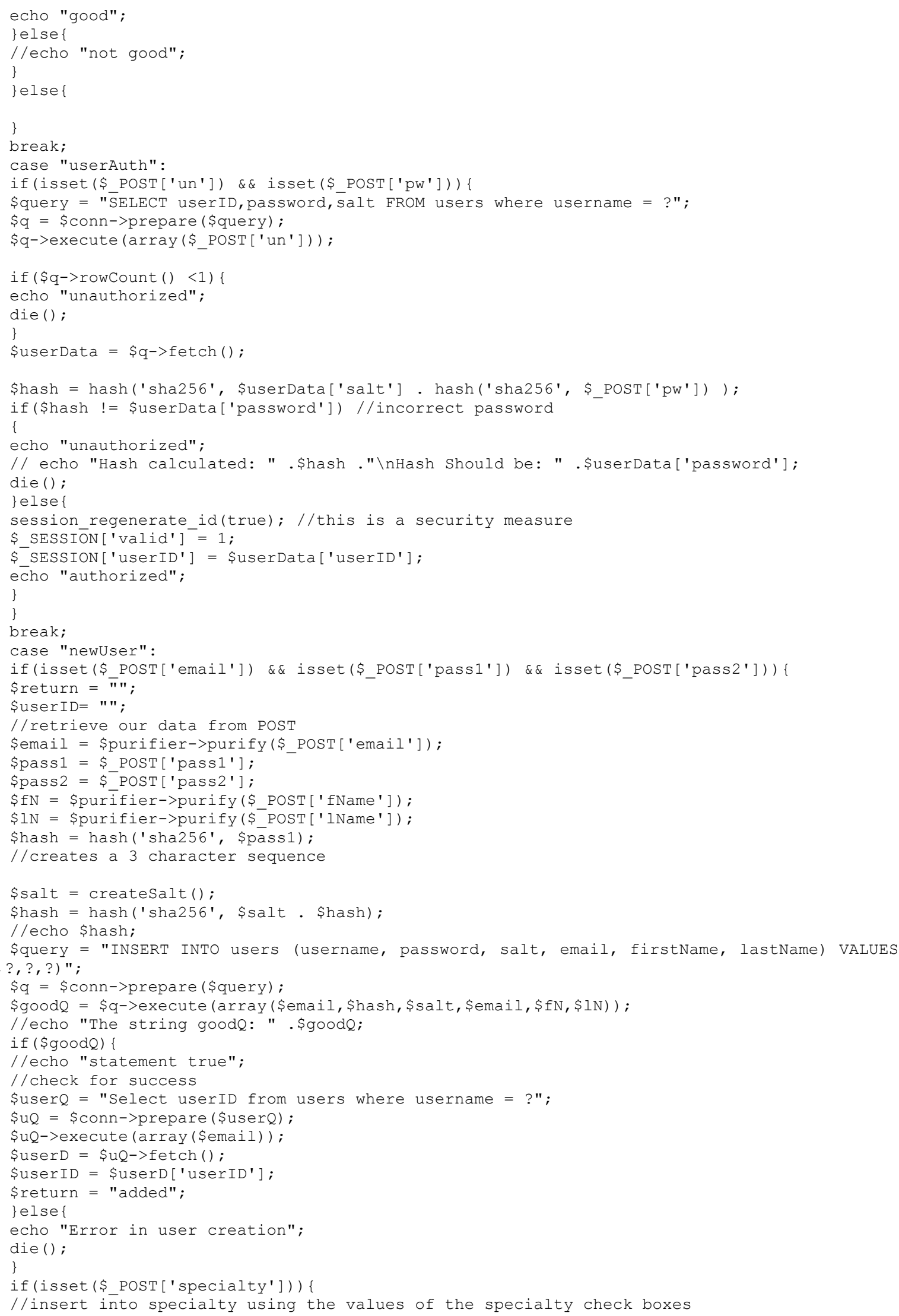




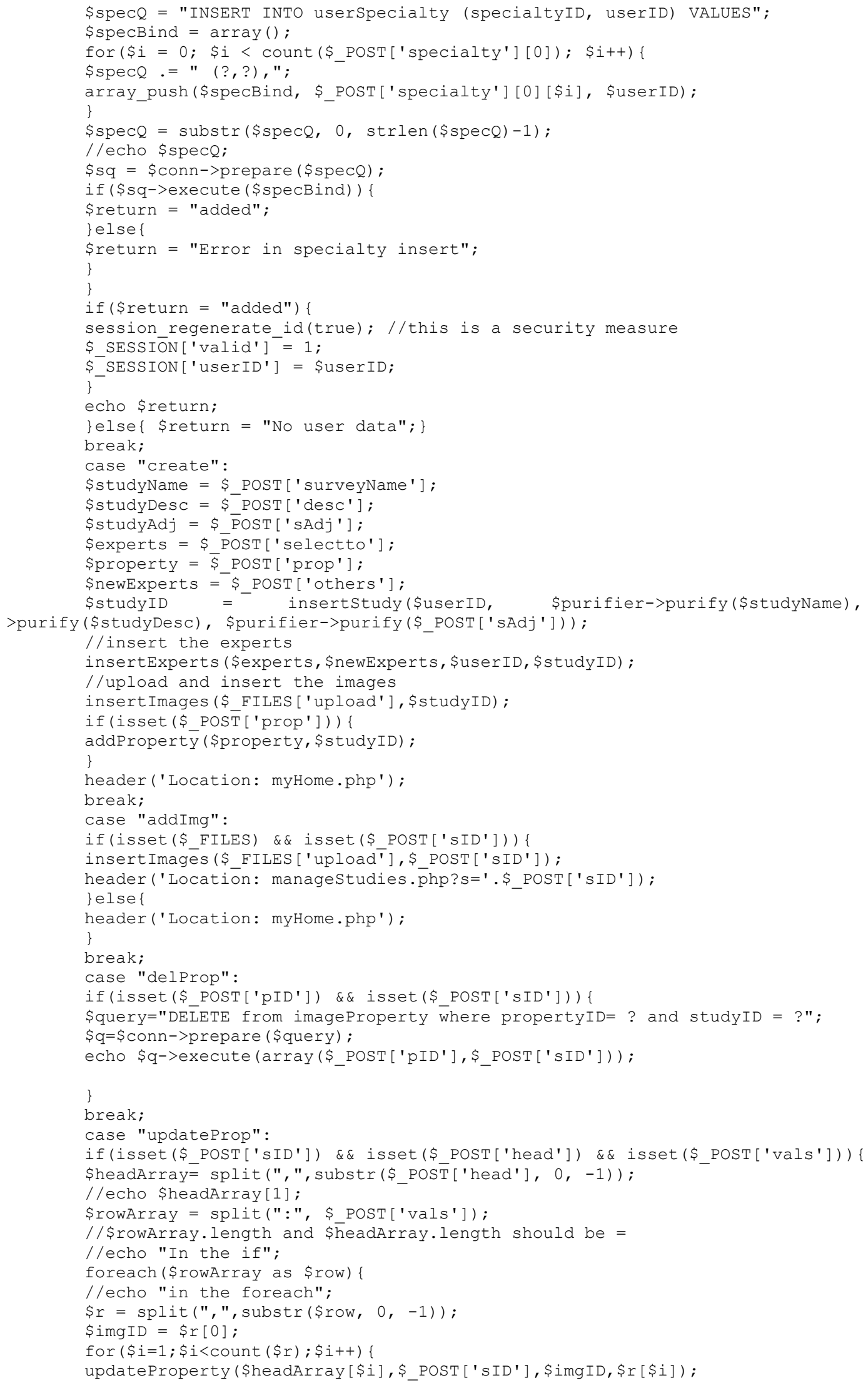




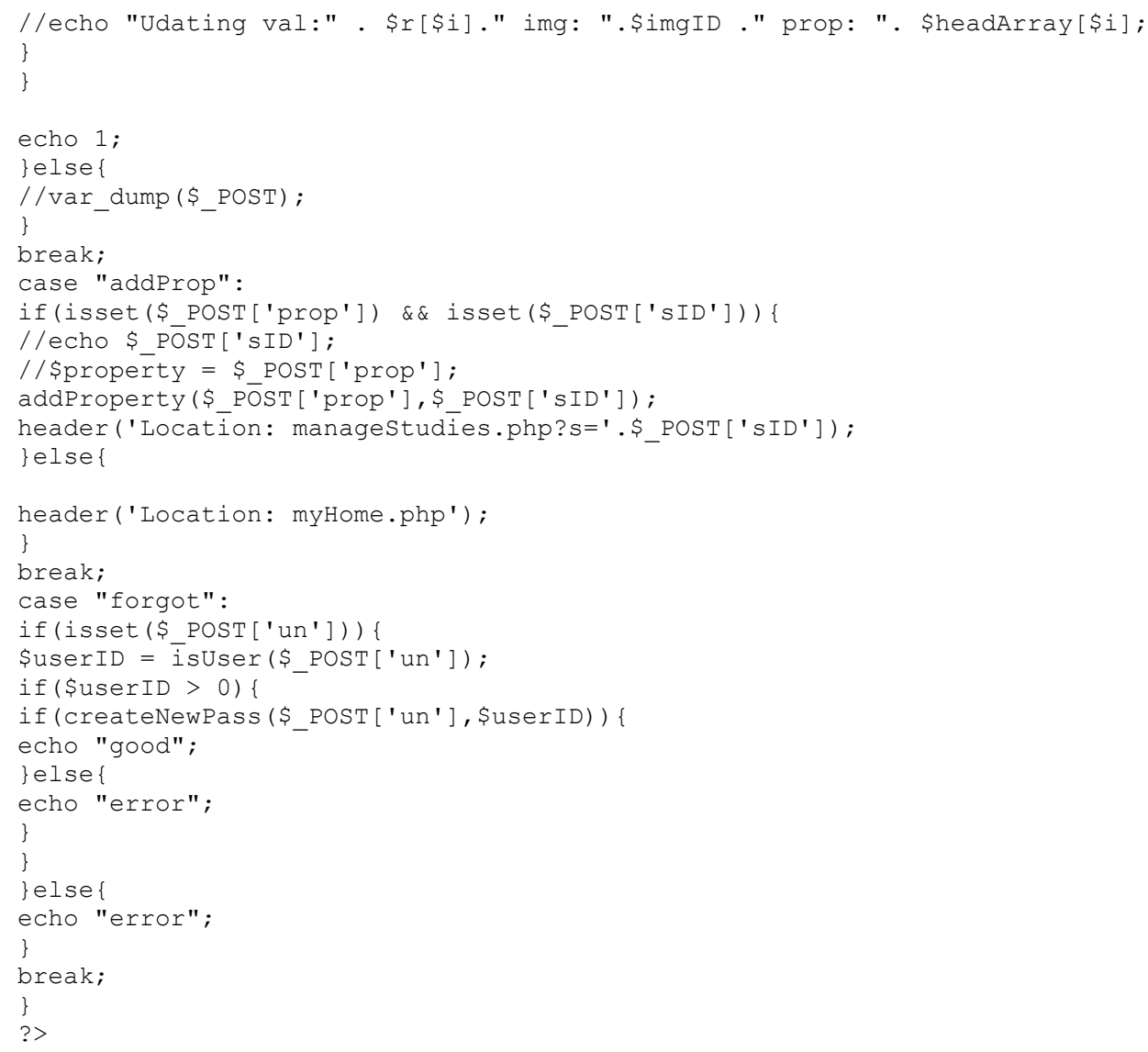

\section{D.6 functions.php}

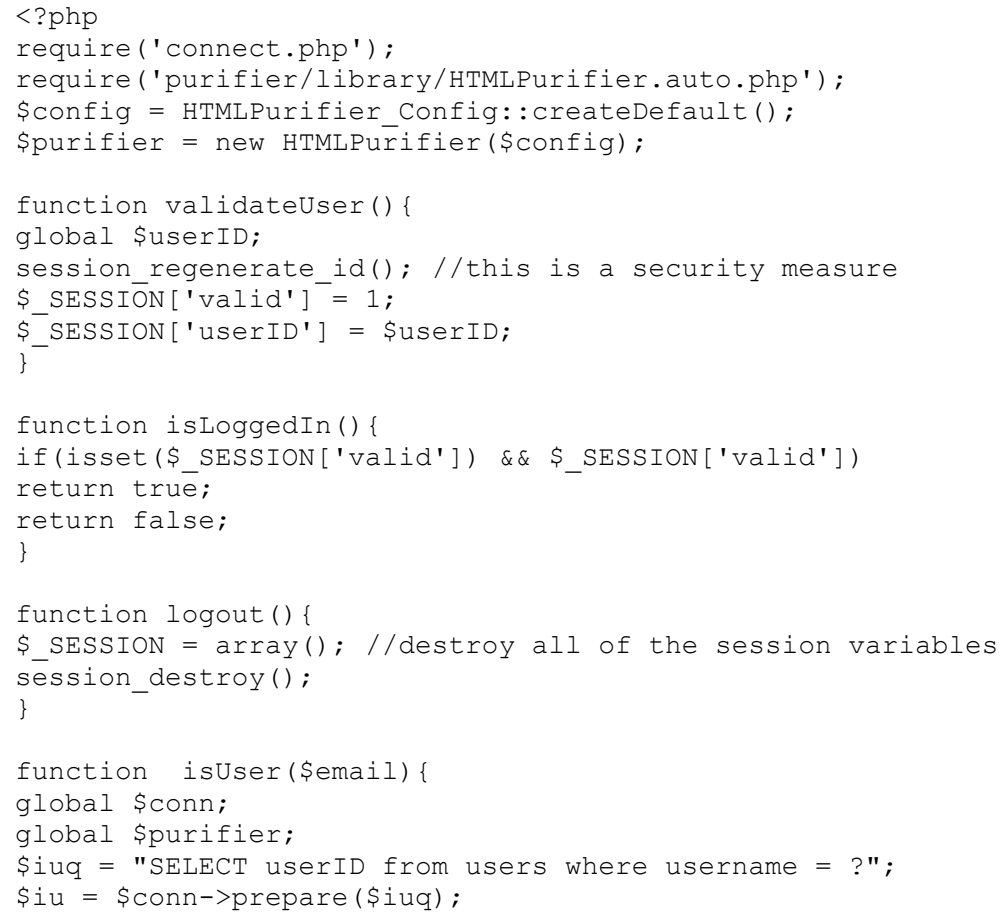


\$iu->execute (array (\$email));

\$uid = \$iu->fetch ();

return \$purifier->purify(\$uid['userID']);

\}

function createNewPass (\$email, \$uID) \{

global \$conn;

\$pass = md5 (uniqid(rand(), true));

$\$$ pass $=\operatorname{substr}(\$$ pass $, 0,8) ;$

\$hash = hash ('sha256', \$pass);

\$salt $=$ createSalt () ;

\$hash = hash ('sha256', \$salt. \$hash);

//insert new password

$\$ \mathrm{pi}=$ "update users set password $=$ ?, salt $=$ ? where userID = ?";

\$piq = \$conn->prepare $(\$ p i) ;$

if (\$piq->execute (array (\$hash, \$salt, \$uID)) ) \{

\$subject $=$ "Password reset for medirank.";

$\$$ body="As you requested, your password has now been reset. Your new details are as

follows: $\backslash n \backslash n$ Password: ". \$pass." \n\nIf you did not make this request, please change your password

at https://medirank.it.et.byu.edu/myAccount.php\n\nAll the best, \nThe Medirank Team";

\$headers = "From: Medirank<no-reply@medirank.it.et.byu.edu> $\backslash r \backslash n "$;

mail (\$email, \$subject, \$body, \$headers);

return true;

\}else\{

return false;

\}

\}

function getPubstat (\$studyID) \{

global \$conn;

\$pubq = "SELECT published from study where studyID=?";

$\$$ pub $=\$$ conn->prepare $(\$$ pubq $) ;$

\$pub->execute (array (\$studyID));

\$published = \$pub->fetch ();

return \$published['published'];

\}

function makeExpert (\$UID, \$SID) \{

global \$conn:

//add in search if they are already an expert for the study

\$isq = "SELECT * from experts where userID= ? and studyID= ?";

\$is $=\$$ conn->prepare ( $\$$ isq);

\$is->execute (array (\$UID, \$SID));

if (\$is->rowCount () <1) \{

\$meq = "INSERT into experts (userID, studyID) VALUES(?,?)";

\$me $=$ \$conn->prepare (\$meq);

//echo "inside make expert in if";

return \$me->execute(array(\$UID, \$sID));

\}else\{

echo "user already an expert";

return true;

\}

function newUser(\$un) \{

global \$conn;

global \$purifier;

\$un = \$purifier->purify (\$un);

\$nuq = "INSERT INTO users (username, email) VALUES(?,?)";

$\$$ nu $=\$$ conn->prepare (\$nuq);

\$nu->execute (array (\$un, \$un)),

\$nuq2 = "SELECT userID FROM users WHERE username = ?";

$\$$ nu2 $=$ \$conn->prepare $($ \$nuq2 $) ;$

\$nu2->execute (array (\$un));

\$id $=\$$ nu2->fetch () ;

return \$id['userID'];

\}

//Expert userID, studyID, ownerID, in system

function sendExpertEmail (\$UID, \$SID, \$sender) \{ 
global \$conn;

\$emq = "SELECT email, firstName, lastName, password from users where userID = ?";

\$em $=$ \$conn->prepare $($ Semq);

\$study $=$ getstudyInfo(\$sID);

if (\$em->execute (array (\$uID)) ) \{

\$info $=$ \$em->fetch ():

\$to $=$ \$info['email'];

\$subject $=$ "Medirank study participation";

\$headers = "From: Medirank<no-reply@medirank.it.et.byu.edu> \r\n";

\$oemg = "SELECT firstName, lastName FROM users WHERE userID = ?";

\$oem $=$ \$conn->prepare $(\$ o e m q) ;$

\$oem->execute (array (\$sender));

\$oi $=$ \$oem->fetch ()$;$

if (\$info['password'] != "") \{

\$body = \$info['firstName']. " " . \$info['lastName'] . ", \n". \$oi['firstName'] ." " .

\$oi['lastName']." requests your participation in an image ranking study. $\backslash n \backslash n$

The title of the study is ". \$study['studyName']. " and a description is ".

\$study ['description']." \n\n

To complete this study please go to http://medirank.it.et.byu.edu/rank.php?s=\$sID. \nWe appreciate your participation. You can see partial results of this study at

http://medirank.it.et.byu.edu/results.php?s=\$sID.\nAgain, thank-you for your participation.";

if $(\$ S I D==2253)\{$

$\$$ body .= "\n\nPlease be sure to take a quick survey here when you are done:

https://qtrial.qualtrics.com/SE/?SID=SV ahsqge4ckuPMcsJ $\backslash$ After completing the ranking you should be redirected to that survey. If not contact me and I'll send you the link. $\backslash n \backslash n I$ really appreciate your help. \n\nThanks again\nTodd";

\}

\}elseif (\$info['password'] == " ") \{

//make random password

\$pass $=$ md5 (uniqid (rand (), true))

\$pass $=$ substr $(\$$ pass, 0,8$) ;$

\$hash = hash ('sha256', \$pass);

$\$$ salt $=$ createsalt ()

\$hash $=$ hash ('sha256', \$salt. \$hash);

//insert new password

$\$ \mathrm{pi}=$ "update users set password $=$ ?, salt = ? where userID = ?";

\$piq $=$ \$conn->prepare (\$pi);

if (\$piq->execute (array (\$hash, \$salt, \$uID)) ) \{

\$body = "\n". \$oi['firstName']." ". \$oi['lastName']." requests your participation in an image ranking study by. \n\nThe title of the study is " . \$study['studyName'] . " and a description is ". \$study['description']."\n\nTo complete this study please go to

https://medirank.it.et.byu.edu/rank.php?s=\$sID. \n Your log-in information is below. \n\nusername: \$to \npassword: \$pass $\backslash n \backslash n W e$ appreciate your participation. You can see partial results of this study at http:/medirank.it.et.byu.edu/results.php?s=\$sID $\backslash$ n $\backslash$ nUpon completion of the study please take a moment and update your information including your password at

http://medirank.it.et.byu.edu/myAccount.php. \n\nAgain, thank-you for your participation."; if $(\$ S I D==1156)$

\$body .= "\n\nPlease be sure to take a quick survey here when you are done:

https://qtrial.qualtrics.com/SE/?SID=SV_3rVsEgubNNOL1R3\nAfter completing the ranking you should

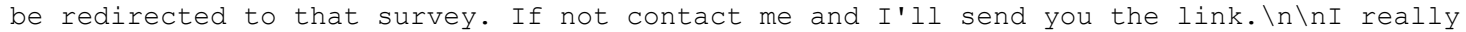

appreciate your help. I will be defending around the 10 th of December. $\backslash n \backslash n T h a n k s$ again $\backslash$ nTodd";

\}else $\{$ return false;

\}

mail (\$to, \$subject, \$body, \$headers);

return true;

\}else \{

return false;

function setPubstat (\$SID)

global \$conn;

\$query = "UPDATE study set published=1 where studyID=?";

$\$ q=\$ c o n n->$ prepare (\$query);

\$q->execute (array (\$sID));

return true 
function getstudyInfo(\$SID) \{

global \$conn;

\$query = "SELECT study.studyName, study.description, study.studyAdjective from study where studyID=?" ;

$\$ q=\$ c o n n->$ prepare $(\$ q u e r y) ;$

\$q->execute (array (\$sID)) ;

return $\$ q->$ fetch () ;

\}

function insertResult (\$loc, \$results, \$expertID, \$studyID, \$p) \{

global \$conn;

\$length $=$ count $(\$ r e s u l t s) ;$

if $(\$ l O C==$ "results" || $\$ l O C==$ "savePlace") \{

for $(\$ i=0 ; \$ i<\operatorname{count}(\$ r e s u l t s) ; \quad \$ i++)\{$

if $(\$ \mathrm{p}==0)\{$ placement $=0 ;\}$ else $\{$ placement $=\$ i+1 ;\}$

\$imageID = \$results[\$i];

\$rQuery = "INSERT INTO \$lOC (studyID, placement, imageID, expertID) VALUES (?,?,?,?) ON

DUPLICATE KEY UPDATE placement = ?";

$\$ q=\$ c o n n->$ prepare (\$rquery);

\$rval = \$q->execute (array (\$studyID, \$placement, \$imageID, \$expertID, \$placement));

\}

\}else \{

\$rval = 0;

\}

return \$rval

\}

function deleteSave (\$eID, \$sID) \{

global \$conn;

\$saveDel = "DELETE from savePlace where expertID = ? and studyID = ?";

$\$ \mathrm{sDel}=\$ \mathrm{conn}->$ prepare $(\$ \mathrm{saveDel}) ;$

return \$sDel->execute (array (\$eID, \$sID));

\}

function studyTaken (\$studyID, \$expertID) \{

global \$conn;

\$query = "select * from results where studyID = ? and expertID = ?";

\$st $=$ \$conn->prepare (\$query);

\$st->execute (array (\$studyID, \$expertID));

if (\$st->rowCount () <1) \{

return false;

\}elseif (\$st->rowCount() >=1) \{

return true;

\}

function createsalt()

\$string $=\operatorname{md5}($ uniqid(rand(), true)) ;

return substr(\$string, 0, 3);

\}

function isOwner(\$sID, \$UID) \{

global \$conn;

\$ownQ = "SELECT ownerID from owner where userID = ? and studyID = ?".

\$ownq $=$ \$conn->prepare $($ SownQ $)$;

\$ownq->execute (array (\$uID, \$sID));

return \$ownq;

function isExpert (\$SID, \$uID) \{

global \$conn;

\$expQ = "SELECT expertID from experts where userID = ? and studyID = ?";

\$exq $=\$ c o n n->$ prepare $(\$ \operatorname{expQ}) ;$

\$exq->execute (array (\$uID, \$sID));

return \$exq

\} 
function getImageCount (\$SID) \{

global \$conn;

\$imageQuery = "SELECT count ('imageID') as count FROM images WHERE studyID = ?";

$\$ q=\$ c o n n->$ prepare (\$imageQuery);

$\$ q->$ execute (array (\$sID)) ;

\$row $=\$ q->$ fetch ()$;$

return \$row ["count"];

\}

function getResults (\$studyID) \{

global \$conn;

\$tempQuery ="create temporary table tmptbl (id INT UNSIGNED NOT NULL PRIMARY KEY AUTO_INCREMENT, imageID varchar (10), rank decimal $(14,4)$, imageName varchar(100), imagelocation varchar (200))";

$\$ q=\$ c o n n->$ prepare (\$tempQuery);

\$q->execute () ;

\$tempInsert $=$ "insert into tmptbl (imageID, rank, imageName, imageLocation) select

results.imageID, avg (results.placement) as rank, images.imageName, images.imagelocation from results right join images on results.imageID = images.imageID where results.studyID = ? group by

results.imageID order by rank";

$\$ q=\$ c o n n->$ prepare (\$tempInsert);

\$q->execute (array (\$studyID)) ;

\$dataQuery = "select

results.expertID, results.imageID, results.placement, tmptbl.id, tmptbl.rank, tmptbl.imageName, tmptbl. imagelocation from results right join tmptbl on results.imageID = tmptbl.imageID where

results.studyID $=$ ? order by results.expertID,tmptbl.id";

$\$ q=\$ c o n n->$ prepare (\$dataQuery);

\$q->execute (array (\$studyID));

return $\$ \mathrm{q}$;

\}

function getExpertEmail (\$expertID) \{

global \$conn:

global \$purifier;

SexInfoQ = "select users.username, experts.userID from experts join users on users.userID

= experts.userID where experts.expertID = ?";

\$exIq $=$ \$conn->prepare (\$exInfoQ);

\$exIq->execute (array (\$expertID));

\$info = \$exIq->fetch ();

return \$purifier->purify (\$info['username']);

\}

function createGraph (\$data, \$xaxis, \$itemID, \$type) \{

\$title = getItemName (\$itemID, \$type);

if (\$type == "propertyName") \{

\$text = \$title." Value";

\}elseif (\$type == "studyName") \{

\$text = "Expert Rank";

\}

\$chart $=$ "chart $=$ new Highcharts. Chart $(\{$

chart: \{

renderTo: '".\$itemID."',

\},

title:

text: '".\$title."'

$x:-20$ //center

\} ,

subtitle: \{

text: ' '

$x:-20$

\} ,

xAxis: \{

title:

enabled: true,

text: 'Ordered Images'

\}, 


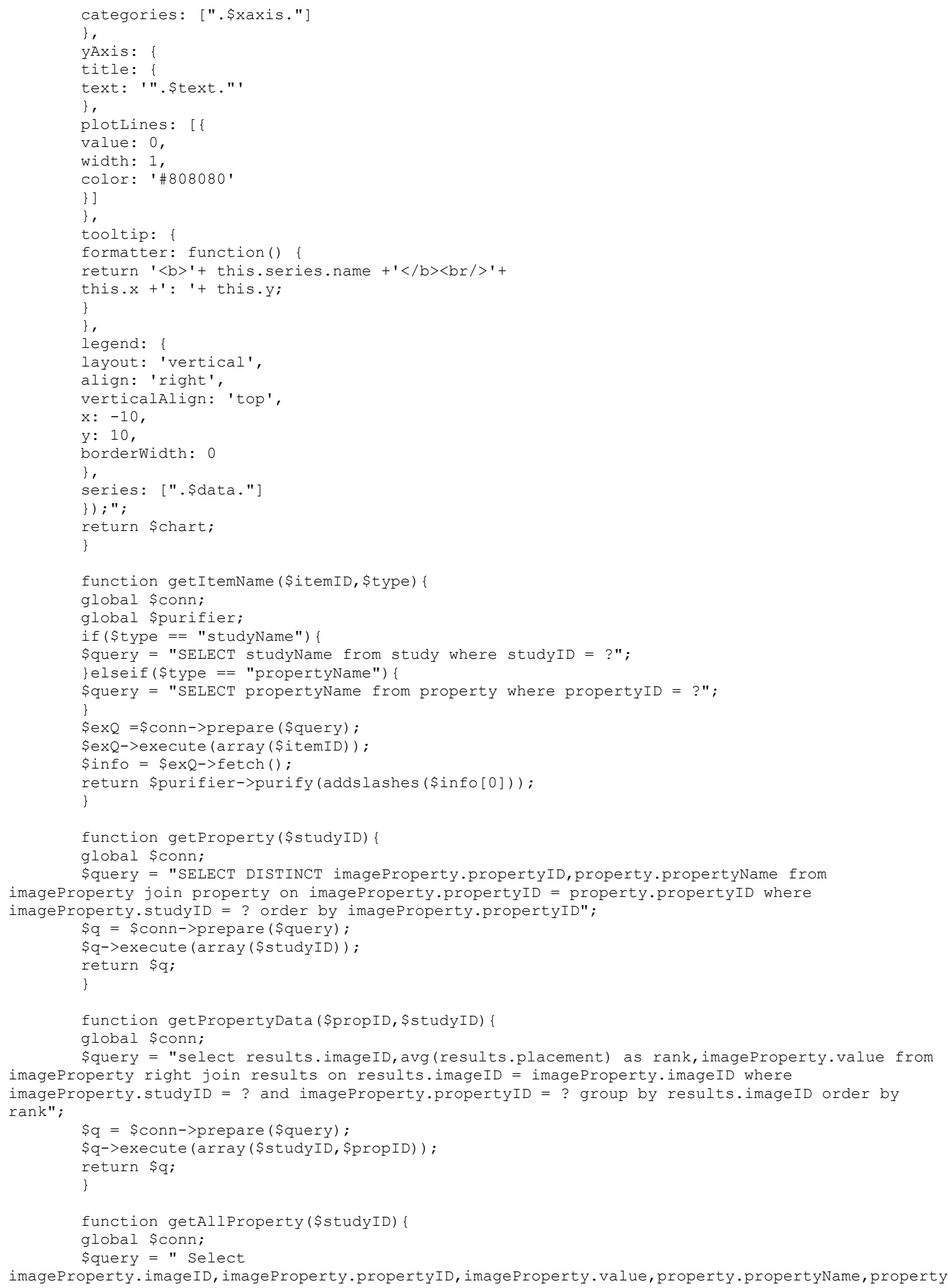




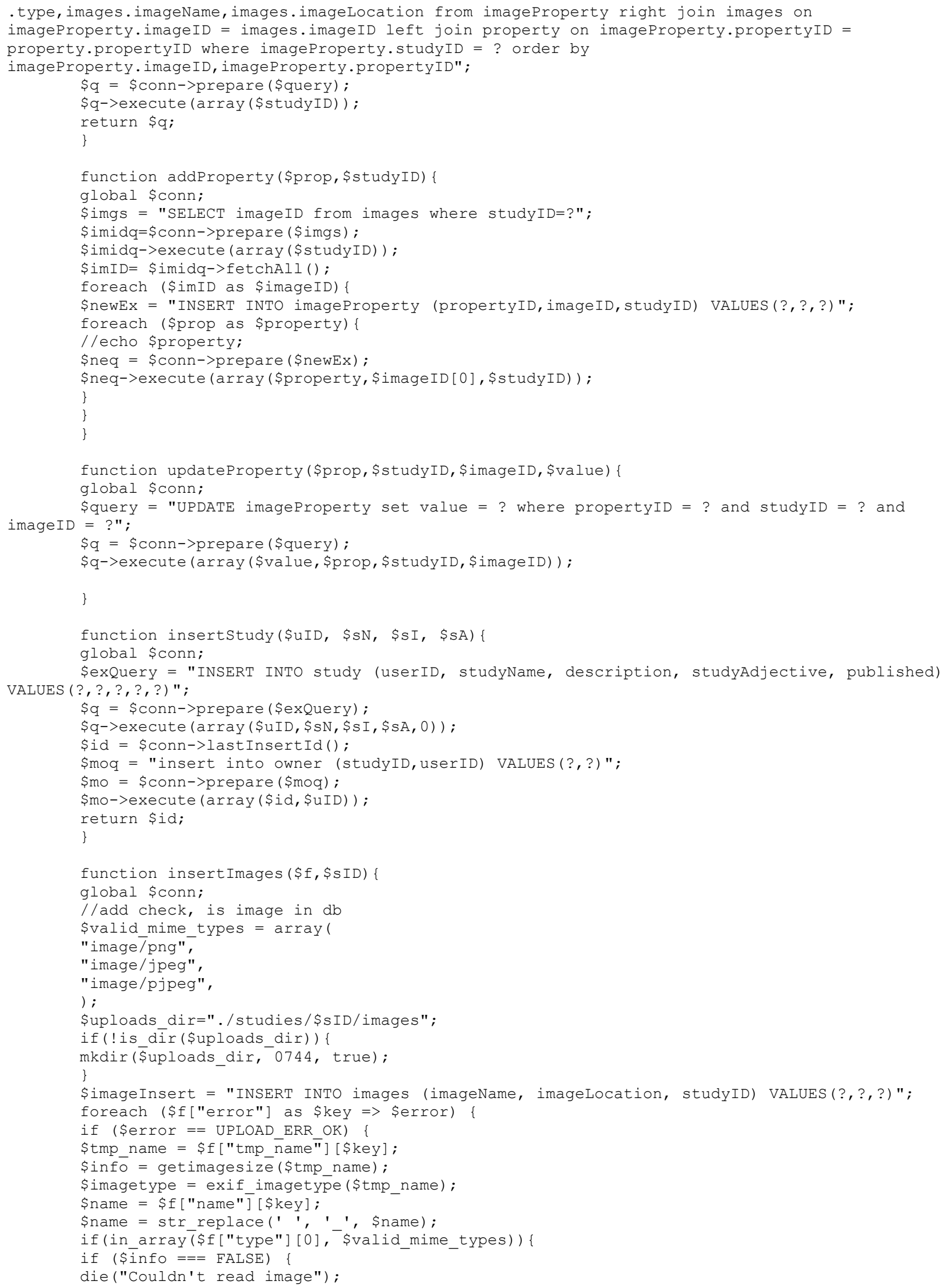




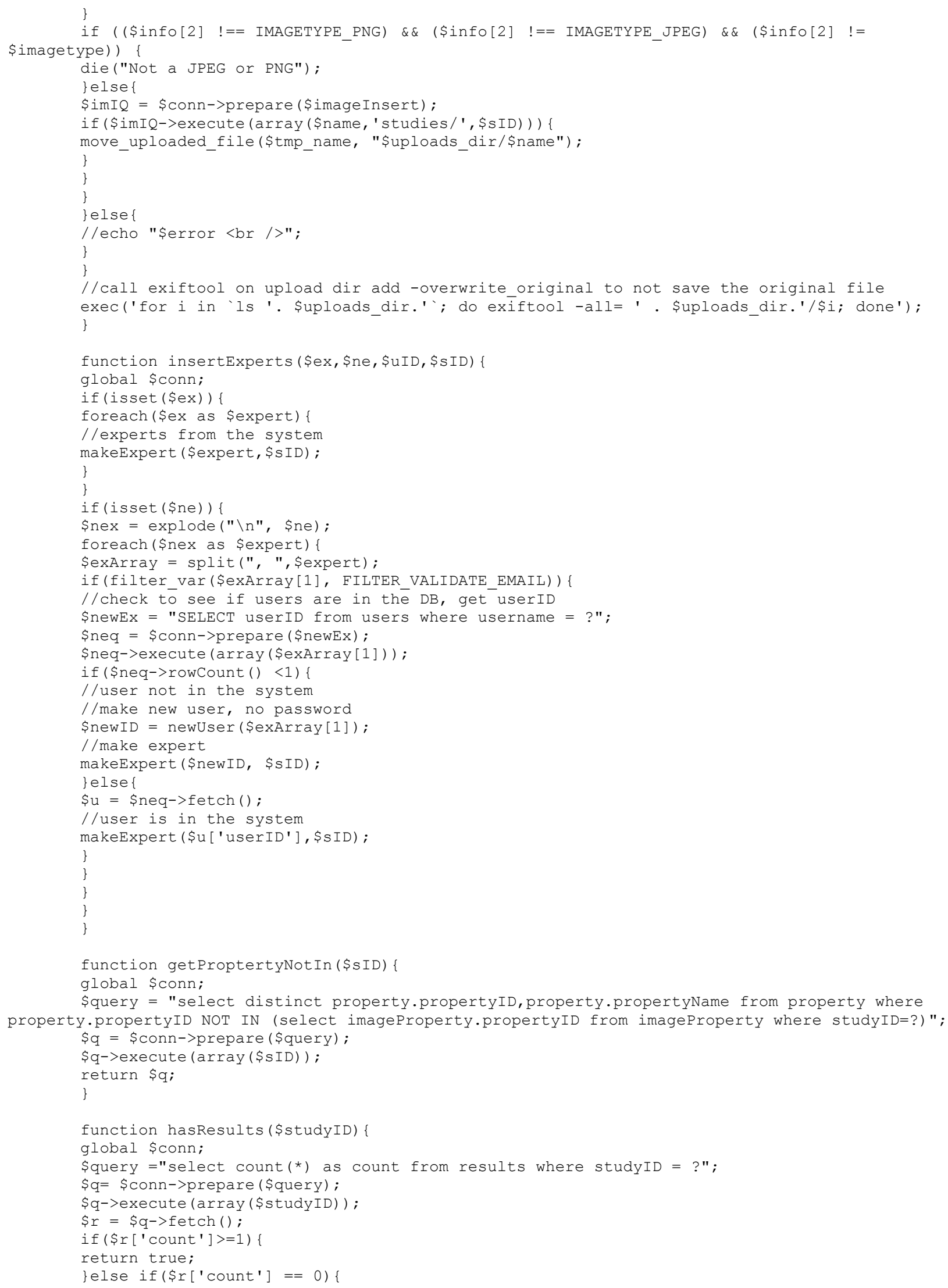




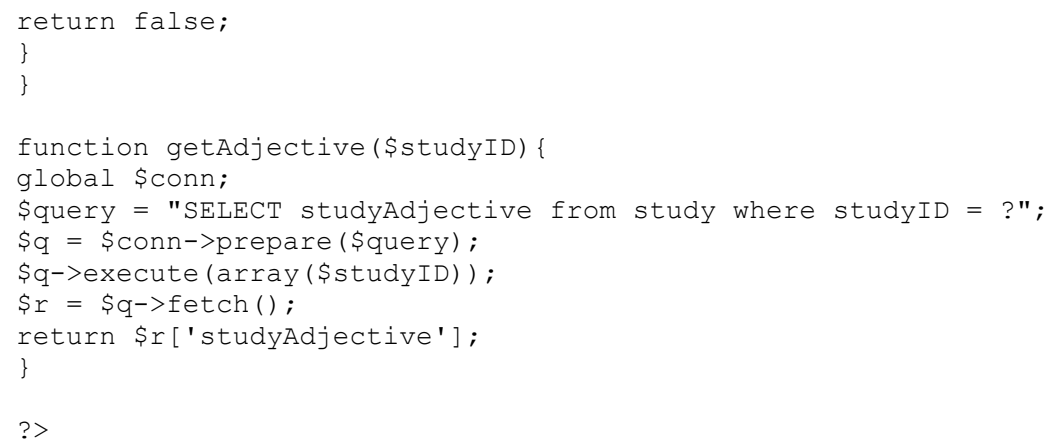

